I N T ER N ATIONAL MONETARY FUND

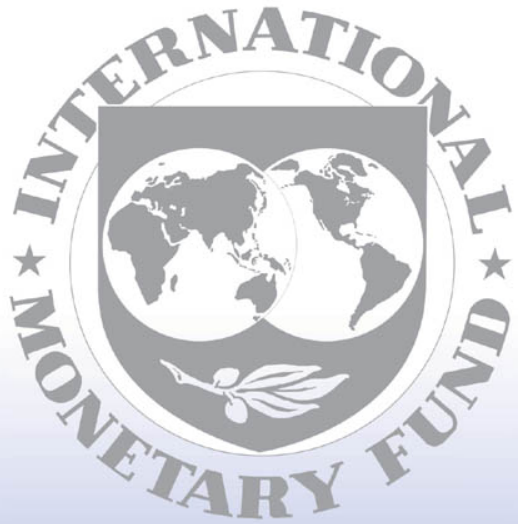

Staff

Country

Reports 


\section{Republic of Madagascar: Fourth Review Under the Poverty Reduction and Growth Facility and Requests for Extension of the Arrangement and Additional Interim Assistance Under the Enhanced Initlative for Heavily Indebted Poor Countries-Staff Report; Staff Supplement; Staff Statement; Press Release on the Executive Board Discussion; and Statement by the Executive Director for Madagasear}

In the context of the fourth review under the Poverty Reduction and Growth Facility and requests for extension of the arrangement and additional interim assistance under the enhanced Initiative for Heavily Indebted Poor Countries, the following documents have been released and are included in this package:

- the staff report for the fourth review under the Poverty Reduction and Growth Facility and requests for extension of the arrangement and additional interim assistance under the enhanced Initiative for Heavily Indebted Poor Countries, prepared by a staff team of the IMF, following discussions that ended on November 14, 2003, with the officials of the Republic of Madagascar on economic developments and policies. Based on information available at the time of these discussions, the staff report was completed on March 2, 2004. The views expressed in the staff report are those of the staff team and do not necessarily reflect the views of the Executive Board of the IMF.

- a staff supplement of March 15, 2004 related to a request for increased access under the Poverty Reduction and Growth Facility.

- a staff statement of March 17, 2004 updating information on recent developments.

- a Press Release summarizing the views of the Executive Board as expressed during its March 17, 2004 discussion of the staff report that completed the review and requests.

- a statement by the Executive Director for the Republic of Madagascar.

The documents listed below have been or will be separately released.

Letters of Intent and Supplementary Letter of Intent sent to the IMF by the authorities of the Republic of Madagascar*

Memorandum of Economic and Financial Policies by the authorities of the Republic of Madagascar* Technical Memorandum of Understanding

* May also be included in Staff Report

The policy of publication of staff reports and other documents allows for the deletion of market-sensitive information.

To assist the IMF in eraluating the publication policy, reader comments are invited and may be sent by e-mail to publicationpolicy@imf.org.

Copies of this report are available to the public from International Monetary Fund - Publication Services $70019^{\text {th }}$ Street, N.W. - Washington, D.C. 20431

Telephone: (202) 623-7430 • Telefax: (202) 623-7201

E-mail: publications@imf.org Internet: http://www.imf.org

Price: $\$ 15.00$ a copy

International Monetary Fund

Washington, D.C. 
INTERNATIONAL MONETARY FUND

REPUBLIC OF MADAGASCAR

\title{
Fourth Review Under the Poverty Reduction and Growth Facility and Requests for Extension of the Arrangement and Additional Interim Assistance Under the Enhanced Initiative for Heavily Indebted Poor Countries
}

\author{
Prepared by the African Department \\ (In consultation with the Finance, Fiscal Affairs, Legal, Monetary and Financial Systems, \\ Policy Devclopment and Review, and Statistics Departments)
}

Approved by A. Basu and Martin J. Fetherston

March 2, 2004

- Social and political stability has been broadly restored, but limited political unrest in Toamasina, the largest port of the country, has been reported. Turnout at the local elections in November 2003 was about 50 percent, with the Prcsident's party receiving 56 percent of the votes.

- The third review under the Poverty Reduction and Growth Facility (PRGF) arrangement was concluded on June 30, 2003 (EBS/03/81, 6/12/03). Discussions on the fourth review under the PRGF arrangement were held during October 6-21, 2003 in Antananarivo and November 9-14 in Washington, D.C. The principal Malagasy representatives included Mr. Benjamin Radavidson, the Minister of Economy, Finance, and Budget, and Mr. Gaston Ravelojaona, Govemor of the Central Bank of Madagascar. The mission was received by the President, Mr. Mare Ravalomanana. The mission also met with representatives of the trade unions, and the business and donor communities.

- The staff team comprised Messrs. Vaez-Zadeh (head), Xie, Nassar (all AFR), Nascimento (PDR), and Jahjah (Resident Representative), and Ms. Jouanin (Assistant-AFR). Ms. Andriamihaja (Economist, Resident Representative Office) provided valuable assistance to the mission. Mr. Fayolle (AFR) joined the mission in the second week. The mission worked closely with the World Bank staff based in Madagascar.

- In the attached letter of intent and memorandum on economic and financial policies (MEFP) dated March 2, 2004 (Appendix 1L), the Minister of Economy, Finance, and Budget and the Governor of the Central Bank of Madagascar (BCM) review progress under the 2003 program and outline the government's economic program and the policies for 2004 . They request interim assistance under the enhanced Initiative for Heavily Indebted Poor Countries (HIPC Initiative), an extension of the PRGF arrangement until March 1,2005, and rephasing of the disbursements for July 2004 and February 2005.

- The schedule of future disbursements and program reviews is presented in Table 1. 


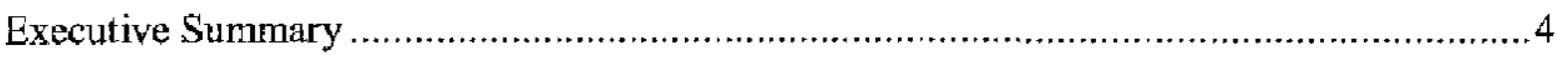

Economic Developments in 2003 and Performance Under the Program ................................. 5

A. Growth and Inflation ..................................................................................... 5

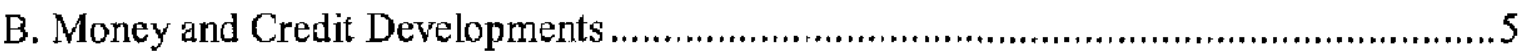

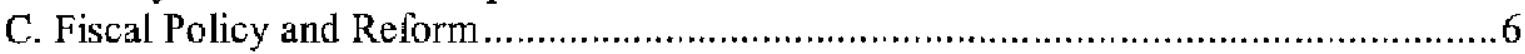

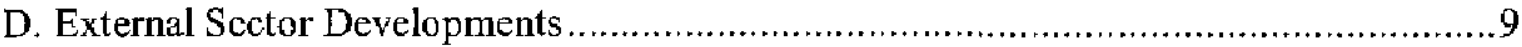

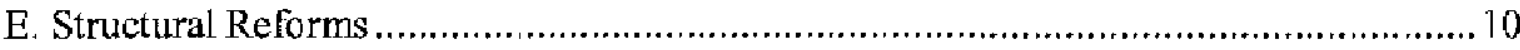

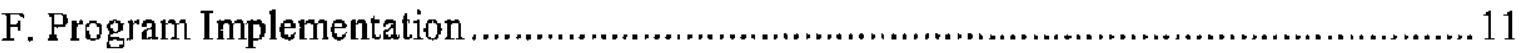

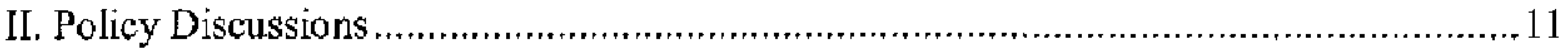

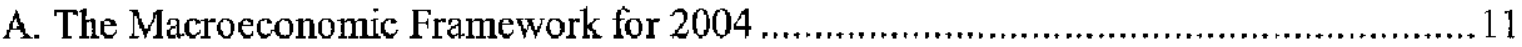

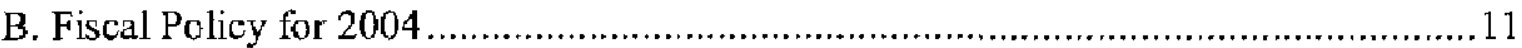

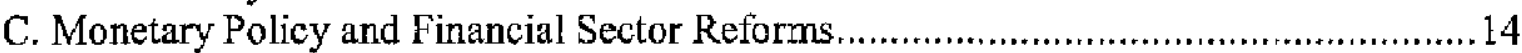

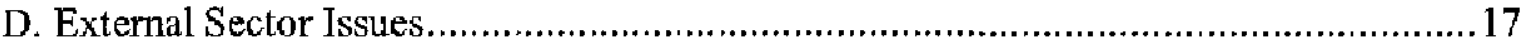

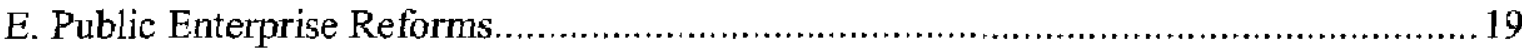

III. The PRSP and the Medium-Term Framework ,.......................................................19

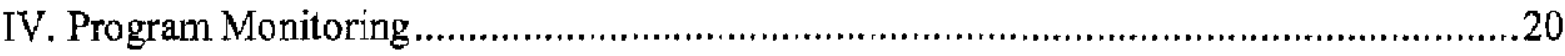

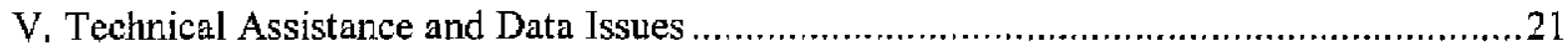

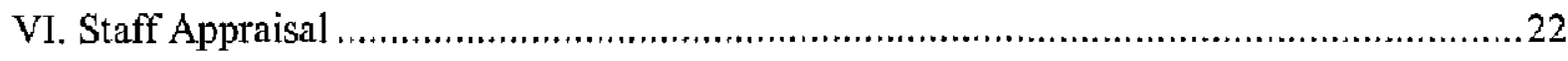

$\underline{B o x e s}$

1. Magnitude of the Exemptions (including VAT) Granted in August 2003 …..............7

2. Bank Soundness Indicators, December 1998 to Junc 2003 ................................. 16

3. Structural Conditionality .........................................................................21

$\underline{\text { Tables }}$

1. Tentative Work Program Under the Proposed Revised

PRGF Arrangement, 2003-05 .....................................................................26

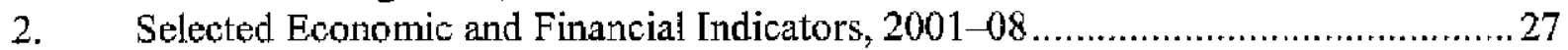

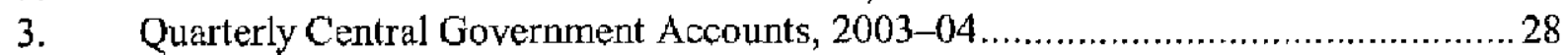

4. Central Government Accounts, 2002-08 (In billions of Malagasy francs) ..............29

5. Central Government Accounts, 2002-08 (In percent of GDP) ............................. 30

6. Monetary Survey, 2002-04,........................................................................... 31

7. Balance Sheet of the Central Bank and Consolidated Balance Sheet of

Commercial Banks, 2002-04 …................................................................. 32

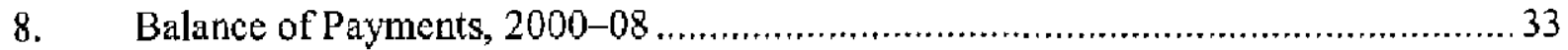

9. External Debt Sustainability Indicators, 2000-19............................................... 34 


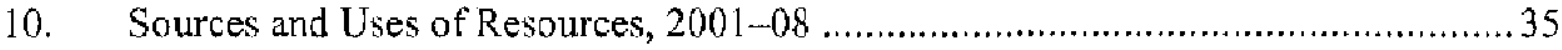

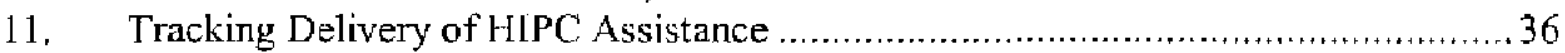

12. Regional Poverty by Area of Residence, 1993-2002 _........................................ 37

13. Basic Social and Demographic Indicators ...................................................... 38

14. Structural Performance Criteria, Structural Benchmarks, and Prior Actions for the

Fourth Review of the PRGF-Supported Program .................................................. 39

\section{Figures}

1. Consumer Price Index, February 2000 to October 2003 ...................................... 5

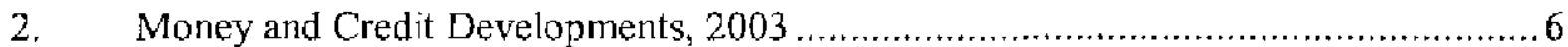

3. Weighted-Average Interest Rates, 1999:Q1-2003:Q3 ....................................6

4. Total Government Revenues, Cumulative Quarterly Data for 2003 ......................6

5. Government Current Expenditures, Cumulative Quarterly Data for 2003 ................

6. Government Capital Expenditures, Cumulative Quarterly Data for $2003 \ldots \ldots \ldots \ldots \ldots \ldots \ldots . .8$

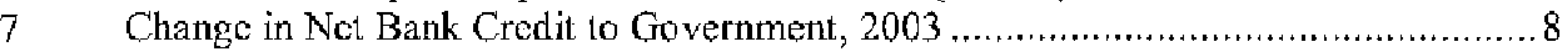

8. Total External Budgetary Support (Grants and Loans), 2003 _.............................. 8

9. Rcal and Nominal Exchange Rates, January 1996 - December 2003........................ 9

10. Change in Central Bank's Net Foreign Assets and Net Cumulative Purchase of

Foreign Currency, January - December 2003 ................................................2 25

\section{Appendixes}

I. Progress Toward HIPC Initiative Completion Point Triggers ...............................40

II. Status of Actions to Strengthen Tracking of Poverty-Reducing Public Spending ..... 43

III. Letter of transmittal .....................................................................................46

Attachment I: Memorandum on Economic and Financial Policies ......................47

Altachment II: Technical Memorandum on Monitoring the 2003-2004

Program Supported by the Arrangement under the PRGF .................................61

Attachment III: Notification of Currency Changeover.......................................6.6

IV. Relations with the Fund ........................................................................... 70

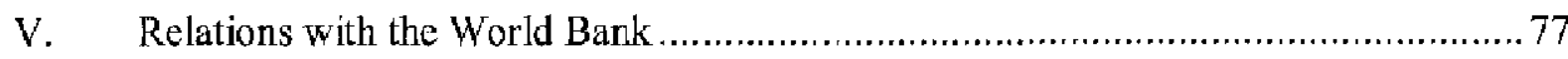

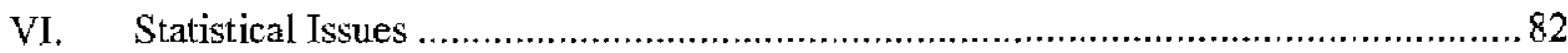




\section{EXECUTIVE SUMMARY}

- Madagascar's performance in 2003 under its Poverty Reduction and Growth Facilitysupported program was mixed. The inflation and growth outcomes for 2003 are likely to have been significantly better than expected. However, performance on the fiscal and external fronts is expected to have been weaker for the year as a whole, compared with the program. The overall fiscal deficit (on a commitment basis, excluding grants) is estimated to have reached 7.9 percent of GDP, compared with 7.6 percent under the program. The current account deficit (excluding official transfers) is estimated to have widened, owing mainly to a strong rebound in imports. The external reserves are estimated to have fallen to 3.1 months of imports, compared with 3.5 months of imports under the program.

- Fiscal performance deteriorated during the second half of 2003. Extrabudgetary expenditures amounting to 0.8 percent of GDP were incurred and a tax and tariff relief law enacted in August resulted in a shortfall in fiscal revenue relative to the program. Domestic arrears were accumulated, and there was substantial recourse to bank credit by the government during the second half of the year.

- On the structural front, privatization of public enterprises and some fiscal reforms are proceeding slowly, but progress has been made in improving efficiency and combating fraud and corruption in the tax and customs departments, although more effort is needed. The joint private/public sector committee overseeing these efforts has been instrumental in reducing cumbersome procedures. The tax department has also reinforced its tax collection units, especially as regards collections from large enterprises. Banking supervision is being strengthened, and banks remain well capitalized.

- The main objectives of the 2004 program are to achieve a real GDP growth rate of 6 percent, contain the annual inflation rate to about 5 percent, and increase international reserves to 3.8 months of imports. Tax reverue should reach 11.2 percent of GDP through simplifying tax and tariff structures, broadening the tax base, improving tax and customs administrations, and recovering tax arrears. The 2004 budget aims at raising the primary current surplus to 2.3 percent of GDP, and envisages a reduction in the government's net indebtedness to the banking system. An interbank foreign exchange market is scheduled to open by end-March 2004, and the privatization and rehabilitation of the communications, cotton, sugar, and utility companies will be completed by end2004.

- The full poverty reduction strategy paper (PRSP) was discussed at the Executive Boards of the IMF and the World Bank in November 2003, and the implementation of the policies contained therein has begun. 


\section{Economic Developments in 2003 and Performance Under the Program}

1. Overall economic performance in relation to the objectives and targets under the program was mixed during 2003. Real GDP growth is estimated to have exceeded the program target, and inflation was much lower than programmed. However, fiscal performance was weaker than programmed, as new exemptions reduced revenues, extrabudgetary expenditures were incurred, and domestic arrears accumulated. The balance of payments outcome is estimated to have been weaker than expected, with a larger current account deficit and substantial shortfalls in foreign financing. Structural reforms proceeded more slowly than planned, but progress was made in the anticorruption drive, reform of the tax and customs departments, and modernization of land ownership legislation. Apart from the nonobservance of the structural benchmark on the preparation of monthly treasury balance sheets up to end-2001, all other quantitative and structural performance criteria and structural benchmarks for end-June 2003 were met. The causes underlying the fiscal, balance of payments, and structural problems are explained in the rest of this section, and the views of the authorities and the staff on the policies to address them are presented in Section II.

\section{A. Growth and Innation}

2. There are indications that the economy is emerging from the 2002 economic stump, when real GDP declined by 12.7 percent following a period of serions political and social unrest (Table 2). In 2003, real GDP is estimated to have grown by 9.6 percent, compared with 6 percent under the program, reflecting recovery in the transport, construction, and tourism sectors as well as a rebound in agricultural production. At end-

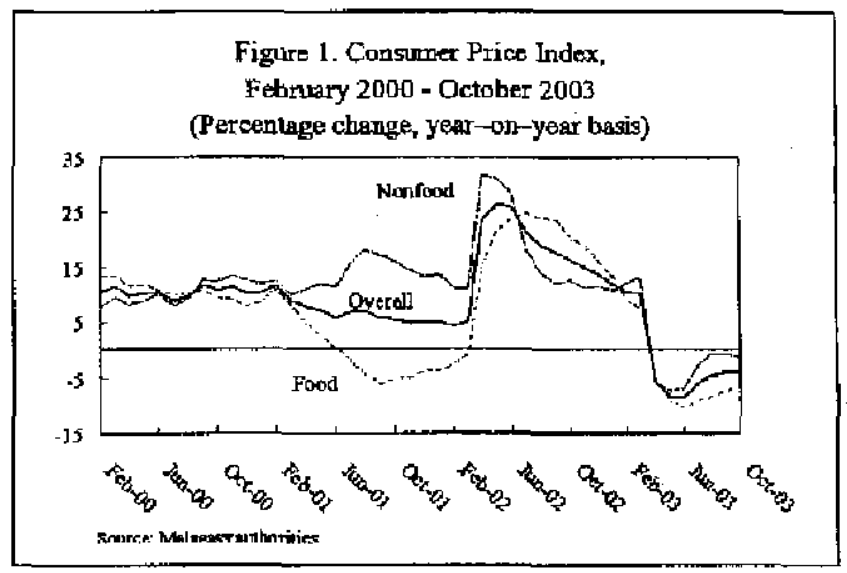
December 2003, the consumer price index (CPI) is estimated to have increased by 0.3 percent (on a year-on-year basis), against a program target of 7 percent. The main factors explaining the fall in the inflation rate included (a) better harvests that contributed to a decline in food prices by over 10 percent (at end-June 2003 , on a year-on-year basis, Figure 1); (b) tight monetary conditions in the first half of the year; and (c) stable domestic prices of petroleum products resulting from the appreciation of the Malagasy franc (FMG) against the U.S. dollar.

\section{B. Money and Credit Developments}

\section{The money supply (M3) contracted during the first half of 2003, but grew} sharply during the second half. It is estimated to have increased by 12 percent for the year as a whole, compared with 13 percent under the program (Figure 2). This trend reflected, 
primarily, the movements in credit aggregates. Credit to the private sector was flat in the first half of the year, and net credit to government declined as the government repaid its statutory advances from the central bank mainly through issuance of treasury bills to the putlic. Net domestic credit rose significantly in the second half of the year, as crop financing grew strongly and govermment borrowing rose with the increased pace of government spending. All interest rates remained relatively stable (Figure 3) and substantially above the inflation rate throughout the year.

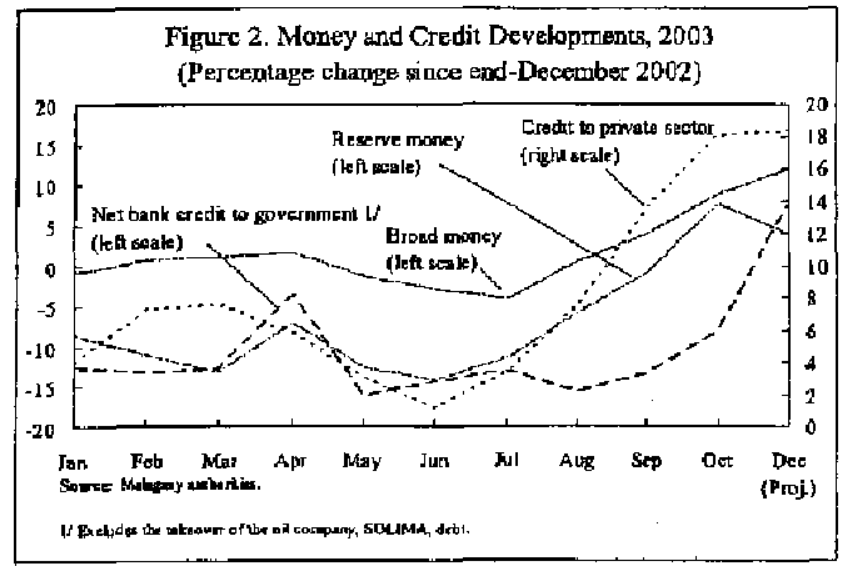

Figurt 3. Weighted Average Interest Rates, 1999:Q1-2003:Q3 (In percent)

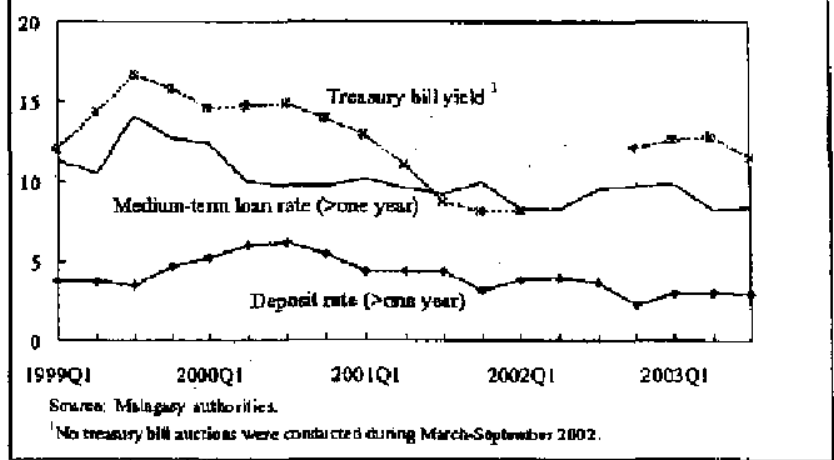

\section{Fiscal Policy and Reform}

\section{Fiscal performance was more favorable than expected under the program until} end-June 2003 , but fiscal slippages occurred during the rest of the year. Total government revenue, which was 4 percent higher than program projections at end-June 2003 , is est imated to have been 3 percent lower than the program by year's end (Figure 4), owing mainly to the impact of the tax and tariff exemptions announced by the President in July and enacted into law in August 2003.

The law exempted the imports of capital goods and selected other commodities from all tariffs as well as the value-zdded tax (VAT)

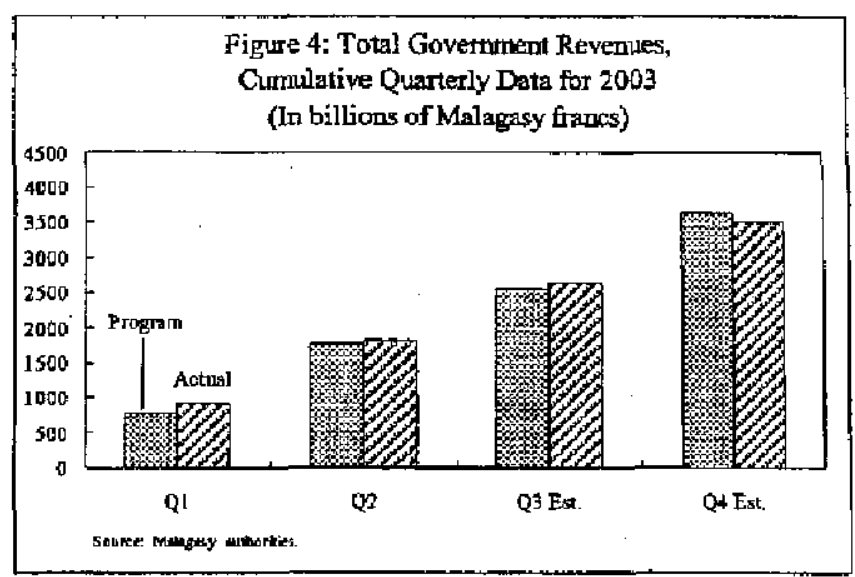
for a period of two years, effective September 1, 2003. The fiscal impact of these exemptions is estimated at 0.24 percent of GDP for the last four months of 2003 (Box 1). Besides the slippage in tax revenue, 
extrabudgetary expenditure amounting to 0.8 percent of GDP was incurred in the second half of the year, ${ }^{1}$ Reflecting these developments, current expenditure increased by 1.6 percent

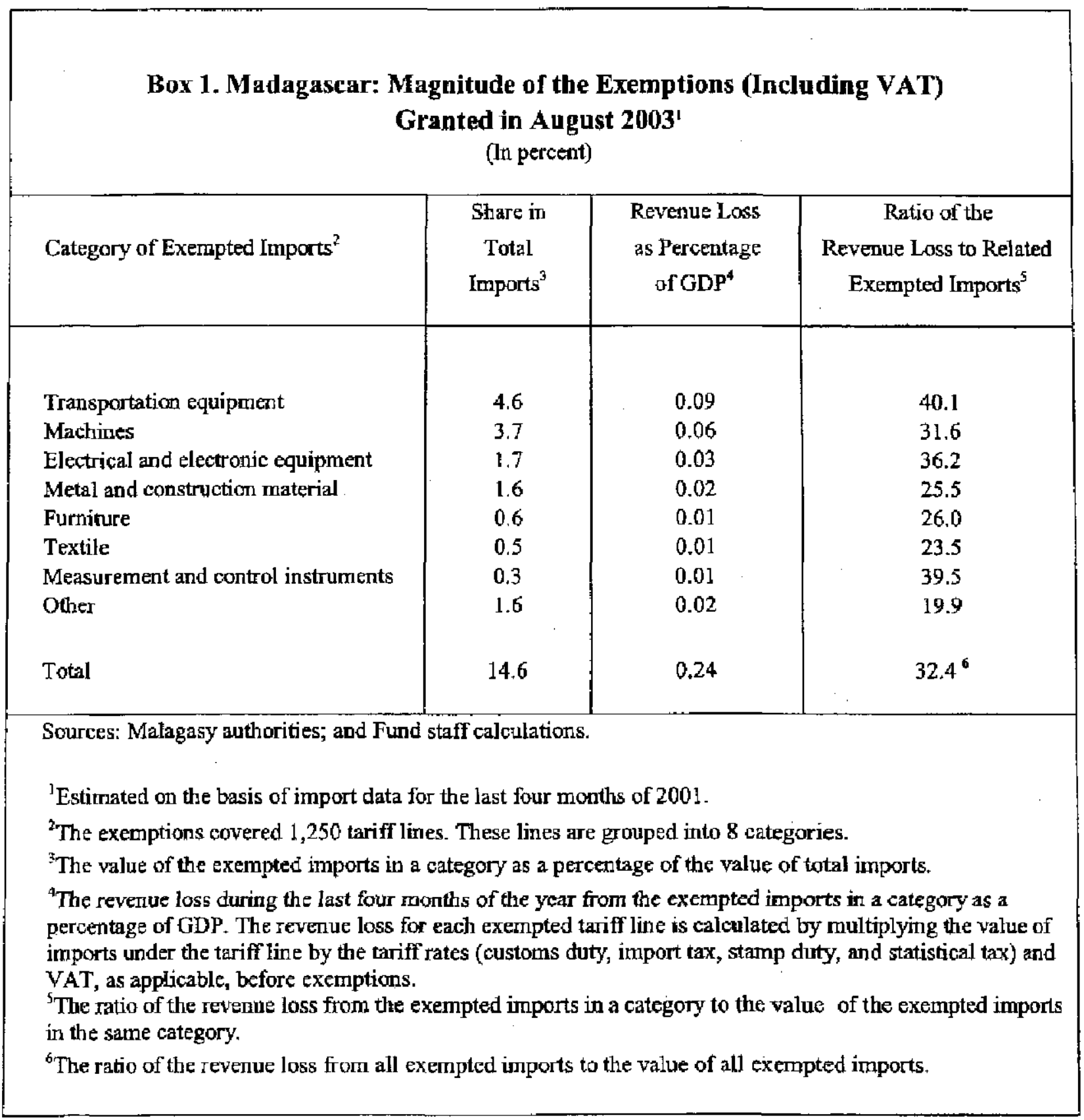

${ }^{1}$ The extrabudgetary expenditure included additional indemnities for the military, additional scholarships for university students and overtime pay for teachers, additional payments to Lufthansa Consulting for its management of Air Madagascar, and several other items. 
of GDP above the programmed amount (Figure 5) and additional domestic interest obligations (of about 0.7 percent of GDP) arose as the government issued treasury bills--some with longer maturities ( 52 weeks) carrying higher interest rates-to repay the central bank. ${ }^{2}$ Nevertheless, total government expenditure is estimated to have been slightly below the program, as limited implementation capacity led to lower investment outlays (Figure 6).
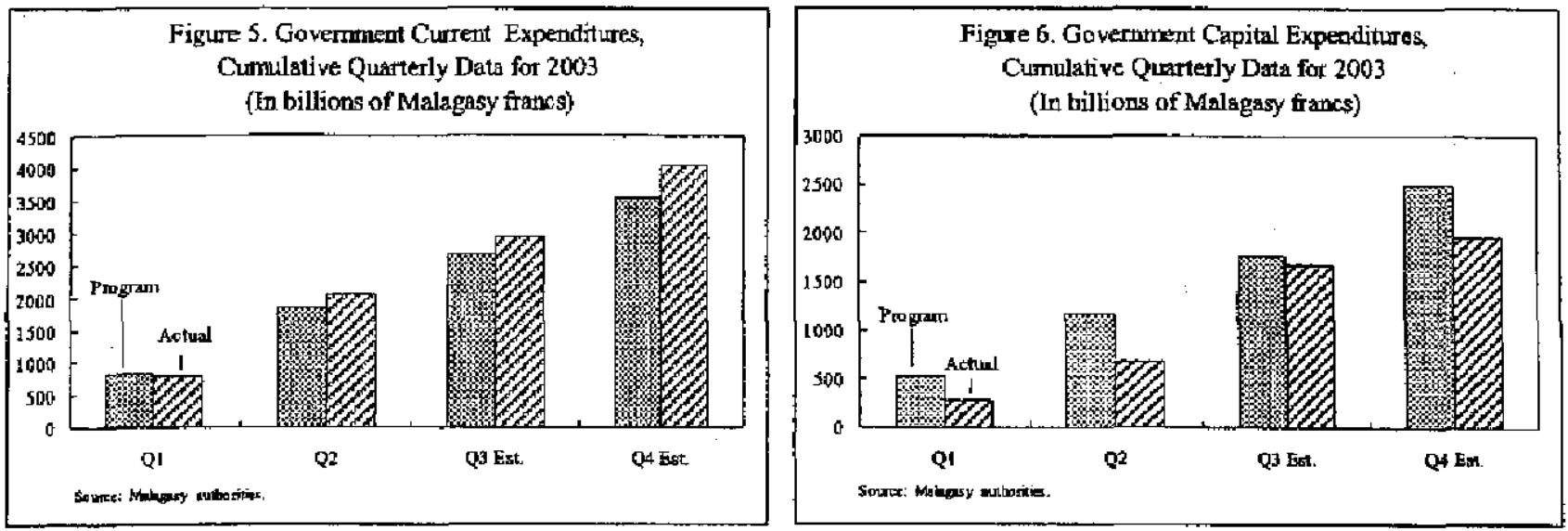

5. The overall fiscal balance in $\mathbf{2 0 0 3}$ is estimated to have deteriorated by the end of the year, requiring substantial recourse to bank credit (Figure 7). The deficit on a commitment basis, excluding grants, is estimated to have amounted to 7.9 percent of GDP, compared with 7.6 percent under the program. With external budgetary grants and loans
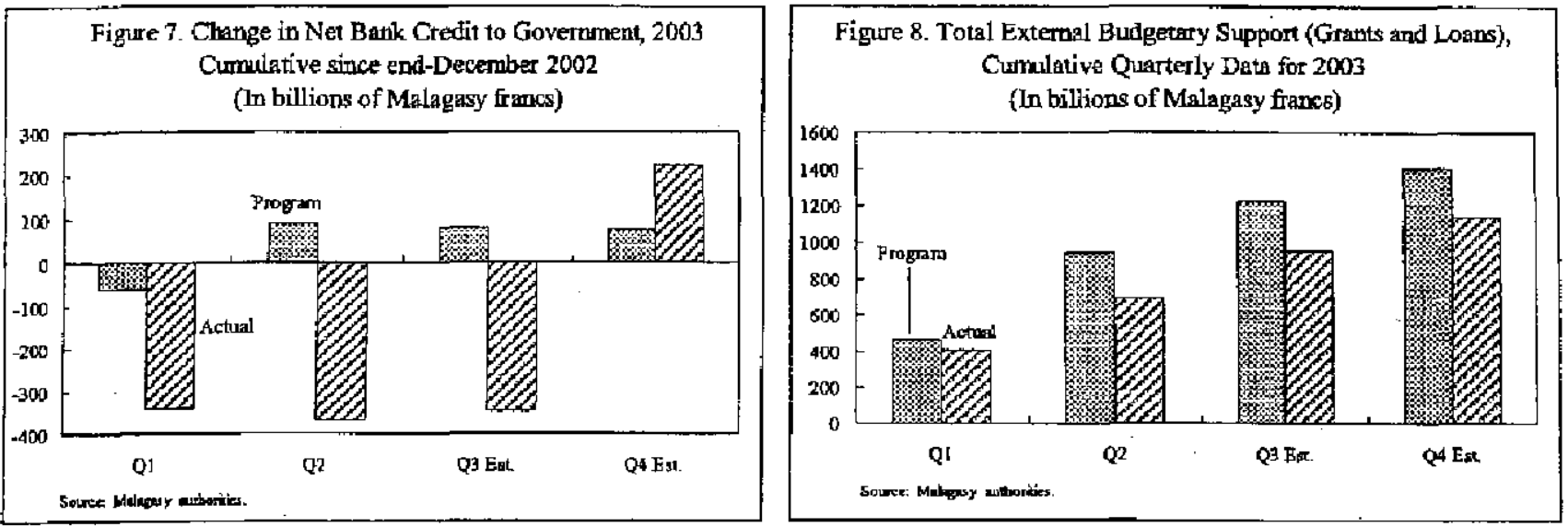

(Figure 8) falling short of the program, domestic financing is estimated to have amounted to FMG 710 billion (2 percent of GDP), almost five times the amount programmed (Table 4). The authorities indicated that, as a corrective measure, they had reinforced tax recovery efforts before the end of the year, but feared that additional revenue could be realized only in

${ }^{2}$ The average interest rate on these securities was about 4 percentage points higher than cnvisaged under the program. 
early 2004 . They pointed out that blocking nonpriority expenditure was not feasible, since all expenditure for 2003 had been already commitled by end-October. With few other options to reverse the deteriorating fiscal trends, the authorities noted that the end-December performance criteria on domestic financing of the budget and net domestic assets of the central bank were likely to have been missed. However, they resolved to restore fiscal discipline in 2004 through fiscal policy and budgetary control measures. They agreed with the staff's proposal that progress in the implementation of these policies and measures be monitored closely through the establishment of quantitative performance criteria for endMarch 2004.

\section{External Sector Developments}

\section{The balance of payments outcome for 2003 is estimated to have been less} favorable than expected under the program. The external current account deficit is estimated to have widened to SDR 193 million (4.9 percent of GDP), compared with SDR 169 million under the program, despite a sizable improvement in the terms of trade. The balance of payments outcome reflects (a) weaker than expected recovery of EPZ and cotton exports; (b) a strong rebound in imports, and (c) lowerthan-expected disbursement of budgetary aid. International reserves at year's end are

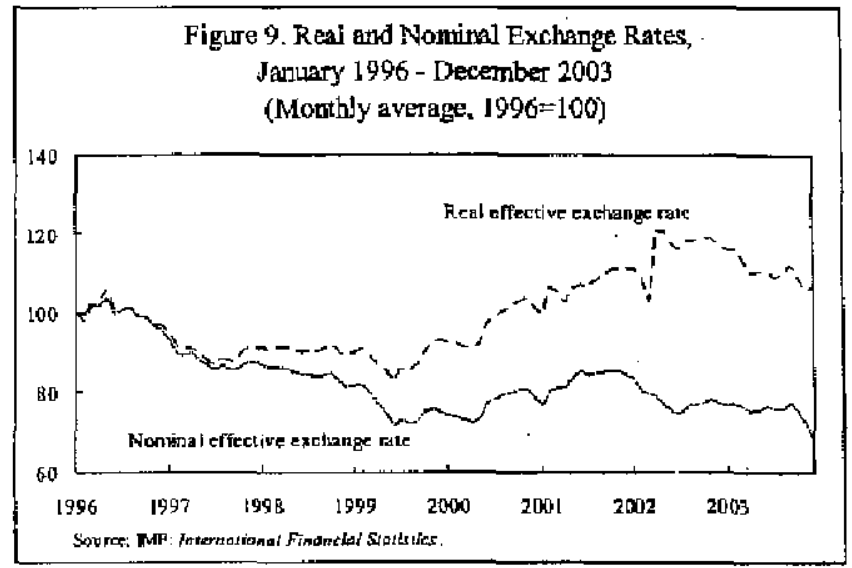
estimated to have fallen to about 3.1 months of imports, compared with 3.5 months envisaged under the program. The real effective exchange rate, which had appreciated significantly during 2000-02, ${ }^{3}$ depreciated by about 11 percent in 2003 (Figure 9), mainly reflecting nominal exchange rate movements (in particular against the strengthening euro). This real depreciation partially reversed the adverse impact of the earlier appreciation on export competitiveness. ${ }^{4}$

\footnotetext{
The real effective exchange rate appreciated cumulatively by 34 percent from January 2000 to December 2002, reflecting the higher inflation rate in Madagascar, compared with its trading partners. The real appreciation during 2000-01 (but not in 2002) also reflected the appreciation of the nominal effective exchange rate caused by increasing foreign direct investment inflows associated with the export processing zone.

${ }^{4}$ Export volume growth had decelerated from 26.3 percent in 2000 to 9.5 percent in 2001 . During the 2002 political crisis, export volume declined by 52 percent.
} 


\section{E. Structural Reforms}

7. Progress on privatization of public enterprises was slow. The divestiture contract for the telecommunications company, TELMA, was signed in August 2003 between the government and DISTACOM, but the parties are awaiting completion of due diligence, and discussions are going on with the minority shareholder to sell its shares. In the case of the utility company, JRAMA, the report of the World Bank-financed study on the company's financial situation was delayed. As for the sale of the cotton company, HASYMA, the bidding contract had to be revised to accommodate the current minority shareholder's preference to sell its shares if it does not win the bid. The tender of a management contract for the sugar company, SIRAMA, was nullified in June 2003 due to bidding irregularities.

\section{A number of measures were introduced in 2003 to enhance private sector} confidence. First, an anticorruption commission was established in May, and its first report was issued in November. The report, which was presented to the government, private sector, media, and donors, provides a detailed account of comuption in the judiciary, the police department, customs, and government contracting, and proposes specific recommendations to rectify the situation. S Second, progress has been made in improving efficiency and combating fraud and corruption in the tax and customs departments. The joint private/public sector committee overseeing these efforts has reduced cumbersome procedures at the customs, and a one-stop counter has been created to handle all clearance-related operations. The tax department has also reinforced its tax collection units, especially as regards collections from large enterprises and the recovery of tax arrears. Third, the Property Act was amended in August 2003 to allow foreign investors to own land. The law is expected to facilitate the use of land as collateral by foreign banks.

9. The authorities are introducing a new currency (the ariary) and have established a three-stage conversion procedure in accordance with the recommendations of the Monetary and Financial Systems Department (MFD) (MEFP, para. 12). ${ }^{6}$ In the first stage, which began on July 31, 2003 and is proceeding smoothly, new ariary notes are gradually being put into circulation as and when currency withdrawals are requested by banks and the trcasury. During this stage, the two currencies, the Malagasy franc and ariary, will circulate together, but Malagasy franc notes will not be exchanged for the ariary. These exchanges will be permitted during phase two, which begins in November 2004 and extends to end-2009. While no exchanges will be possible after end-2009, Malagasy franc notes will continue to be legal tender indefinitely. All accounts will be held in Malagasy franes until end-2004 and

\footnotetext{
${ }^{5}$ The report was prepared by foreign consultants and supported by the European Union (EU) and Transparency International.

${ }^{6}$ The authorities explained that the currency changeover was motivated by the desire to revert to the old name of the monetary unit, the ariary. The changeover was also seen as an opportunity to put into circulation banknotes containing more security features.
} 
converted into ariary on January 1, 2005 (phase three). The authorities have sent a formal notification of the currency change to the Fund (Appendix III, Attachment III).

\section{F. Program Implementation}

10. All quantitative and structural performance criteria and benchmarks for endJune 2003 were met (Table 14 and Appendix II, Attachment I, Table 1), with the exception of the structural benchmark on the preparation of monthly treasury balance sheets up to end-2001. However, annual balance sheets were prepared up to end 2001 . The treasury will prepare quarterly balance sheets beginning in 2004, to be followed by monthly balance sheets at a later stage. Preliminary estimates indicate that the indicative targets for end-September 2003 were also met, except that on the domestic financing of the budget.

\section{Policy Discussions}

11. The discussions focused on measures aimed at restoring fiscal discipline, ensuring growth and stability, and increasing poverty-related expenditures. The authorities intend to enhance transparency and efficiency in public resource management to improve governance, reorient their investment effort toward the health, education, transport, and rural development sector to strengthen the impact on poverty reduction, and erihance the role of the private sectors in the economy. They also plan to accelerate the pace of structural reforms, especially in the areas of fiscal management, public enterprise reform, credit and money market development, and the legal and regulatory framework affecting the business environment. The program for 2004 sets out the main policies in these areas.

\section{A. The Macroeconomic Framework for 2004}

12. The macroeconomic framework for 2004 seeks to achieve real GDP growth of 6 percent, contain the annual consumer price inflation rate at 5 percent (end-of-year basis), and increase international reserves to 3.8 months of imports. As explained in the staff report for the third review (IMF Country Report No. 03/203, 7/3/03), the growth target falis between the high and low projections in the poverty reduction strategy paper (PRSP). It is based on an investment implementation ratio that is somewhat higher than that of 2003, as the authorities are introducing measures to enhance the country's absorptive capacity (see para. 17).

\section{B. Fiscal Policy for 2004}

13. The $\mathbf{2 0 0 4}$ budget aims at raising the primary current surplus from 0.7 percent of GDP in 2003 to 2.3 percent of GDP in 2004. To achieve this objective, the authorities intend to raise the tax revenue-to-GDP ratio from 10 percent in 2003 to 11.2 percent in 2004 and reduce noninterest current expenditure (Table 5). However, on a commitment basis and excluding grants, the overall buctget deficit will improve only slightly, to 7.8 percent of GDP, largely due to a 56 pereent increase in (mainly foreign-financed) investment expenditure. 
With an expected increase in total grants to 5 percent of GDP (from 4 percent of GDP in 2003 ), ${ }^{7}$ the overall budget deficit (on a cash basis and including grants) will be reduced further to 3.7 percent of GDP, compared with 4.6 percent of GDP in 2003. Given the expected external financing, the government will reduce its net indebtedness to the banking system.

14. Achieving the tax revenue target for $\mathbf{2 0 0 4}$ is likely to present a challenge, especially as the August tax and tariff relief law is expected to reduce 2004 revenues by about 1 percent of GDP (about 9 percent of total tax revenue). However, although ambitious, the target for the tax revenue-to-GDP ratio remains below the level attained in 2000. Moreover, the measures included in the 2004 budget law are expected to yield 0.7 percent of GDP in additional revenue. These measures are aimed at lowering tariff barriers to enhance the openness of the economy, broadening the revenue base, simplifying the tax system, and maintaining the integrity of the VAT (MEFP, Table 3). ${ }^{8}$ As part of these measures, effective January 1, 2004, the four trade taxes (customs duty, import tax, statistical tax, and stamp duty) were collapsed into two (customs duty and import tax), ${ }^{9}$ and the number of tariff rates was reduced from seven $(3,8,13,18,23,28$, and 33 percent) to four $\left(5,10,20\right.$, and 25 percent) ${ }^{10}$ The 2004 budget also extended the VAT to land passenger transport and all nonprocessed agricultural products (except rice), raised the excise tax on tobacco and cigarettes from 70 percent to 80 pencent, and eliminated the costly tax reduction for investment. In addition to these revenue measures, the authorities are strengthening the efforts to collect taxes due and recover tax arrears. They are also intensifying the administrative reform of the tax and customs departments to reduce fraud and corruption (MEFP, para. 39).

15. The staff supported the authorities' emphasis on trade liberalization, but cautioned that the tax exemptions could pose several problems. First, the tax exemptions make the tax system more complex to administer and reduce the efficiency of the VAT. Second, the discretionary nature of ad hoc exemptions could create policy uncertainty and

${ }^{7}$ The World Bank is expected to provide budgetary grants for the first time, amounting to US\$50 million in 2004.

${ }^{8}$ These measures are largely based on the recommendations of the July 2003 tax policy mission of the Fiscal Affairs Department (FAD). However, the August tax and tariff exemptions were not recommended by FAD.

${ }^{9}$ These two taxes are expected to be merged into a single tax in 2005 .

${ }^{10}$ Following the tariff reforms, imports have been classified into four categories. Each category is now subject to two taxes, namely, customs duty and import tax, the sum of which amounts to $5,10,20$, and 25 percent, depending on the category of imports. As a result, the weighted-average tariff rate, which was estimated to be about 18 percent before the tariff reforms, is projected to drop to around 14 percent (based on 2001 import data). 
opportunities for rent seeking. Third, pressures for more exemptions may continue, especially because some consumer durables also benefited from the exemptions. ${ }^{11}$ Fourth, in anticipation of further exemptions, some final goods producers may decide to delay imports and production. Fifth, the temporary exemptions may prove politically difficult to remove as planned, thereby reducing policy credibility. The staff suggested that a comprehensive, stable, and transparent system of low tariffs for capital goods imports would be more credible and provide better incentives for investment. The authorities responded that the exemptions are intended to provide a temporary incentive for investment and induce high growth. They emphasized that no new exemptions would be granted beyond those included in the August 2003 tax and tariff relief law and the 2004 budget law (a structural performance criterion).

16. The 2004 budget limits the growth of current expenditure to 6.6 percent. This increase reflects (a) an increase in domestic interest obligations as government debt to the central bank is converted into marketable securities; ${ }^{12}$ (b) the inclusion in the budget of realistic estimates of the fees to be paid for preshipment inspection of imports; ${ }^{13}$ (c) larger allocations for road maintenance; and (d) an increase in the wage bill of about 2.3 percent. ${ }^{14}$ The authorities explained that the small growth in the wage bill, which follows a sharp increase in 2003, is intended to allow the expansion of other nonwage priority (including investment) expenditures. The increase in the wage bill reflects the recruitment of teachers, doctors, security personnel, and judges needed for the implementation of the priority PRSP projects in the corresponding sectors. The increase also accommodates incentives for voluntary early retirement, which is a key initiative to strengthen control over personnel expenditure in the medium term. The limited increase in the wage bill leaves no room for a general salary increase, but bonuses will be granted based on merit.

17. The rate of implementation of investment projects is expected to increase above the level in 2003 ( 75 percent), allowing capital spending to rise from 5.8 percent of GDP in 2003 to 8.2 percent of GDP in 2004. Much of this increase will cover the priority sectors of health, education, transport, and rural development. In order to achieve the higher

${ }^{11}$ Additional exemptions are included in the budget law of 2004 for medical products, mosquito nets, and tractors. The fiscal impact is estimated at below FMG 10 billion.

${ }^{12}$ The current interest rate on the FMG 1,620 billion of government debt to the $\mathrm{BCM}$, being converted into marketable securities, is 1 percent per year, substantially below the prevailing market rate. However, the net impact of the conversion on the budget deficit will be minimal as the $\mathrm{BCM}$ has agreed to transfer its net income arising from the conversion to the treasury on a regular and frequent basis.

${ }^{13}$ These fees were underbudgeted in the past, resulting in payment arrears.

${ }^{14}$ The wage bill increased by 37 percent in real terms in 2003 . The ratio of the wage bill to GDP rose from 4.6 percent in 2002 to 5.6 percent in 2003 . It declines to 5.1 percent in 2004 . 
implementation ratio, the procurement process for public projects has been modified to reduce delays, and efforts are under way to improve coordination and communication among the Directorate of Public Investment, the General Directorate of Public Expenditures, and the donors.

18. The government plans to clear all payment obligations outstanding at the end of 2003, before end-March 2004, ${ }^{15}$ and pay the arrears of SIRAMA and TELMA (totaling 0.4 percent of GDP) to sugar planters and creditors, respectively (MEFP, para. 23). To prevent the occurrence of new arrears, administrative sanctions, including financial penalties, for infraction of government regulations that have not been properly enforced in the past, will be strictly applied against ministries and public agencies that exceed committed expenditures. Moreover, the planned establishment of an integrated public finance management system linking the treasury and expenditure control department will facilitate the monitoring of arrears. It is proposed that nonaccumulation of arrears be monitored by a performance criterion.

19. Efforts to improve treasury accounting, simplify the budget execution system, and strengthen the monitoring of budget implementation (Appendix II) will be intensified. For technical reasons, the extension of the integrated public finance management system of 'Toamasina to Antananarivo, and the planned linking of the two systems, did not take place before end-2003 as scheduled. However, it is expected that the system will be operational in Antananarivo soon, and extended to other provinces by end-2004. The delay in the preparation of the treasury monthly accounts will be reduced to 15 days in areas where the integrated system has been established. The quarterly "flash" reports on budget execution, which now cover six ministries, will be extended to cover all ministries in 2005. In order to better control spending toward the end of the year, the government has moved the deadline for expenditure commitments by one month from November 30 to October 30 , and all payments will be made before end-December. Moreover, government expenditure will be cut and administrative measures taken, should revenue projections prove optimistic. The authorities also affirmed their intention not to incur extrabudgetary expenditures.

\section{Monetary Policy and Financial Sector Reforms}

20. Consistent with the growth and inflation objectives for 2004, broad money (M3) is programmed to rise by about 12 percent during the year. This objective will provide ample room for the extension of credit to the private sector, as there will be no recourse to net bank credit by the government in 2004. Demand for credit by the private sector is expected to rise with the improved investment climate and the resumption of economic activity.

${ }^{15}$ These payment obligations include float items. They are considered arrears if not cleared within the regulatory period of three montbs and will be monitored by the proposed performance criterion on nonaccumulation of arrears. 
21. To improve the effectiveness of monetary policy in maintaining low inflation, the BCM will further strengthen liquidity forecasting (MEFP, para. 34) and facilitate the development of the money market. The monthly liquidity forecasts will be refined and weekly forecasts (in line with the weekly primary auction of treasury bills) developed to provide a basis for the BCM's interventions in the money market to regulate banks' reserves. The planned conversion of long-outstanding government debt to the central bank into marketable securities will eventually allow the BCM to conduct open market operations. The BCM intends to introduce such operations gradually, laking into account the impact of the sale of government securities it holds on budgetary costs. Efforts are also needed to deepen the money market further by improving the maturity range of the instruments available. To this end, two issues of two- and three-year government bonds were sold on tap in August and September 2003. However, the bonds did not generate interest because they carried relatively low interest rates of 5 percent and 6 percent, respectively. No new issues of these bonds are planned for 2004 .

22. The authorities intend to take further steps to improve the availability of credit, especially to the small- and medium-sized enterprises. The commercial banks have maintained substantial excess reserves and remained cautious in lending to the private sector, owing to, among other things, exposure limits imposed by head offices, ${ }^{16}$ problems with the availability and seizure of collateral, and growing nonperforming loans. The amendment of the Property Act should alleviate the collateral problem, but its full impact will only be realized once an appropriate land registry system is in place. This reform is expected to be completed in 2004. The authorities also intend to streamline the procedures for the use of the Loan Guarantee Fund, to allow coverage of more loans. ${ }^{17}$ To help the expansion of microfinance operations, plans are being finalized for their registration and regulation, and advisory services (on credit evaluation, accounting, loan recovery, organization, and management) will be offered to the registered microfinance institutions through a World Bank-financed project.

23. Banks are well capitalized and their profits rose in 2003, but the share of nonperforming loans in their portfolios has continued to grow (Box 2). The authorities believe that nonperforming loans will decline as medium-sized and large enterprises recover from the 2002 economic downturn. Nevertheless, they are also strengthening

${ }^{16}$ Six of the seven commercial banks are foreign owned.

17 The fund was set up with donors' assistance in March 2003 to reduce banks* risks in lending to small- and medium-sized enterprises. By the end of September 2003, 20 loans had received guarantees, amounting to a total of FMG 13.9 billion, or about 55 percent of total loan principal. 


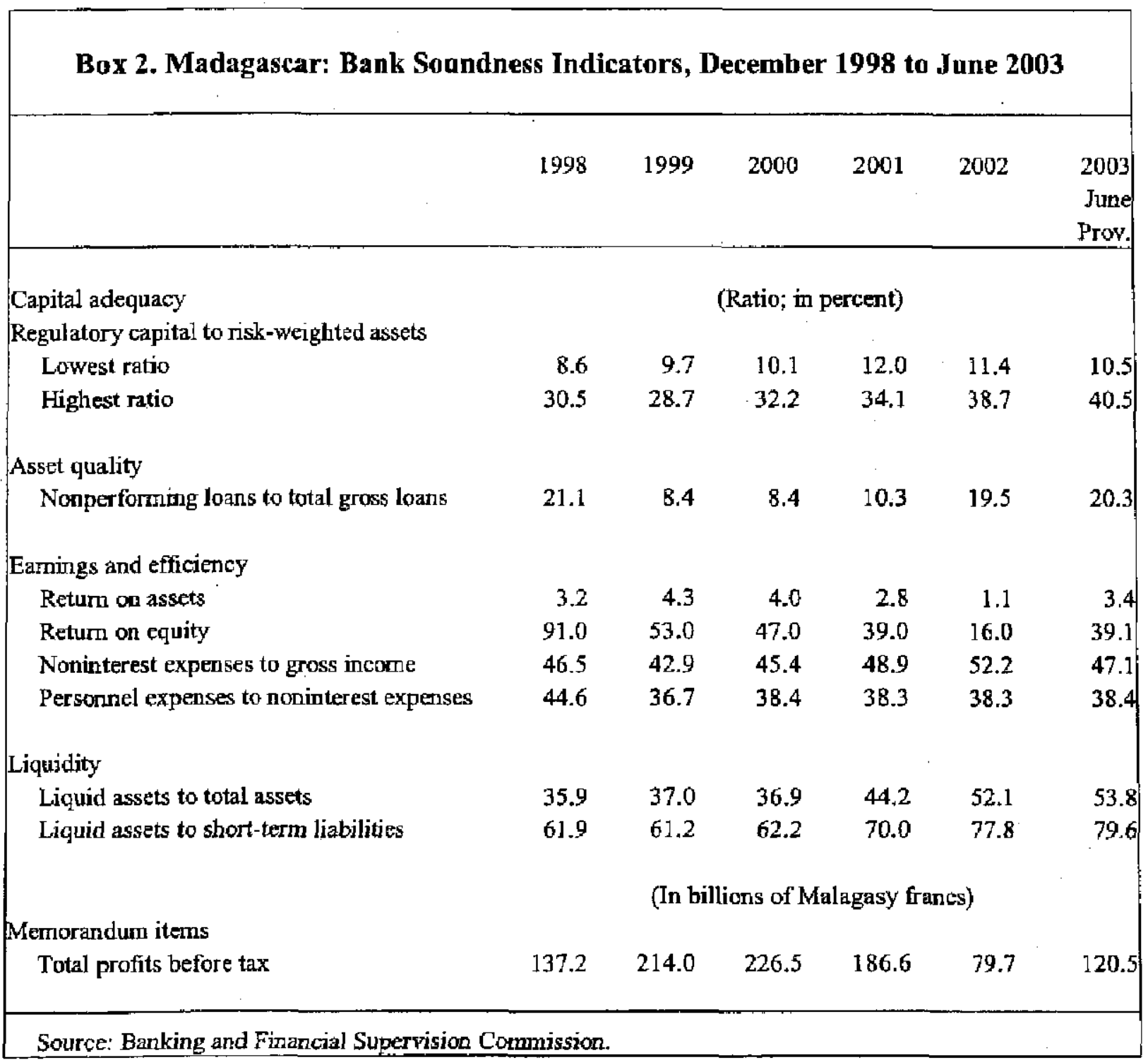

banking supervision, developing an early warning system for bank fragility, with the assistance of MFD, and establishing closer cooperation with the supervisory agencies in commercial banks' home countries. The mission stressed that the reform of the judicial system and the regulatory framework for collateral seizure and bankruptcy should be pursued as well.

24. Progress has been made in implementing the main recommendations of the safeguards assessment report (MEFP, para. 37). The 2004 budget law mandates the conversion of the government's long-standing debt to the BCM (for which no repayment schedule exists) into marketable securities at the beginning of 2004 (MEFP, para. 36). The audif of reserve management and foreign exchange activities, a structural benchmark, has begun with the assistance of MFD. In addition, anti-money-laundering legislation has been enacted. The authorities are preparing an action plan for the implementation of the remaining safeguards requirements, especially the modernization of the central bank's accounting framework. 


\section{External Sector Issues}

25. The external current account deficit (excluding official transfers) is projected to widen slightly to 7.6 percent of GDP in 2004. Imports are projected to grow by 16 percent, owing to the authorities' ambitious public investment program, the effects of the August tax and tariff relief law, and the impact of the trade liberalization measures included in the 2004 budget. ${ }^{18}$ Exports are expected to grow by no more than 8 percent in 2004 , held back by a drop in vanilla exports, ${ }^{19}$ while net services and factor income flows strengthened somewhat. Reflecting increased external assistance, the gross international reserves of the central bank are projected to reach 3.8 months of imports (compared with an estimated 3.1 months of imports in 2003).

\section{The authorities emphasized their intention to allow the exchange rate to be} market determined. The flexible exchange rate poiicy will be accompanied by prudent macroeconomic policies aimed at keeping inflation low and structural reforms to improve the efficiency of the export sector, thereby helping to maintain Madagascar's external competitiveness. The central bank will only intervene in the foreign exchange market to dampen sharp swings in the exchange rate, meet the floor on net foreign assets of the central bank under the PRGF-supported program, and facilitate official transactions (e.g., acquisition of foreign exchange for debt service payments). ${ }^{20}$ Preparations for the introduction of an interbank foreign exchange market are under way. The opening of the market, originally planned for end-December 2003, is now scheduled for end-March 2004 (a structural benchmark under the program) to allow for the computer network linking all banks and the BCM to be established and training of bank and BCM staff by professional foreign exchange dealers to be completed. To facilitate the operation of the market, a new foreign exchange control law is being drafted (MEFP, para. 31). Under the new law, the BCM will assume the responsibility for exchange rate policy and exchange control regulations.

27. The government has taken steps to liberalize international trade. Effective January 1, 2004, the tariff structure was simplified, and the weighted average tariff rate was lowered, accompanied by a reduction in the top tariff rate from 33 percent to 25 percent (see para. 14 above). Also, capital goods imports have been exempted from all taxes and tariffs, and the reform of customs operations and port procedures is under way. Procedures for approval of investment projects have been streamlined through the establishment of a one-

${ }^{18}$ Capital goods imports are projected to rise by 30 percent in 2004. About one third of this increase is attributable to the impact of the August tax and tariff relief law. Imports grew by 51 percent in 2003 after declining by 38 percent in 2002 in the aftermath of the crisis.

${ }^{19}$ Widespread concerns about security and theft led to premature harvesting of vanilla crops by farmers in 2003 , reducing the quantity of vanilla to be exported in 2004 .

${ }^{20}$ Figure 10 presents monthly purchases of foreign currency by the BCM. 
stop counter, where all formalities related to investment are taken care of. The cumbersome regulations on subcontracting of EPZ operations to small local firms have also been simplified to reduce the administraive burden on EPZ firms, while encouraging the growth of small local firms.

28. The promotion of EPZ exports, a priority for the government, will face challenges in the coming years. First, with the expiry of the World Trade Organization's (WTO's) Agreement on Textiles and Clothing by end-2004, large importers in the EU and the United States may reduce the number of source countries, and the incentives for "quota hopping" by investing in Madagascar will become less important. Second, the tariff preferences under the African Growth and Opportunities Act (AGOA) and the Everything But Arms (EBA) agreement will likely erode if multilateral tariffs decline in the context of the Doha round. These factors could lower Madagascar's textile exports. The authorities intend to respond to these challenges by implementing structural reforms, including infrastructural and transportation improvements and simplifications of customs procedures, aimed at reducing transaction costs of foreign trade and raising the efficiency of the export sector.

29. The regional integration agenda is being pursued. As a prelude to the launch of the customs union of the Common Market for Eastern and Southern Africa (COMESA) in December 2004, the government has taken several preparatory measures (MEFP, para. 27) and has approved membership in COMESA's Development Fund in 2004. It will also conduct a study on the impact of the adoption of COMESA's common external tariff on the competitiveness of Madagasear's main economic sectors, and will move toward the harmonization of the country's customs rules and procedures with those of COMESA. The regional integration agenda will take into account the framework for negotiations of the Economic Partnership Agreement with the EU scheduled to go into effect in 2008.

\section{Madagascar has finalized, or is about to finalize, bilateral rescheduling} agreements with most Paris Club and several non-Paris club creditors (Table 11). In particular, agreements with China and Kuwait have been finalized, and Madagascar has requested debt relief on terms comparable to Cologne terms from other creditors. Contacts have also been made with all official creditors for the reconciliation of outstanding debt in preparation for reaching the completion point under the enhanced HIPC Initiative,

\section{Based on a preliwinary analysis, the profile of external borrowing for 2005-08} remains broadly similar to that projected when Madagascar reached the decision point. For 2003-04, however, external debt indicators are projected to be weaker than decision point estimates, reflecting, inter alia; the adverse impact of the 2002 political crisis and lower vanilla exports. With the resumption of growth in 2003 and the expected rebound in exports 
in 2005, external debt indicators all tend to converge toward the decision point estimates over the medium term (Table 9). ${ }^{21}$

\section{E. Public Enterprise Reforms}

32. To speed up the privatization and rehabilitation of public enterprises, which have been proceeding at a slower pace than expected, the authorities are taking the following steps: (a) due diligence on TELMA will be completed in 2004; (b) the launching of the tender for the placement of JRAMA under a management contract was completed by end-Jamuary 2004 (a prior action under the program). The company will be placed under new management by end-June 2004 ; (c) the bidding process for the sale of HASYMA will be completed by end-March; and (d) the bidding process for placing SIRAMA under a management contract will be completed by end-June 2004. Given the urgency of public enterprise reforms to enhance productivity and reduce the quasi-fiscal costs (estimated to have amounted to 0.4 percent of GDP in 2003), and in view of the importance of the cotton and sugar sectors for poverty reduction, it is proposed that progress of the reforms concerning HASYMA and SIRAMA be monitored by structural performance criteria (MEFP, Table 2).

33. The authorities intend to allow the prices of petroleum products to reflect market conditions. Since the privatization of the state-owned oil company, SOLIMA, in 2001 , the government has set the price of petroleum products at the pump according to a predetermined schedule, agreed with the private oil companies that replaced SOLIMA. The schedule, which is updated on a monthly basis, takes into consideration petroleum import prices, taxes, and profit margins, among other factors. This price-setting mechanism, which is aimed at reducing fluctuations in the prices at the pump and was intended to be temporary, will be terminated by end-2004, allowing prices to be determined by market forces.

\section{IH. THE PRSP AND THE MEDIUM-TERM FRAMEWORK}

34. The sources of growth and policy priorities in the medium term are similar under the PRSP and the PRGF-supported program. ${ }^{22}$ Growth during 2005-08 will average 6 percent per year, spurred by robust expansion in the agriculture, construction, manufacturing, mining, and tourism sectors, where substantial potential for invesiment exists.

${ }^{21}$ An updated debt sustainability analysis (DSA) will be conducted in the coming months, in collaboration with the World Bank, in connection with the preparation of the HIPC Initiative completion point document.

${ }^{22}$ A full PRSP was submitted to the Fund and World Bank on July 30,2003 and discussed together with the Joint Staff Assessment (EBD/03/101) at the IMF Executive Board on November 21, 2003. Executive Directors concurred that the PRSP provided a comprehensive and coherent framework for guiding the implementation of the authorities' poverty reduction strategy. 
Boosted by trade liberalization and investment stimuli, both private and public investment are expected to increase relative to GDP (Table 10), assuming foreign direct investment and donor assistance materialize as expected and the pace of public sector project implementation accelerates.

35. The budgetary framework for the medium term aims at reducing the overall deficit (on a commitment basis and excluding grants) to about 7 percent of GDP in 2008 from 7.9 percent of GDP in 2003. This reduction will be consistent with growth, inflation, and balance of payments objectives over the medium term. The primary current surplus is projected to rise from 0.7 percent of GDP in 2003 to about 4 percent of GDP in 2008 (Table 5), while interest costs in percentage of GDP are projected to decline during the same period, contributing to debt sustainability. To achieve the primary current surplus target (and in line with the PRSP), the medium-term framework envisages an increase in the tax revenue-to-GDP ratio to 13 percent by 2008 .

36. Exports and imports will grow by an average rate of 16 percent and 12 percent per year, respectively, over the medium term (2005-08), spurred by trade liberalization and regional integration. Export growth will be fueled by diversification, both within and outside the EPZ, the improved quality of cotton, and a rebound in the volume of vanilla exports as measures are implemented to improve security and curb theft. As a result, the deficit in the external current account (excluding grants) should decline from 7.5 percent of GDP in 2003 to 6.9 percent in 2008.

\section{Program Monitoring}

37. The program will be monitored through semiannual reviews, as well as through the quantitative and structural performance criteria and benchmarks, indicative targets, and quarterly monitoring indicators shown in Table 14 and the MEFP (Appendix III, Attachment I, Tables 1 and 2). Several end-December quantitative performance criteria are likely not to have been met because of the fiscal slippages during the second half of 2003 . The staff therefore concurs with the authorities' requests that the next review be based instead on quantitative performance criteria for end-March 2004 and the PRGF arrangement be extended to March 1, 2005, in order to accommodate the final disbursement. The areas of technical assistance supported by the World Bank, the AfDB, and the EU are presented in Box 3. A revised technical memorandum of understanding (Appendix III, Attachment II) updates definitions and reporting requirements. The next semiannual review under the current arrangement is expected to be completed in August 2004. Relations with the Fund and the World Bank Group are summarized in Appendices IV and $V$, respectively. 


\section{Box. 3. Structural Conditionality}

\section{Coverage of structural conditionality in the program for 2004}

Structural performance criteria and benchmarks cover efficiency and transparency in the public sector, including completion of the bidding process for the purchase of the cotton company; completion of the bidding process for the selection of the company that will manage the sugar company; publication of the central bank's 2001, 2002, and subsequent financial statements and attendant audit opinions; and replacement of the current system of foreign exchange auctions with an interbank foreign exchange trading system.

\section{Structural areas supported by World Bank}

The World Bank is providing assistance to implement several structural reforms relevant to the Fund-supported program, including (i) assistance in strengthening the management of the electricity and water company, JIRAMA; (ii) reforms and capacity building at the land registry; (iii) launching of international tender for the withdrawal of the state from the cotton and sugar sectors; (iv) the strengthening of judicial procedures, and reform of the business law; (v) budget, treasury, and tax administration reforms; and (vi) strengthening of the monitoring of social expenditures. In the medium term, Bank assistance will gradually shift from investments to programmatic lending (Poverty Reduction Support Credits), requiring several policy actions fundamental to their success. A technical assistance project on governance to support the necessary structural reforms is under way.

\section{Structural areas supported by African Development Bank and European Union}

The African Development Bank proposes a new structural adjustment credit aimed at enhancing institutional capacity in the transport sector and supporting the fight against HIV/AIDS. The European Union's emergency budgetary assistance focuses on public expenditure management and social sector reforms.

\section{Structural measures covered by HIPC Initiative completion point conditionality}

The floating completion point conditionality includes the following measures relevant to the PRGF-supported program: (i) the strengthening of the budgetary control system through adoption of appropriate legislative changes and increased staffing; (ii) the establishment of a transparent information system for granting licenses in the mining, forestry, and fishing sectors, as well as biannual publication of the beneficiary list; and (iii) the recruitment of additional teachers for primary schools.

\section{TECHNICAL ASSISTANCE AND DATA ISSUES}

38. Madagascar has received extensive technical assistance from multilateral and bilateral donors over the past several years. Most recently, the Fund's technical assistance has focused on securing improvements in tax and customs administration, tax policy, public 
expenditure management and treasury accounting, international reserves management, the issuance of new currency, the central bank internal audit, banking supervision, anti-moneylaundering legislation, and balance of payments and monetary statistics (Appendix IV, Section XI). The authorities have recently requested Fund assistance for the development of the foreign exchange market, which will be provided in the coming months.

39. Madagascar's economic and financial statistics are comprehensive, but exhibit weaknesses with regard to the real sector, government finances, the balance of payments, and social indicators. These weaknesses do not, however, impede a meaningful assessment of economic policies or program monitoring. With technical assistance of the international community (including the Fund), the authorities are working to address these deficiencies and strengthen the statistical system.

\section{STAFF APPRAISAL}

40. Madagascar's macroeconomic performance in 2003, characterized by high growth and low inflation, indicates that economic recovery from the 2002 slump is ander way. The recovery remains fragile, however, and substantial effort is needed to maintain the growth momentum, ensure low inflation, and build an adequate cushion of international reserves. Moreover, while all the quantitative performance criteria for end-June 2003 were met, fiscal slippages emerged in the second half of the year and progress in implementing structural reforms has been slower than expected. Against this background, it is crucial that the authorities follow through on their commitment to implement a strong program for 2004 , including credible policies to strengthen the fiscal outlook and to accelerate structural reforms.

41. Based on timely implementation of the policies outlined in the MEFP, and assuming that foreign assistance flows materialize as projected, the staff considers the macroeconomic objectives for $\mathbf{2 0 0 4}$ to be feasible. These objectives have been set at realistic levels, recognizing that the higher growth and lower inflation rates achieved in 2003 represented recovery from the 2002 slump.

42. The stability in prices during 2003 is likely to have lowered inflation expectations. However, the lagged effect of the sharp expansion in domestic liquidity in the last quarter of 2003 could create inflationary pressures in 2004. To consolidate the 2003 disinflation gains, these effects should be contained. In this light, the staff is encouraged that the 2004 budget law requires a reduction in the government's net indebtedness to the banking system.

43. The fiscal stance for 2004 is in line with the macroeconomic objectives. The budget allows for modest increases in current expenditure, keeps the investment budget in line with the absorptive capacity of the economy and foreign financing, and aims at an ambitious, but feasible, revenue target. The staff welcomes the planned reduction in the wage bill relative to GDP and the increase in expenditure on education, health, and rural development. 
44. The staff is encouraged by the authorities' commitment to restore budgetary discipline, which was eroded in $\mathbf{2 0 0 3}$ when substantial extrabudgetary expenditure and domestic arrears were incurred. To avoid such slippages, it is imperative that the authorities at the highest level ensure that the provisions of the 2004 budget law are strictly observed.

45. Structural reforms in the fiscal area are critical. Weaknesses in budget preparation, expenditure control, and treasury operations should be addressed as soon as possible in order to avoid undermining budgetary control and prevent the accumulation of arrears. In particular, the irmplementation of the integrated budgetary system linking treasury and expenditure control operations needs to be accelerated.

46. The staff we]comes the measures included in the 2004 budget to improve the tax system, expand the tax base, and reduce external tariff barriers, while raising the tax revenue-to-GDP ratio. Achieving these objectives will require special efforts to strengthen tax administration, close loopholes that reduce collections, and recover tax arrears. As noted in the PRSP, government tax revenue should continue to rise over the medium term, so as to allow better delivery of social services and reduce dependence on foreign aid.

47. Ad hoc tax and tariff exemptions, such as those granted in August 2003, should be avoided as they undermine the effectiveness of the tax system. Even if they are meant to be temporary, these exemptions may prove politically difficult to remove or even to contain. The staff is encouraged by the commitment of the government not to renew the tax exemptions when they expire in 2005 and to avoid such measures in the future.

48. The monetary stance envisaged for 2004 is consistent with the growth, inflation, and balance of payments objectives of the program. To ensure satisfactory implementation of monetary policy, the central bank should strengthen its ability to forecast banking system liquidity. The conversion of long-standing government debt to the central bank into marketable securities is an important step in ensuring transparency in the financial relations between the government and the central bank. It should also pave the way for more active open market operations by the central bank. The staff welcomes the amendment of the Property Act allowing investors to own land, which, combined with an improved land registry system, should facilitate the use of land as collateral for bank loans. Emphasis should also be placed on developing the money market and expanding the operations of microfinance institutions.

49. Maintaining export competitiveness over the medium term is crucial. To reduce vulnerabilities and rebuild its export market share, Madagascar should accelerate structural reforms aimed at maintaining low production costs, improving the efficiency of the cotton sector, increasing the output and quality of the vanilla crop, and diversifying exports. Implementing prudent macroeconomic policies aiming at low inflation is also essential.

50. The privatization and rehabilitation of public enterprises are essential for improving the efficiency of the economy and enhancing export prospects. The staff is concerned that the delays in the implementation of structural reforms and privatization may 
erode the gains in economic efficiency, growth, and stability achieved in 2003. In particular, improving the efficiency of the utility company, privatizing the cotton company, and placing the sugar company under private management should be given high priority.

\section{The staff recommends the completion of the fourth review under the PRGF} arrangement, although short-term risks remain. These risks includc the possibility of policy reversals because of political reasons or resistance from vested interests affected by the policies. The attainment of the 2004 tax revenue target also depends largely on improvements in tax administration and collection of tax arrears, which may take time to realize. Moreover, exports may not grow as projected and the inflows of foreign assistance may not materialize as expected. If these risks were to materialize, the achievement of the program's objectives would require further fiscal adjustment. Against these risks should be set the broadly satisfactory implementation of the program until mid-2003, the strength of the authorities' 2004 program, including the steps being taken to combat corruption and improve governance, and the government's commilment to ensure budgetary discipline and take additional measures as needed. Accordingly, the staff recommends completion of the fourth review. The staff also recommends the extension of the PRGF arrangement until March 1, 2005 , the associated rephasing of disbursements, and provision of additional interim assistance under the HIPC Initiative. 
Figure 10. Madagascar: Change in Central Bank's Net Foreign Assets and Net Cumulative Purchase of Foreign Curreticy, January-December 2003

(In billions of Malagasy francs)

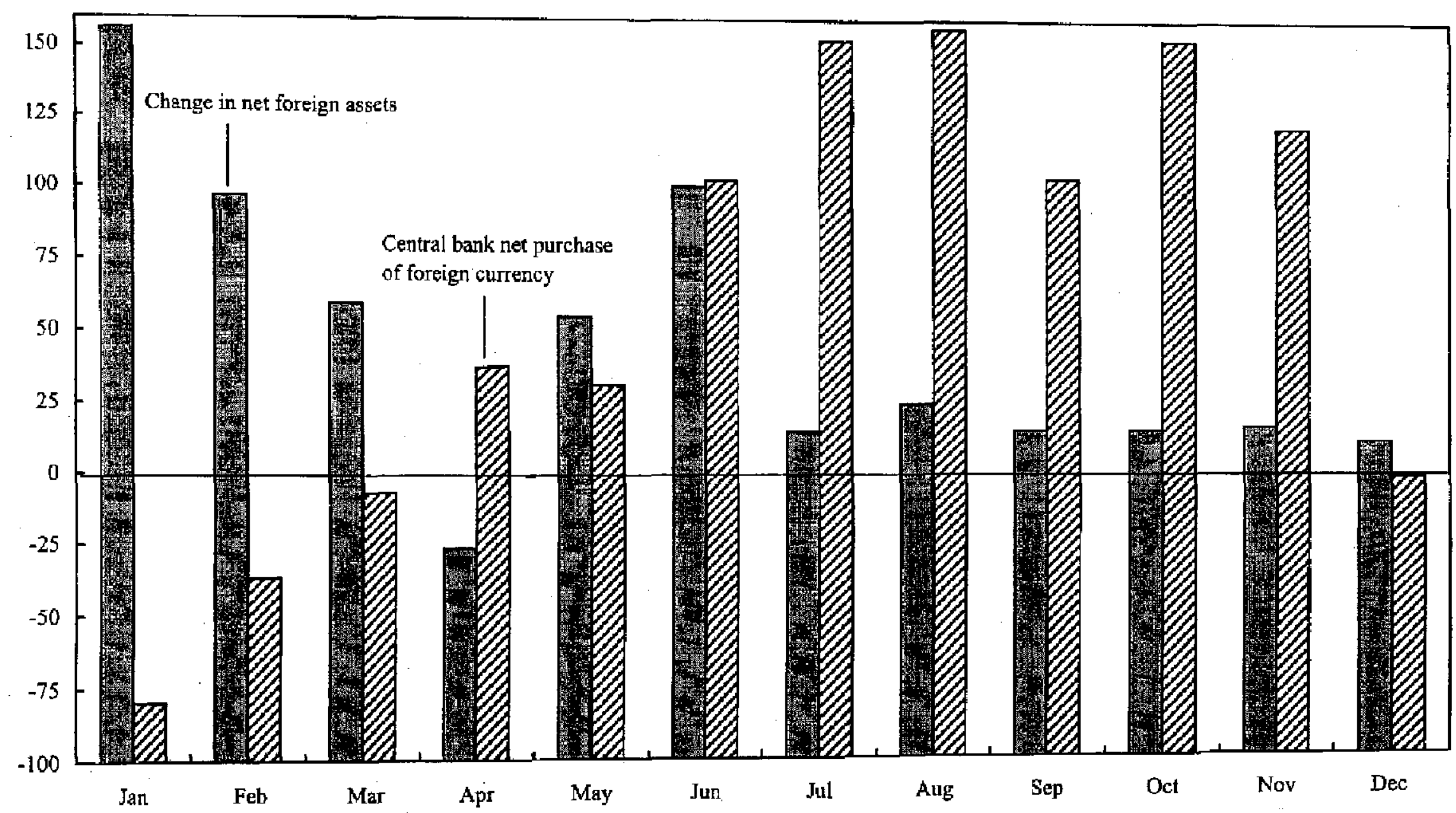

Source: Malagasy authorities 
Table 1. Madagascar: Tentative Work Program Under the Proposed Revised PRGF Arrangement, 2003-05

\begin{tabular}{lll}
\hline \multicolumn{1}{c}{ Date } & \multicolumn{1}{c}{ Action } & Disbursement \\
\hline October 2003 & $\begin{array}{l}\text { Mission to conduct fourth review under the } \\
\text { PRGF arrangement and discussion of } \\
\text { program for 2004. }\end{array}$ & SDR 11.347 million \\
March 2004 & $\begin{array}{l}\text { Executive Board consideration of the } \\
\text { fourth review under the PRGF } \\
\text { arrangement. }\end{array}$ & \\
May 2004 & $\begin{array}{l}\text { Mission to conduct the fifth review under } \\
\text { the PRGF arrangement. }\end{array}$ & \\
July 2004 & $\begin{array}{l}\text { Executive Board consideration of the fifth } \\
\text { review under the PRGF arrangement. }\end{array}$ & SDR 11.347 million \\
November 2004 & $\begin{array}{l}\text { Mission to conduct the sixth review under } \\
\text { the PRGF arrangement and discussions for } \\
\text { Article IV consultation. } \\
\text { February 2005 }\end{array}$ & $\begin{array}{l}\text { Executive Board consideration of sixth } \\
\text { review under PRGF arrangement, and } \\
\text { conclusion of the Article IV consultation. } \\
\text { Arrangement expires. }\end{array}$ \\
\hline
\end{tabular}


Table 2. Madagascar: Selected Ecomminic ana Finarcial Indicators, 20011-00

\begin{tabular}{|c|c|c|c|c|c|c|c|c|c|c|}
\hline & \multirow[t]{2}{*}{2001} & \multicolumn{2}{|c|}{2002} & \multicolumn{2}{|c|}{2003} & \multirow{2}{*}{$\begin{array}{l}2004 \\
\text { Rev. } \\
\text { Fog. }\end{array}$} & \multirow[t]{2}{*}{20005} & \multirow{2}{*}{\multicolumn{2}{|c|}{$\begin{array}{ll}2006 & 2(0) \\
\text { Projactions }\end{array}$}} & \multirow[t]{2}{*}{2000} \\
\hline & & Preg $^{2}$ & Esi. & Prug $^{2}$ & Proy. & & & & & \\
\hline & \multicolumn{10}{|c|}{ 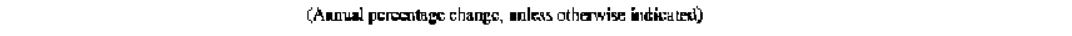 } \\
\hline 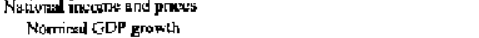 & 137 & 3.3 & 0.7 & 13.1 & 12.7 & 11.2 & 11.2 & 11.2 & 11.2 & 11.2 \\
\hline Real GDP growth & tiu & -11.9 & -127 & 6.0 & 9.6 & 6.0 & 6.0 & 8.0 & 6.9 & 6.0 \\
\hline GOP deElutur & 7.3 & 15.4 & 15.3 & 0.6 & 2.ä & 4.9 & 4.9 & 4.4 & 4.9 & 4.9 \\
\hline 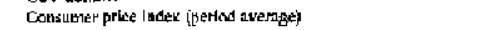 & T.4 & 15.8 & 15.8 & 8.0 & -1.4 & 3.0 & 5.0 & 90 & 5.8 & 5.0 \\
\hline Consuener pribe ladex (crut at periad) & 4.8 & 12.0 & 13.5 & 2.0 & 0.3 & 5.0 & $5 n$ & 3.0 & 5.0 & 5.0 \\
\hline \multicolumn{11}{|l|}{ External strilor } \\
\hline Expents, figstb & 206 & -46.5 & -90.5 & 56.0 & \$ll.4 & $\$ 4$ & 23.5 & 16.3 & $\mid 5.1$ & 10.0 \\
\hline Importy, n.i.t. & 5.5 & -33.4 & -37.6 & 45.0 & 313 & 16.7 & 18.9 & 125 & 9.5 & 7.4 \\
\hline Export viluing & 9.5 & -46.9 & $-51,6$ & 47.9 & 39.0 & 3.4 & 21.8 & 14.2 & [3.] & 8.1 \\
\hline lepsort volume & G.G & -32.9 & -4.5 .3 & 38.4 & 48.7 & 19.7 & 19.0 & 11.4 & y.] & 8.1 \\
\hline Terran of ticole (deteriocation -) & 11.2 & 1.6 & -10.4 & 0.7 & 6.7 & 7.8 & 1.5 & 29 & B.S & 2.4 \\
\hline Puffliv linante & & & & & & & & & & \\
\hline Revenue (Exolueding grants) & -1.3 & -25.7 & -20.7 & 30.3 & 45.5 & 28.4 & 14.3 & 15.0 & 15.6 & 14,1 \\
\hline Ioral expenuliture & 26.2 & -15.9 & -10.5 & 31.4 & 30.9 & 19.9 & 11.7 & 12.8 & 12.9 & 12.9 \\
\hline Current expendinure & 28.3 & 5.5 & 0.9 & 70,0 & 302 & 6.6 & 0.3 & 9.1 & 10.2 & 11.3 \\
\hline Invertmuar expenditurc and no kending & 23.5 & -37.1 & -2866 & 65.1 & 322 & 45.4 & 19.4 & 17.3 & 16.0 & 14.6 \\
\hline Maney antraredit" & & & & & & & & & & \\
\hline Broad mersey & 24.4 & 11.9 & 1.1 & 1.7 .4 & 12.0 & 12.0 & 14.9 & 14.5 & 14.5 & 14.2 \\
\hline Net koceigth nssets & 29,6 & 2.1 & -5.8 & $2 \pi .0$ & 15.0 & 33.1 & 2.0 & 34.0 & 26.9 & 22.0 \\
\hline Wher domextitc nssests & 2.0 & 9.3 & 13.0 & 8.6 & 10.9 & 2.9 & 21.0 & 60 & 76 & 9.1 \\
\hline Gradit to goncruical " & 31.0 & 9.1 & 31.2 & 17.0 & 21.0 & -4.7 & 0.0 & 0.6 & b.a & 0.0 \\
\hline Cedit the private seclor & 8.6 & 5.1 & .2 .6 & 37.6 & 18.3 & 20.7 & 21.9 & 21.2 & 22.7 & 23.2 \\
\hline Yelocity of money (MY3; avcrage) & 4.1 & 3.7 & 3.8 & 3.8 & 3.8 & 3.8 & 3.7 & 3.6. & 3.5 & 3.4 \\
\hline 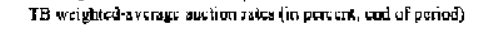 & $10 . n$ & $\cdots$ & 13.1 & $\cdots$ & $\cdots$ & $\ldots$ & - & $\cdots$ & .. & ... \\
\hline & & & & $(\operatorname{lop}$ & (GDP) & & & & & \\
\hline Pabs is finance & & & & & & & & & & \\
\hline Total reverue [axclwdiag granis) & 10.6 & 7.3 & 8.0 & 20.6 & 10.3 & 11.9 & 12.3 & 12.7 & 1.1.1. & 11,5 \\
\hline Of which: tane revene & 9.7 & 7.0 & 7.7 & 90,3 & 10,0 & I].2 & 11.6 & 12.1 & 126 & 13.0 \\
\hline Total yentb & 3.9 & 2.8 & 2.2 & 4.3 & 4.0 & 5.0 & 4.4 & 4.3 & 4.3 & 4.2 \\
\hline Capilal cxpendiure & 7,3 & 3.7 & 4.8 & 7.3 & 5.8 & 8.2 & 8.8 & 9.3 & 9.7 & 10,0 \\
\hline Totsil expenditure & 17.6 & 5.0 & 15.7 & 18.2 & 18.2 & 19.0 & 19.7 & 20.0 & 20.3 & 20.6 \\
\hline 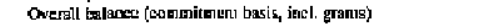 & -43 & 4.9 & .5 .5 & .33 & .3 .9 & -2.8 & -3.1 & -3.0 & -2.9 & -1.0 \\
\hline Overall tralance [commitment basis, cxel. grans] & -8.2 & -8.7 & -1.7 & -3.6 & -7.9 & -3.8 & -7.5 & -3.3 & -7.2 & -3.2 \\
\hline Dombatic financing & 1.9 & 2.2 & 2.4 & D.d & 2.1 & -0.1 & 0.0 & 0.1 & -0.2 & -0.1 \\
\hline Money and areditt ${ }^{2}$ & & & & & & & & & & \\
\hline Brodad tionty & 24,7 & 26.7 & 26.2 & 26.5 & 26.1 & 26.8 & 270 & 27.8 & 247 & 29.4 \\
\hline Nkt föreigrn nssets & 3.7 & B.I & 7.2 & 8.1 & $7, t$ & 9.0 & 82 & 9.9 & 11.3 & 124 \\
\hline 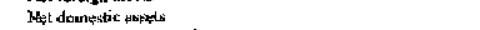 & 1E: 9 & 18.6 & 19.0 & 18.4 & 18.7 & IJ.3 & 18. 8 & 17.9 & 17.3 & $17.1)$ \\
\hline Credil to government & 6. & 8.6 & 9.0 & 9.3 & 9.7 & $\$ .3$ & 7.5 & 6.7 & s.0 & 5.4 \\
\hline Credit to the privale bector & 84 & 8.5 & $\$ .1$ & 8.4 & 8.5 & 9.2 & 10.0 & 11.0 & [2] & 13.4 \\
\hline Sautigys hnd investmem & & & & & & & & & & \\
\hline Resourse gip & -32 & -5.8 & tet & -7.6 & -7.9 & -8.5 & -8.2 & -8.1 & -7.8 & .7 .7 \\
\hline muestuent & ז8.5 & 11.1 & 14.3 & 17.5 & 16.0 & 19.4 & $20 \_5$ & 21.5 & 22.4 & 23.2 \\
\hline Govemonent & 7.3 & 3.7 & 4.8 & 7.1 & 5.8 & 8.2 & B.t & 9.3 & 9.7 & 10.0 \\
\hline Nengaverument & 11.2 & 7.3 & 9.5 & 10.2 & 10.2 & 11.2 & 11.7 & 12.2 & 12.7 & 13.2 \\
\hline Guss Jomestic suringe & 15.3 & 5.2 & 1.1 & 9.9 & 8. $\mathrm{L}$ & 10.9 & 123 & 13.4 & 14.6 & 15.5 \\
\hline Gross nationd sovings & 17.2 & 6.6 & 8.3 & 12.9 & $\mathrm{IL}, \mathrm{L}$ & 13.9 & 15.: & 16.1 & 17.1 & 17.9 \\
\hline Public & 3.0 & $-1,9$ & 0.7 & -3.3 & 1.5 & 5.4 & 3.7 & 6.3 & 6.8 & 7.1 \\
\hline Private & 14.2 & 8.5 & 9.0 & 16.2 & 9.6 & 8.5 & 9.4 & 9.8 & 50.3 & 10.8 \\
\hline 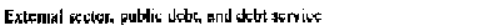 & & & & & & & & & & \\
\hline Export, tov, b & 21.3 & 11.7 & 11.0 & 16.0 & 14.5 & 14.5 & 16.7 & 18.1 & to 4 & 12.9 \\
\hline 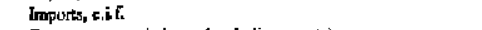 & 24.7 & 15,8 & 16.0 & 23.6 & 21.2 & 22.8 & $2 \$ n$ & 20.5 & 27.0 & 27,4 \\
\hline 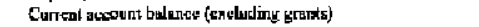 & -2.0 & -5.8 & -6.1 & -7.3 & -7.5 & -7.6 & -7.0 & -7.0 & -6.9 & -6.9 \\
\hline 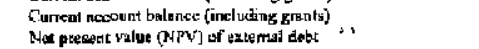 & -1.3 & $4, \overline{5}$ & -59 & -4.6 & -4.9 & $-5,4$ & -9.4 & -5.4 & -5.3 & -5.3 \\
\hline After tradütiunal dabt retief & 40.4 & 51.6 & 50,0 & 47.3 & 43.5 & 420 & 40.5 & 34.0 & 37.6 & 36.0 \\
\hline Aller echaneed HIPC Initialive debl relier & 45.9 & $\ldots$ & 3n. 7 & 29.8 & 27.3 & 27.1 & 26.7 & 26.3 & $35, y$ & 25.5 \\
\hline & & & & reent of $\mathrm{cx}$ & goads andil & (vices) & & & & \\
\hline Scbedaled extermal debt service " & & & & & & & & & & \\
\hline Afker tradítional dche relite & 10,6 & $\cdots$ & 11,9 & 11.8 & 120 & 12.8 & 10.1 & 9.6 & 3.6 & 8.0 \\
\hline Ailkr enhanted HIPC fnitiative debl relier & 3.9 & $\cdots$ & 4.7 & 5.0 & 5.1 & 7.0 & 5.6 & 5.3 & 47 & 1.4. \\
\hline & & & & $\ln n$ & (catcd) & & & & & \\
\hline 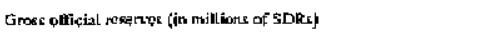 & 317.5 & 296.1 & 266.6 & 324.0 & $2 B 6.6$ & 4000. & 521.3 & 614.2 & $\operatorname{tos} .4$ & 793.0 \\
\hline In months of imparts of gonds and ronfictor services & 3.3 & 4.9 & 4.1 & 1.3 & 3.1 & 3.8 & 4.3 & 4.3 & 4.7 & 1.9 \\
\hline Exchauge rate; Malagasy finces per \$LKR (period averafe) & $8,397,4$ & $8,645,0$ & $8,774.9$ & $\ldots$ & $\ldots$ & $\ldots$ & $\ldots$ & $\ldots$ & $\ldots$ & $\ldots$ \\
\hline Excharge rate: Nalazkasy francs per U.S. dollar (period average) & 6.991 .5 & 5.680 .9 & 5.592.3 & $\ldots$ & $\ldots$ & $\ldots$ & $\ldots$ & $\ldots$ & $\ldots$ & ... \\
\hline GDP per capits (in U.S. dollars) & $2 \mathrm{BS}$ & 286 & 278 & 208 & 324 & 339 & 357 & 368 & 184 & 400 \\
\hline Nonninad GDP (billions of Malagary Imtics] & 29,843 & 30,847 & $30(2) / 2$ & 33.978 & 33.863 & 37,631 & 41,83 & 46,545 & 51,752 & 97.941 \\
\hline
\end{tabular}

Sourç̧: Malagasy sutharitics; and Fund staff crtimates and projections.

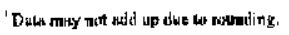

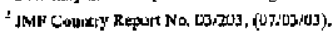

'Buscd on 1993 tradc weights.

2003 indusies extrabudgelery expenditure.

'The composition of monetary' grouth in 2003 reflecs reclassification of a public aterprisc, SOLIMA, debt (EMG 350.4 billian) by the goverunent.

'For 2003, inchude the tolkeover af SOLikA debt by the govenment.

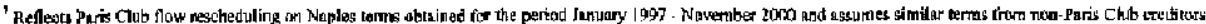

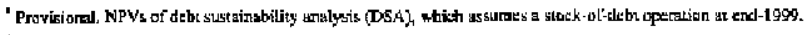

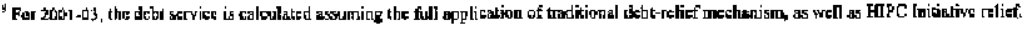

I" Encludiog gramls. 
Table 3. Mediegascar. Quintily Central Governement A ccoumb, 2003-04 in billions of Malagasy francs)

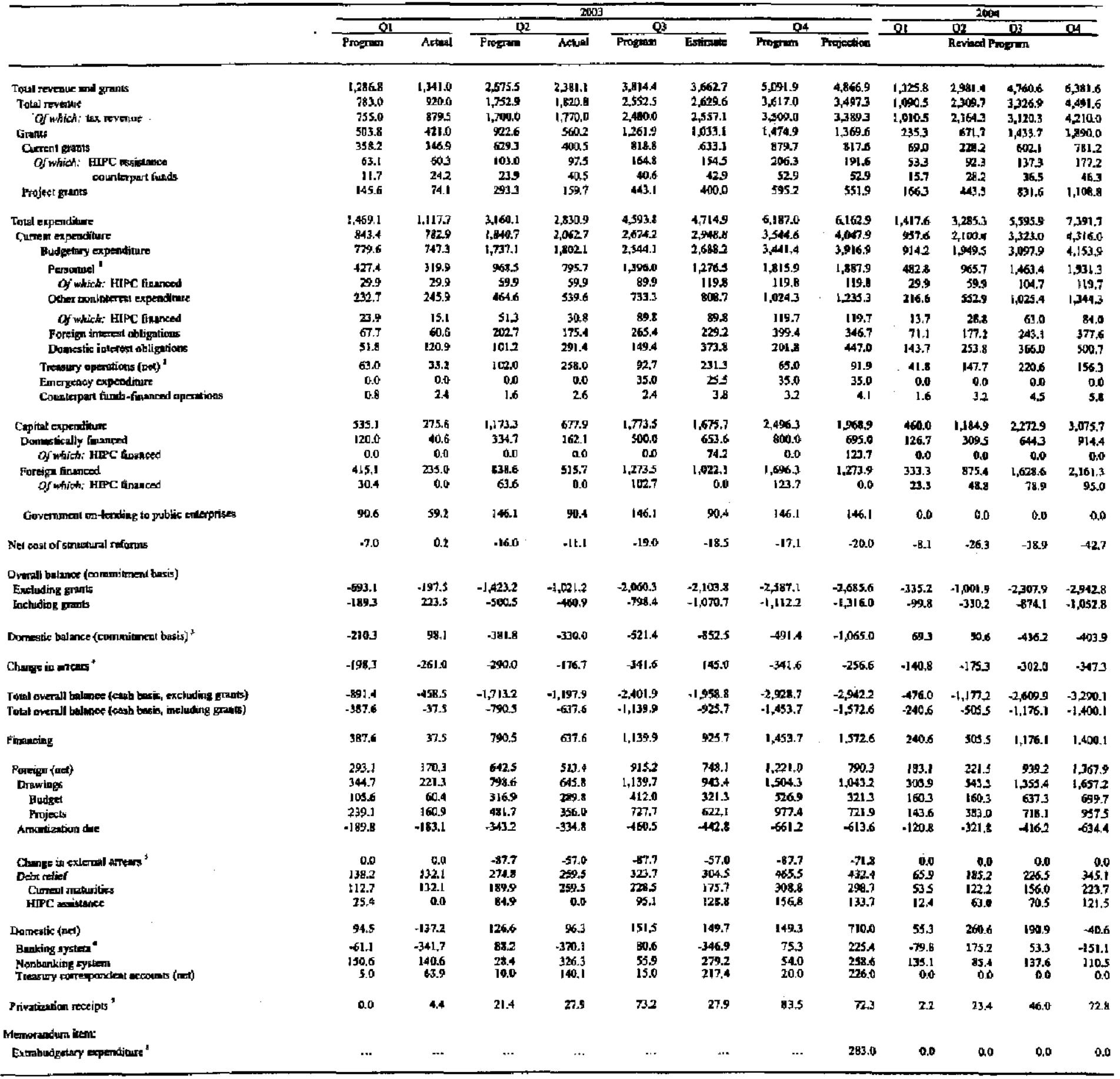

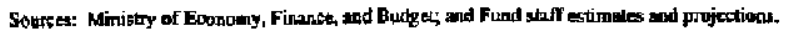

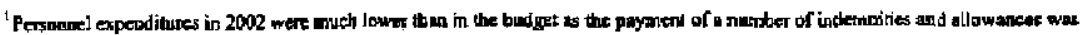
surpanded becinatit of the revenue shorifoll.

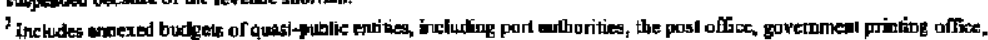

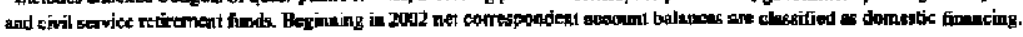

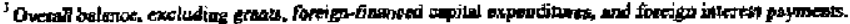

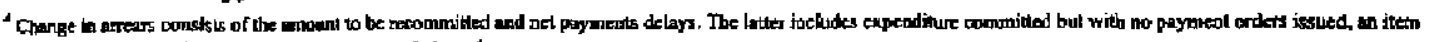

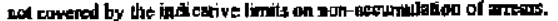

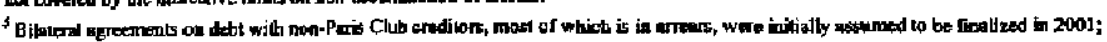

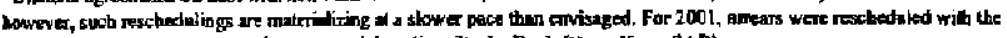

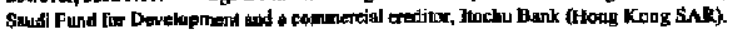

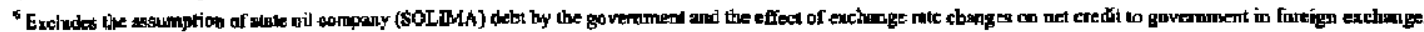

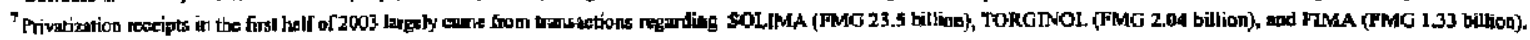

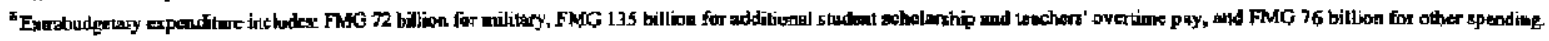




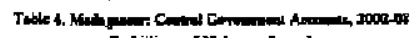

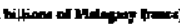

\begin{tabular}{|c|c|c|c|c|c|c|c|c|c|}
\hline & \multirow[t]{2}{*}{+00} & \multicolumn{2}{|c|}{2003} & \multicolumn{2}{|c|}{ Hitus } & \multirow[t]{2}{*}{$2 M 0$} & \multirow{2}{*}{$\frac{\text { atolo }}{\text { Prodectiont }}$} & \multirow[t]{2}{*}{2000} & \multirow[t]{2}{*}{ 20041 } \\
\hline & & mon. & Moj. & Fine & $\begin{array}{l}\text { Rer. } \\
\text { Prot. }\end{array}$ & & & & \\
\hline 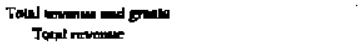 & $\begin{array}{l}3,053.1 \\
2,0030\end{array}$ & $\begin{array}{l}5,0915 \\
2,6172\end{array}$ & $\begin{array}{l}1,24,9 \\
3,497,3\end{array}$ & 5,4746 & $\begin{array}{l}6.5716 \\
4.491 .6\end{array}$ & 3.139 .9 & 5.915 .4 & $6,794.6$ & 10,172, \\
\hline Amagergy noveres & 20000 & intro & 3,4573 & 1,344, & 4.491 .6 & $5.135 \mathrm{~A}$ & $5,907.9$ & $5, \pi 40$ & 7.751 .1 \\
\hline Tex monstied. & 230012 & thathe & $3,514.3$ & $4,231.2$ & $\begin{array}{r}4,210.0 \\
\text { BDo.4 }\end{array}$ & 404.1 & \$.612. & 5520.7 & IAvOS \\
\hline 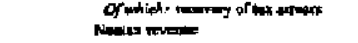 & sis & $\ddot{1050}$ & 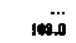 & 1133 & Itil. & $2 \pi 3$ & 275.5 & ב3.j. & m.9. \\
\hline$G_{\operatorname{man}}$ & 650.1 & $2,47.9$ & $1,3 \times 96$ & 1,5301 & 1, I90:a & 1003 & $2 ; 000.1$ & 20000 & $2,45.8$ \\
\hline Cune grous & 399.6 & 87m? & airs & 27 & 7012 & $12 t$ & an1. & nid & 00.2 \\
\hline 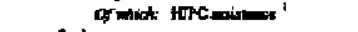 & 163.1 & sins & 101.6 & 0.0 & 137 & ao & 0. & $a_{t}$ & 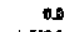 \\
\hline Pajon maxts & נon & 9952 & 9519 & wate & 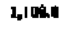 & 1,2172 & Ljk.1 & $1,+6687$ & $1,610.1$ \\
\hline Terat apostinger & $4, \pi 0,5$ & 6.1870 & 6,1529 & $6,91.3$ & 2,7917 & 4,259.] & $9,318.1$ & $10,512: 2$ & 11, w9.1.9. \\
\hline Annat mpentibure & $3,109,3$ & 0,5466 & 1,047s & $3,5.5$ & \$150 & 4, Saltat & $\sin 12$ & $5,513.1$ & $6,157.0$ \\
\hline 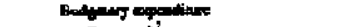 & IJI:E & 2,4414 & 29169 & $3,750,8$ & 4,1539 & $4,015,4$ & 4001,7 & 5,4101 & 8,0340 \\
\hline Finent" & 1,000 & 1,d15.9 & L,IITS & 1.5m.7 & 1.9313 & $2,091.1$ & 2234.2 & 20103 & $2,7004.4$ \\
\hline of wiveth: KRPC thearad & 71.4 & Ios & 1198 & 121.1 & 1197 & 146.] & เ57. . & 1620. & 171.4 \\
\hline 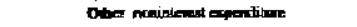 & $\boldsymbol{H + 1 . 7}$ & $1,024.3$ & :.235.3 & $1, \pi 3.1$ & 1,3403 & 1.520 .3 & $1,8090.6$ & $2,20.0$ & $2,5 m 3$ \\
\hline 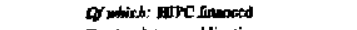 & נme, & 1097 & 189.3 & 12J., & $\mathbf{4 0}$ & Jiles & 110.7 & 1043 & 1202 \\
\hline 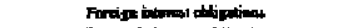 & 400,7 & 399. & $\mathbf{4 6 9}$ & 200.1 & $m, 6$ & 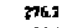 & 2769 & $2 x_{6,3}$ & 206.2 \\
\hline 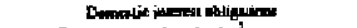 & 24.3 & 201.1 & $4+78$ & 200.9 & 5007 & 495.5 & Apes-s & $D_{-7} 7$ & 4han \\
\hline 7namyapatiom (ns) & 2020 & 15.0 & 91.9 & 4.0 & {$[5 \mathbf{6 1}$} & IOE,A & 1000 & 1020 & $1+m 0$ \\
\hline 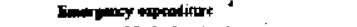 & 1772 & 3s.0 & 35.0 & 0.8 & $\mathbf{p . 0}$ & ext- & ad & $\infty 0$ & a.b \\
\hline 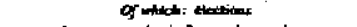 & 프구 & 15.0 & 55s.0 & 0.0 & ao & 0,0 & Q.D & 0.0 & $\mathbf{a . 0}$ \\
\hline 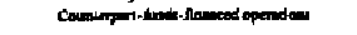 & 3.9 & 3.1 & $\$ .1$ & 3.0 & S.E & 1.0 & 30 & 1.0 & 3.0 \\
\hline Oppila expention & $1,45,4$ & $24+6.3$ & 1,409 & $3,113.0$ & $3,075 \%$ & $3,570.9$ & $4,314-3$ & $5,000,1$ & $\$ 776.2$ \\
\hline 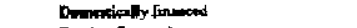 & 589.7 & $\mathbf{1 0 0 . 0}$ & 695.0 & $1,136.9$ & 94.4 & 1pol. & $1, \sec 1$ & $1,507.0$ & $2,321.4$ \\
\hline Forety finenoed & sith 2 & $1,696,3$ & 1,5738 & $15 \% .4$ & $2,161.3$ & $25: 9.3$ & 2.8112 & $3,006.1$ & $3,414.7$ \\
\hline 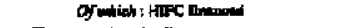 & 1313 & 123.7 & 123.7 & [203.5 & חהקו & +163 & as 2 & 129.3 & 196.0 \\
\hline 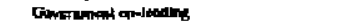 & 150.4 & 106.1 & 946.1 & 0.0 & 0.0 & a. & 0.0 & 0.0 & ose \\
\hline Fimby aurran halmaxs" & +6.3 & 6736 & 201.1 & $1,040.6$ & Ek2.4 & 1.165 .3 & 1,53999 & $1,930.6$ & $2,203.2$ \\
\hline \multicolumn{10}{|l|}{ 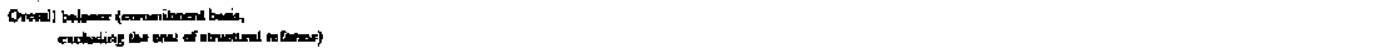 } \\
\hline 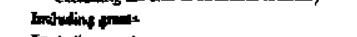 & A,, SOAt & $-1,005.1$ & $-1,21060$ & $-1,4,46.5$ & 1910.1910. & 1,7,7,7 & $-1,-1,28$ & $-1,5[11$ & $1,700,2$ \\
\hline Evecuting prents & $-2 . \mathrm{ns} s \mathrm{~s}$ & $-2,57000$ & $-2,455,6$ & $2,0,30.3$ & -200.1 & $-1,121.9$ & $.3,410.9$ & $-3,72.1$ &,+ 12010 \\
\hline 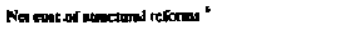 & 5.5 & .17 .1 & .200 & -10.9 & -2.7 & 0.0 & $\mathbf{0 . 0}$ & 0.0 & no \\
\hline Emocplonow reveare & 5.t & 10.0 & 10 & Wo: & 73 & 90 & 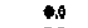 & 0.6 & 0.0 \\
\hline 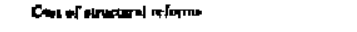 & 0 & 7.1 & 90.0 & s.1.0 & seco & 40 & 0.0 & 0,0 & A:D \\
\hline \multirow{2}{*}{\multicolumn{10}{|c|}{ 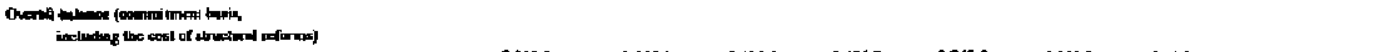 }} \\
\hline & & & & & & & & & \\
\hline 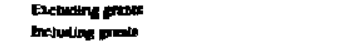 & $-2,3007$ & $-2,587.1$ & $2,41,6$ & -2000.7 & 2911.8 & $-3,1219$ & $-3,410,3$ & $-3,7,23.1$ & $-1,130,0$ \\
\hline 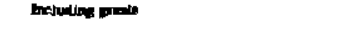 & -1.650 .6 & $-1 . t 12.1$ & $-1,5160$ & - I.this.6 & 1 1062. & $+6,294.7$ & $-1,462.5$ & -1,Jit. & 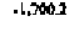 \\
\hline Dane lic belmese 7 & $-1,0068$ & $-\$ 1.4$ & $-1,40,0$ & -3751 & $-\infty 019$ & .258 .4 & -322.4 & 350.1 & $\rightarrow 290$ \\
\hline Chupen in aren & $-213,6$ & +341.6 & .2666 & 0.0 & $-3 T^{3}$ & -190.8 & -100.6 & -500 & -300 \\
\hline 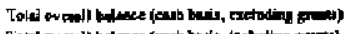 & $-2,5132$ & -2.921 .7 & $-2,91.2$ & $-1,606.7$ & $-3,391$ & .2739 & -3.510 .9 & $3 \pi m .1$ & $+1,17000$ \\
\hline 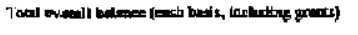 & $-1,16 \mathrm{~b}, 1$ & $-1,455.7$ & $-1,172.6$ & $-1,428,6$ & 1 & $-1,1,7,7$ & $-1,502.1$ & $-1,50.18$ & $-1,750.2$ \\
\hline Finencingy & 1.41.1 & $1,455.7$ & 1.97 .6 & 1,4586 & 1, , whel. & $1,44.9$ & 1.5012 .1 & 1,5 SAlB & 1,7303 \\
\hline Fonnigr $(t=1)$ & $1,4 \%, 2$ & 1.276 .0 & 7603 & 1,5786 & 1,2679 & $.1,47, \ldots$ & $1,534.0$ & $1,557,9$ & 1,40199 \\
\hline Daming & $1,150,1$ & $1,504.3$ & $1,+432$ & $1,250,8$ & 1,0572 & 1.5139 & Los13 & 18130 & 1.591 .0 \\
\hline thedpin & 6004 & 536.9 & 2113 & 2902 & $\sin$ & 2580 & 301.5 & J0.2 & 2⿰氵工, 3 \\
\hline Poojets & 435.7 & 97.4 & 7219 & $1,031.6$ & 9593 & 1,2519 & L.349.1 & $1,500.1$ & 1,861.7 \\
\hline Anentivition ber & .513 .1 & $\$ 12$ & $\$ 136$ & A378 & $-634,4$ & -41.: & $-\$ 11.1$ & -561.6 & FIth 7 \\
\hline 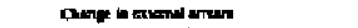 & DD & $-\$ 1.7$ & .71 .8 & 0,0 & $\$ 0$ & 0.0 & o. 0 & 0.0 & ao \\
\hline 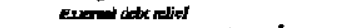 & $\$ 272$ & Ast & W12. & 360.7 & 345.1 & 365.6 & 3P1: & $\$ 06.5$ & $\$ 77.6$ \\
\hline 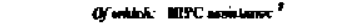 & 1600 & ISG.bB & 153.? & $\mathbf{1 0 . 7}$ & 1315 & 1656 & 393.: & $+0 \times 5$ & 427.6 \\
\hline Finercies of & 200 & 10 & 0.0 & 0.0 & $\$ 0$ & 0.0 & Da & 0.0 & 0.0 \\
\hline 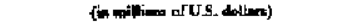 & 00 & 10 & 00 & 0.0 & $\$ .0$ & 60 & 0.0 & 0.0 & 0.0 \\
\hline Denember (mat) & 2218 & 249.3. & 7000 & 390,0 & 406 & -3.1 & -96.1 & $=08.1$ & $-52,7$ \\
\hline Bunking prosen" & 721 & 75.3 & 203.4 & Ima & .191.1 & $\$ 2.6$ & -90.6 & -129.6 & -100.4 \\
\hline 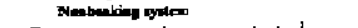 & .718 & 500 & 20.60 & $\operatorname{mon} .0$ & 114.3 & 79.9 & 60.4 & 36.3 & 54.7 \\
\hline 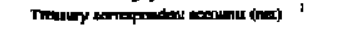 & 23 & 200 & 2760 & 0.0 & 10 & 0.0 & ש.0 & a.0 & $\mathbf{0 . 0}$ \\
\hline Privalizan ioe rexiples & 46.5 & 60.5 & 223 & 0.0 & 72.8 & 0.0 & $\mathbf{n , 0}$ & 0.0 & as \\
\hline Of intict; TEWh & $\cdots$ & $3+0$ & 0.0 & 0.0 & 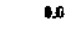 & 10 & e. & 0.0 & o. \\
\hline \multicolumn{10}{|l|}{ Momlonndem iver: } \\
\hline 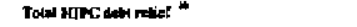 & 349.7 & 3632 & 32.3 & $\mathbf{3 6 3 . 7}$ & 290.7 & 365.6 & 393.: & 406.5 & 427.6 \\
\hline 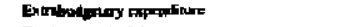 & & $\cdots$ & 283.0 & $\ldots$ & $\cdots$ & $\ldots$ & & $\because$ & :.: \\
\hline Tuled domsalic tad & 4.276 .4 & $\cdots$ & $4,720.4$ & $\cdots$ & 4,679, & 0.076 .7 & $9,049.6$ & 4.556.3 & 4.903 .8 \\
\hline
\end{tabular}

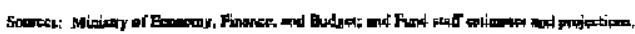

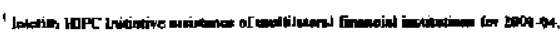

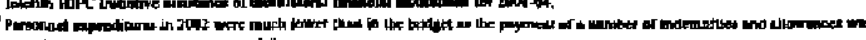

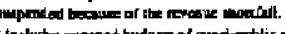

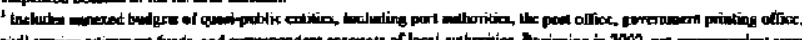

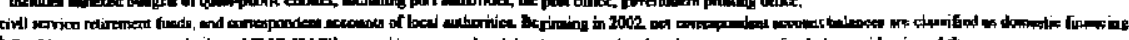

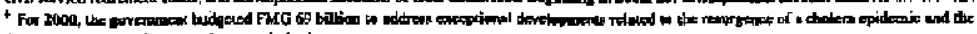

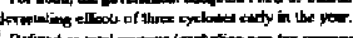

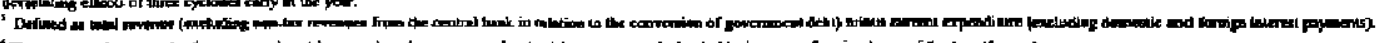

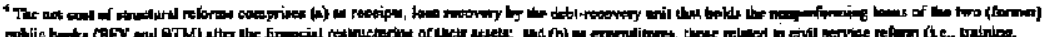

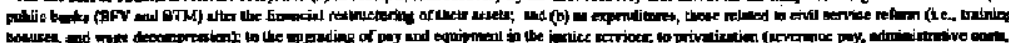

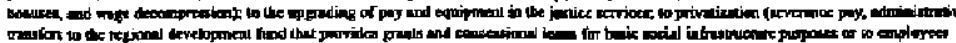

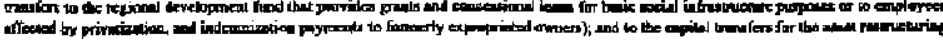

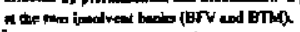

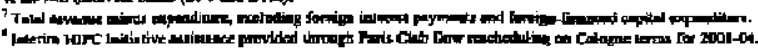

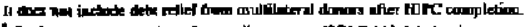

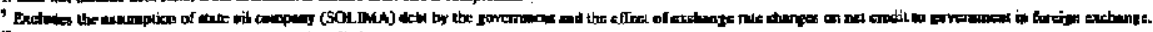

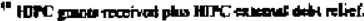




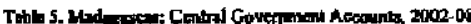

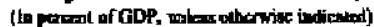

\begin{tabular}{|c|c|c|c|c|c|c|c|c|c|}
\hline & \multirow[t]{2}{*}{2002} & \multicolumn{2}{|c|}{2003} & \multicolumn{2}{|c|}{ 2004 } & \multirow[t]{2}{*}{2005} & \multirow{2}{*}{$\frac{2006}{\text { frojotion }}$} & \multirow[t]{2}{*}{2007} & \multirow[t]{2}{*}{2000} \\
\hline & & riog. & Proj. & Proge & $\begin{array}{l}\text { Rer. } \\
\text { Poge. }\end{array}$ & & & & \\
\hline Totol roverise and atinith & 10.2 & 15.0 & 1,4 & 14.5 & 165 & 16.6 & 17.0 & 17.4 & 17.7 \\
\hline Tolal nevernot & 8.0 & 10.6 & 10.3 & 11.5 & 115 & 12.3 & 12.7 & I3.1 & 135 \\
\hline Tux reveruse (whthout werovesy of the wrems) & 7.7 & 10.3 & 10.0 & 11.2 & 11.0 & 11.6 & 12.1 & 12.6 & L13.0 \\
\hline Reourvary of tha motess & $\cdots$ & $\ldots$ & $\ldots$ & $\ldots$ & 0.2 & $\ldots$ & $\ldots$ & ... & $\ldots$ \\
\hline Notax revenue & 0.3 & 0.3 & 0.3 & 0.3 & 0.7 & 0.7 & 9.6 & D.s & e.5 \\
\hline Orants & 2.2 & 4.3 & 4,0 & 3.0 & 3.0 & 4.4 & 4,3 & 4.3 & 4.1 \\
\hline Cursol govil & $0, \hat{4}$ & 2.6 & 2.4 & $\mathbf{0 . B}$ & 21 & 1.5 & I.4 & 1.4 & 1.4 \\
\hline Or which: HIFC mosipentere" & 0.6 & 0.0 & 0.6 & 0.0 & 0.5 & 0.0 & 0.0 & 0.0 & 0.0 \\
\hline Projert grents & 1.3 & 1.8 & 1.6 & 22 & 2.8 & 2.9 & 2.9 & 28 & 2.8 \\
\hline Total experititures & 15.7 & 15.2 & |B.2 & IR, 3 & 15.6 & 19.7 & 20.0 & $\mathbf{2 0 . 3}$ & 20.6 \\
\hline Curnent apaditure & 10.3 & 10.4 & 120 & 10.1 & 115 & 11.0 & Ia.8 & $\mathbf{N C . 7}$ & 10.3 \\
\hline Noqintetsil apent|ruse & 3.1 & ad & 9.2 & 8.6 & 8.7 & 8.9 & B.9. & 9.0 & 9.2 \\
\hline Pastones & 4.6 & 53 & 5.6 & 5.1 & $\$ .1$ & 5.0 & 4.8 & 4.7 & 4.7 \\
\hline Of whish:HIPC finotwond & 02 & 0,4 & a.6 & 0.0 & 0.3 & 0.3 & 0.3 & 0.3 & 0.3 \\
\hline Ohro nomintenest expentiture & 2.5 & 3.0 & 3.6 & 3.5 & 36 & 3.9 & 4.1 & 43 & 4.5 \\
\hline of which;HIPC finenced & 0.4 & a.d & 0.4 & 0.0 & 0.2 & 0.2 & 0.2 & 0.2 & 0.3 \\
\hline Interest obligutiens, & 2.2 & 1.3 & 2.3 & 1.3 & 23 & 1.8 & 1.7 & 1.5 & 1.3 \\
\hline Trassang operations ${ }^{2}$ & 0.7 & 0.2 & 0.3 & 0.1 & 0.4 & 02 & 0.2 & 02 & 0.2 \\
\hline 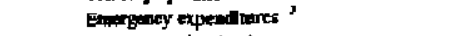 & 0.4 & 0.1 & 0.1 & 0.0 & 0.0 & na & 0.0 & 0.0 & 0.0 \\
\hline or which sloctions & 0.2 & 0.1 & $0 . t$ & 0,0 & 0.0 & 0.0 & 0.0 & 0.0 & 0.0 \\
\hline Caphrol expendibure & 4.8 & 7.3 & 5.8 & 8.2 & 8.2 & 8.8 & 9.3 & 9.7 & 10.0 \\
\hline Domertionlly frumeed expenctiture & 1.9 & 2.3 & 2.1 & 3.0 & 2.4 & 2.6 & 3.2 & 3.7 & 4,0 \\
\hline Forcifo-liwithod orpenditurs & 2.9 & 5.0 & 3.3 & 52 & 5.7 & 6.2 & 6.0 & 60 & 3.9 \\
\hline Of whict HLPC finuoced & 0.4 & 0.4 & 0.4 & A.3. & 0.3 & 0.3 & 0.3 & 01 & 0.2 \\
\hline Govermment on-lending & 0.5 & 0.4 & 0.4 & D.D & 0.0 & B.D & 0.0 & $\mathbf{\infty}$ & $\mathbf{0 , 0}$ \\
\hline Donfatic budgaty balones & -2.9 & -1.0 & -2.7 & $-0,8$ & -1.0 & -0.6 & -0.7 & -0.7 & -0.7 \\
\hline Primbly cuerent balawer & $-a .2$ & 2.0 & 0.7 & 28 & 2.3 & 28 & 3.3 & 3.3 & tis \\
\hline Nel cost of frtindanl reforms " & 0.0 & -0.1 & $-0,1$ & -0.1 & 由.1 & 0.0 & 0.0 & 0.0 & 0.0 \\
\hline Escoptional revenut & 0,0 & 0.0 & 0.0 & 0.0 & 0.0 & 0.0 & 0.0 & 0.0 & 0.0 \\
\hline Cost of somentral reforms & a.d & 0.1 & 0.1 & 0.I & 0.1 & 0.0 & 0.0 & o.j & 0.0 \\
\hline \multicolumn{10}{|l|}{ 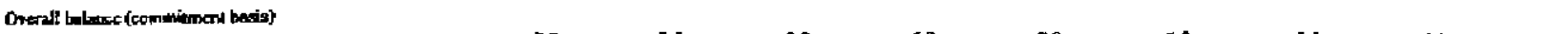 } \\
\hline Exchuditing frants & -7.7 & -7.6 & -7.9 & -6.9 & -7.8 & -7.5 & -7.3 & -7.2 & .72 \\
\hline Inchudieg granls & -5.5 & -1.3 & -3.9 & -3.9 & -2.8 & -3.1 & -3.0 & -7.9 & -3.0 \\
\hline Dosmenstic belimite & -3.4 & .1 .4 & +3.1 & -0.9 & -1.1 & -0.6 & -0.7 & -4.7 & -0.7 \\
\hline Change in arroms & -0.7 & $\cdot 1,0$ & -0. & 0.0 & -0.9 & -0.4 & $-0,2$ & $-\$ .1$ & -0.1 \\
\hline 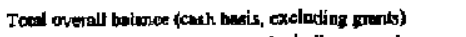 & -8.4 & .8 .6 & -8.7 & -6.9 & -8.7 & .7 .8 & -75 & .73 & -7.2 \\
\hline 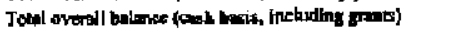 & -6.2 & 4.3 & 4.6 & .3 .9 & +3.7 & -3.3 & -3.1 & $-5,6$ & -3.0 \\
\hline Fimunchang & 6.2 & 4.3 & 4.6 & 3.9 & 3.7 & 3.9 & 3.2 & $\mathbf{1 . 0}$ & 3.0 \\
\hline Fontign (nt1) & 3.6 & 3.6 & 2.3 & 3.1 & 3.6 & 33 & 35 & 3.2 & 3.1 \\
\hline Drawings & 3,8 & 4.4 & 5,1 & 3.3 & 4,4 & 3.6 & 3.5 & 3.s & 3,5 \\
\hline Eudget & 2.2 & 1.6 & 6.9 & 0,6 & 1.9 & 0.6 & 0.6 & 0.6 & 0.6 \\
\hline Projestis & 1.6 & 2.9 & 2.1 & 27 & 2.5 & 3.0 & 2.9 & 2.9 & 23 \\
\hline Amortizetion dus & -1.9 & +1.9 & -3.3 & -1.2 & -1.7 & -1.0 & $-1,2$ & -1.1 & -1.1 \\
\hline Change in exictial armers & 9.0 & -0.3 & -0.2 & a.o & 0.0 & 0.0 & 0.0 & 0.0 & 0.0 \\
\hline Kicteringl debt retiter & 1.8 & 1.4 & 1.3 & 1.0 & 0.9 & 0.9 & 0.8 & O.E & 0.7 \\
\hline Of which: HIPC anifence" & 0.6 & 0.5 & 0.4 & 1.0 & 0.3 & 0,9 & a.t. & D.:B & 0.7 \\
\hline Finencing Ep & 9.0 & 0.0 & 0.0 & 0.0 & 0.0 & 0.0 & 9.0 & 0.0 & 0.0 \\
\hline Dumestic (nox) & 2.4 & 0.4 & 2.1 & 0.8 & -0.1 & 0.0 & -0.1 & -0.2 & -0.1 \\
\hline of which: balloing pystem ${ }^{7}$ & 2.4 & 0.2 & 0.7 & 0.3 & -8.4 & .0 .2 & $\$ .2$ & -0.2 & -0.2 \\
\hline P tivahiration receipt: & 0.2 & $\sqrt{2}$ & 0.2 & no & 0.2 & 0.0 & 0.0 & 0.0 & 0.0 \\
\hline \multicolumn{10}{|l|}{ Momponanduat jkems: } \\
\hline Totnd HIPC deh retie! & 1.2 & 1.1 & 1.0 & 1.0 & 0.8 & 0,9 & $0, \mathbf{3}$ & 0.8 & 0.7 \\
\hline Echuogation repentiume, wfirs HIPC & 2.8 & $\cdots$ & $\cdots$ & $\ldots$ &.+ & $\ldots$ & $\cdots$ & $\ldots$ & $\ldots$ \\
\hline 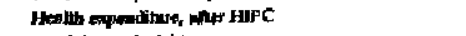 & 0.9 & $\ldots$ & $\ldots$ & $\cdots$ & $\ldots$ & ... & $\ldots$ & $\ldots$ & $m$ \\
\hline Total dowantic debt & 14.1 & $\ldots$ & 12.9 & $\ldots$ & 12.4 & 11.2 & 10,0 & 8.8 & 7.8 \\
\hline Nonsinal GDP (in billiona of Malagery fidsis) & · 30,042 & 33,978 & 33,163 & 37,779 & 97,651 & 41,963 & $46,5-15$ & 51,752 & 57,541 \\
\hline
\end{tabular}

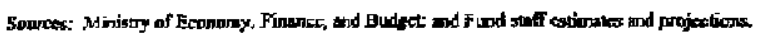

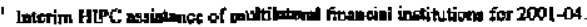

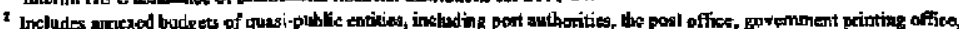

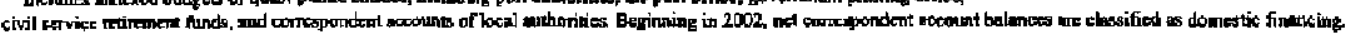

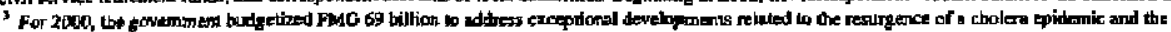

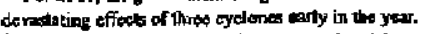

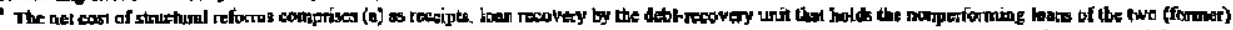

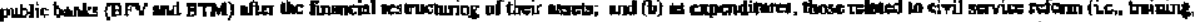

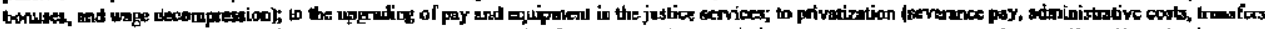

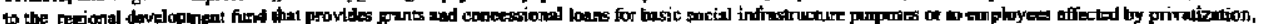

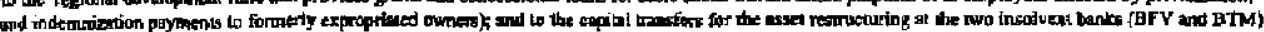

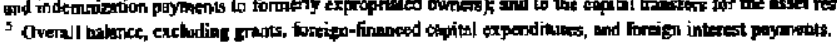

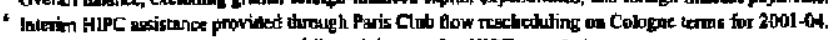

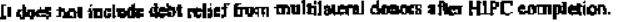

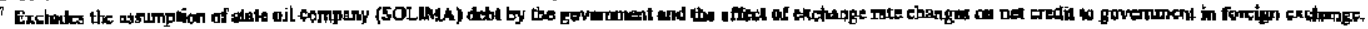


Table 6. Madagascar: Monetary Surver, 2002-14

(In billions of Malagesy france, upiess atherwise indicated)

\begin{tabular}{|c|c|c|c|c|c|c|c|c|c|c|c|c|c|}
\hline & \multirow[t]{3}{*}{2002} & \multicolumn{8}{|c|}{2003} & \multicolumn{4}{|c|}{2004} \\
\hline & & \multicolumn{2}{|c|}{ Mar. } & \multicolumn{2}{|c|}{$\mathrm{Jum}}$. & \multicolumn{2}{|c|}{ Sep. } & \multicolumn{2}{|c|}{ Dec- } & Mar. & Jun. & Sep. & Des. \\
\hline & & Proj. & Act. & Prog. & Aat & Prog. & Est. & Prog. & Proj. & \multicolumn{4}{|c|}{ Revised Program } \\
\hline Net foreign ussets & $2,172.3$ & $2,398.7$ & $2,300.9$ & $2,378.9$ & $2,422.7$ & $2,531,4$ & $2,457,9$ & $2,737.0$ & $2,498,4$ & $2,726.5$ & $2,585.6$ & $3,446,4$ & $3,374.5$ \\
\hline Net foreign assets (BCM) & $1,286.1$ & $1,382.8$ & $1,345,5$ & $1,270.0$ & $1,336.8$ & $1,360.0$ & $1,475.2$ & $1,773.1$ & $1,451.6$ & $1,673.4$ & $1,532.5$ & $2,393.3$ & $2,321.5$ \\
\hline Net forrign assets (domestic money banks) & 886.1 & $1,015.8$ & 955.4 & $1,108.9$ & $1,03,5,9$ & 1.171 .4 & 982.6 & 963.9 & $1,046.8$ & 1.053 .1 & $1,053.1$ & $1,053.1$ & $1,053.1$ \\
\hline Net domestic assets & $5,704.8$ & $5,847,7$ & $5,664.3$ & $6,159.1$ & $5,224.3$ & $6,218.8$ & $5,723.4$ & $6,259.0$ & $6,323.9$ & $6,713.4$ & $7,074,8$ & $6,346,3$ & $6,506.4$ \\
\hline Domes tio oredit & $5,952.4$ & 6.135 .1 & $5,809.3$ & $6,398.4$ & $5,552.7$ & $6,484.0$ & $5,878.6$ & $6,553.2$ & 6,5690 & $6,690.7$ & $7,076.6$ & $7,128.1$ & 7.012 .5 \\
\hline Net credil to gaverthent (bodget) $\mathrm{v}$ & $2,706.8$ & 2661.4 & $2,363.1$ & $3,168.0$ & $2,316.3$ & $3,166.4$ & $2,700.6$ & $3,167.6$ & $3,274,9$ & $3,193.1$ & 3.448 .1 & $3,326.2$ & $3,121.8$ \\
\hline Dther clains on public sector & 405.4 & 461.3 & $4 \mathrm{I} 6.2$ & 461.3 & 382.0 & 461.3 & 368.8 & 461.3 & 368.8 & 368.8 & 368.8 & 368.8 & 368.8 \\
\hline Crodit to the economy & $2,840.3$ & $3,010.3$ & $3,030.1$ & $2,769.1$ & $2,854.3$ & $2,856.2$ & $2,809.2$ & $2,924,3$ & $2,925,3$ & $3,128.7$ & $3,259.7$ & $3,433.2$ & $3,521.9$ \\
\hline Credit to public entetprises if & 405.8 & 405.3 & 409.6 & 62.5 & 391.1 & 62.5 & 44.7 & 62.5 & 4.7 & 44.7 & 44.7 & 44.7 & 44.7 \\
\hline Credit to private seotor & $2,494.5$ & $2,605,1$ & $2,620.5$ & $2,706.6$ & $2,463.3$ & $2,793,8$ & $2,764.5$ & 2.861 .9 & $2,880.5$ & $3,084.0$ & $3,214.9$ & $3,388.4$ & $3,477.1$ \\
\hline Other iterbs (tret; asset +) & -247.6 & -287.4 & -145.1 & -239.3 & .328 .3 & -265.2 & -155.2 & $-294,2$ & -245.1 & 22.7 & -1.8 & .781 .8 & -506.1 \\
\hline M3 & $7,877.0$ & $8,246.3$ & $7,965,2$ & $8,538.0$ & $7,647.0$ & $8,750.3$ & $8,181.3$ & $8,996,0$ & $8,822.3$ & $9,439.9$ & $9,660.4$ & $9,792.7$ & $9,881.0$ \\
\hline Foreign crovency deposits & $B 78.9$ &, 059.1 & 1,028.0 & $1,028.0$ & 995.9 & $1,028.0$ & $1,014.6$ & $1,028.0$ & $1,030.0$ & $1,036.2$ & $1,036.2$ & $1,036.2$ & $1,096.2$ \\
\hline M2 & $6,998,2$ & $7,187,2$ & $6,937.2$ & $7,510.0$ & $6,651.1$ & $7,722.3$ & 7.166 .7 & 7,9680 & 7.792 .3 & B, 403.6 & $8,624.2$ & 8.756 .5 & $8,844.8$ \\
\hline Chureney in circulation & $2,330 . \mathrm{L}$ & $2,407.9$ & $2,319,4$ & $2,527.2$ & $2,153.4$ & $2,590.1$ & $2,543.0$ & $2,698.8$ & $2,646.7$ & 2,832.0 & $2,898.1$ & $2,937.8$ & $2,964.3$ \\
\hline Deposits in lecal currency 2 ! & $4,490,7$ & $4,603.0$ & $4,434.2$ & $4,799.2$ & $4,330.4$ & $4,948.6$ & $4,454.1$ & $5,085.7$ & $4,975.9$ & $5,402.0$ & $5,556.4$ & $5,649,0$ & $5,710.8$ \\
\hline Shortuterth obligations of conmercial banks & 177.4 & 176.2 & 183.5 & 183.5 & 167,3 & 189.5 & 169.7 & 183.5 & 169.7 & 169.7 & 169.7 & 169.7 & 169.7 \\
\hline Memorandum iterns: & & & & (Paces & change sin & he and of $t$ & pievious y & unless of & wise indise & & & & \\
\hline M3 & 7.1 & 4.0 & l.1 & 7.6 & -2.9 & 10,3 & 3.9 & 13.4 & 120 & 7.0 & 9.5 & 11.0 & 12.0 \\
\hline M2 & 6.3 & 2.7 & -0.9 & 6.5 & -5.0 & 9.5 & 2.4 & 13.0 & 11,3 & 7.8 & 10.7 & 12.4 & 13.5 \\
\hline Domestic credit & 12.2 & 3.1 & $-2,4$ & 6,5 & -6.7 & 3.9 & -1.2 & 9.1 & 10.4 & 1.9 & 7.7 & 8.5 & 6.8 \\
\hline Credit to the private sector & -2.6 & 7.0 & 7.6 & 11.2 & 1.2 & 14.8 & 13.6 & 17.6 & 18.3 & 7.1 & 11.6 & 17.6 & 20.7 \\
\hline Wet credit to govemunent $/ /$ & 32.2 & -1.5 & -12.7 & 17.0 & -14.4 & 10.0 & -0.2 & 17.0 & 21.0 & -2.5 & 5.3 & 1.6 & -4.7 \\
\hline Reserve money & 4.4 & -6.7 & -13.1 & .7 .4 & -14.2 & -6.7 & 4.8 & -4.7 & 3.9 & 6.4 & 8.7 & 10.1 & 11.0 \\
\hline Cumency/M3 (in percent) & 29.6 & 29.2 & 29.1 & 29.6 & 28.2 & 29.6 & 31.1 & 30.0 & 30.0 & 30.0 & 30.0 & 30.0 & 30.0 \\
\hline Reserve money multiplier (M3/resarves) & 2.2 & 2.5 & 2.6 & 2.6 & 2.5 & 2.7 & 2.3 & 2.7 & 2.4 & 2.4 & 24 & 2.4 & 2.4 \\
\hline Veloclty of money (GDP/end-of-period M3) & 3.8 & $\cdot \ldots$ & $\ldots$ & $\ldots$ & $\ldots$ & ... & $\ldots$ & 3,8 & 3.8 & .. & $\ldots$ & $\ldots$ & 3.8 \\
\hline Exchange rate (Malagosy frances pe SDR; end of period) & $8,746,0$ & $9,108.0$ & $8,848,4$ & $\$, 240.5$ & $8,557.3$ & $9,371.6$ & $8,719.1$ & $\ldots$ & $\ldots$ & $\cdots$ & $\ldots$ & $\cdots$ & $\cdots$ \\
\hline
\end{tabular}

Sources: Ceutral Bank of Madagasear $(B C M)_{1}$ and Fund stuf ertimates and projeclions.

1/2003 betodes the takeover of atate cil company (SOLCMA) debt (FMG 350.4 billion) by the goreuriment

$2 /$ Includes Comples de Cheques Fostaux and Caisse d'Épargme de Madagascar deposit accounts. 
Table 7. Madinghscar: Balnnce Stheet of the Central Bank urid Consolidated Balauce Sheet of Commercial Bank8, 2002-04

(In billions of Malagagy franes, uniess otherwige indicatedh)

\begin{tabular}{|c|c|c|c|c|c|c|c|c|c|c|c|c|c|}
\hline & \multirow[t]{3}{*}{2002} & \multirow{2}{*}{\multicolumn{6}{|c|}{2003}} & \multirow{2}{*}{\multicolumn{2}{|c|}{ Dec. }} & \multicolumn{4}{|c|}{2004} \\
\hline & & & & & & & & & & Mar. & Jü. & Sep. & Dec. \\
\hline & & \multicolumn{2}{|c|}{ Proj. } & Prog. & Act. & Frog. & $\overline{\text { Est. }}$ & \multicolumn{2}{|c|}{ Prog. } & \multicolumn{4}{|c|}{ Reviged Program } \\
\hline \multicolumn{14}{|l|}{ Central hank (BCM) } \\
\hline Net foreign ssgets & $1,286.1$ & $1,382,8$ & $1,345.5$ & $1,270.0$ & $1,386,8$ & $1,360,0$ & $1,475,2$ & $1,773.1$ & $1,45] .6$ & $1,673.4$ & $\mathbf{1}, 532.5$ & $2,393,3$ & $2,321.5$ \\
\hline Net domestip sssets & $2,248.9$ & $1,913.9$ & $1,725.7$ & $2,002.0$ & $1,645.5$ & $1,938.6$ & $2,032.6$ & $1,596.9$ & 2.222 .4 & $2,236.9$ & 2.462 .4 & $1,652.4$ & $1,758.1$ \\
\hline Oxernll atredil to govemnent (net) & $1,585.2$ & $1,080,1$ & 884.0 & $1,588.4$ & 906.3 & $1,550.8$ & $1,356,4$ & $1,237,9$ & $1,817.2$ & $1,713.2$ & $1,915.2$ & $1,935.2$ & $1,715,2$ \\
\hline Liquidity operations If & 0.0 & 0.4 & 0.0 & .369 .8 & 0.0 & -413.5 & 0.0 & -732.8 & 0.0 & 0.0 & 0.0 & 0.0 & 0.0 \\
\hline Net credit to govermment (budget) $2 /$ & $1,585.2$ & $1,079.7$ & 884.0 & $1,958.2$ & 906,3 & $1,964,2$ & $1,356.4$ & $1,970.7$ & $1,817.2$ & $1,715.2$ & $1,915.2$ & $1,935.2$ & $1,715.2$ \\
\hline Claims on public enterprises $2 /$ & 364.1 & 368.3 & 371.2 & 20.8 & 362.5 & 20.8 & 17.8 & 20.8 & 17.8 & 17.8 & 17.8 & 17.8 & 17.8 \\
\hline Credil to banks & 27.2 & 27.2 & 26.5 & 26.5 & 22.1 & 26.5 & 21.3 & 26.5 & 21.3 & 21.3 & 21.3 & 21.3 & 21.3 \\
\hline Other items (net, asset +) & 272.3 & 438.3 & 444.0 & 366.3 & 354,6 & 340,6 & 637.1 & 311.6 & 366.1 & 482.5 & 508.8 & .322 .0 & 3.8 \\
\hline Of which : valuation account (losses -) & 54.5 & 98.1 & 20.9 & 77.6 & 77.1 & -103.4 & 76.5 & -132.3 & 52.5 & 43.6 & 43.6 & 43.6 & 43.6 \\
\hline Resterve money & $3,535.0$ & $3,296.7$ & $3,071.2$ & $3,272.0$ & $3,032.3$ & $3,298.6$ & $3,507.8$ & $3,370.0$ & $3,674.0$ & $3,910.3$ & $3,994.9$ & $4,045.7$ & $4,079.6$ \\
\hline Currency outside banks & $2,330.1$ & $2,407,9$ & $2,319.4$ & $2,527.2$ & $2,153,4$ & $2,590.1$ & $2,543.0$ & $2,698.8$ & $2,646.7$ & $2,832.0$ & $2,898.1$ & $2,937.8$ & $2,964.3$ \\
\hline Bank reserves & $1,204,9$ & 888.8 & 751.8 & 744.8 & 878.9 & 708.5 & 964.8 & 671.2 & $1,027.3$ & $1,078.3$ & $1,096,8$ & $1,107.9$ & $1,115.3$ \\
\hline Currency in banks & 122.6 & {$[22.6$} & 102.3 & 102.3 & 1375 & 102.3 & 125.9 & 102.3 & 1259 & 1259 & 125.9 & 125.9 & 125.9 \\
\hline Deposits & $3,082.3$ & 766.2 & 649.4 & 642.4 & 741.4 & 606.2 & 839.0 & 568.8 & 901.4 & 952.4 & 970.9 & 982.0 & 989.4 \\
\hline \multicolumn{14}{|l|}{ Domeatic money banks (DMBr) } \\
\hline Net foreign assets & 886.1 & $1,015.8$ & 955.4 & $1,108.9$ & $1,035.9$ & $1,171.4$ & 9826 & 963.9 & $1,046.8$ & $1,053.1$ & $1,053.1$ & $1,093.1$ & $1,053.1$ \\
\hline Net damestic assets & $4,660.8$ & $4,822.6$ & $4,690.3$ & $4,901.9$ & 4,457.8 & $4,988.7$ & 4.655 .7 & $5,333.3$ & $5,128.8$ & $5,554.8$ & $5,709.2$ & $5,801.8$ & $5,863.6$ \\
\hline Bank reserves & $1,204.9$ & 888.8 & 751.8 & 744,8 & 878.9 & 700.5 & 964.8 & 671.2 & $1,027.3$ & $1,078.3$ & $1,096,8$ & $1,107.9$ & $1,115,3$ \\
\hline Liabilities to BCM & -27.2 & -27.2 & -26.5 & -26.5 & -22.1 & -26.5 & -21.3 & -26.5 & -21.3 & .21 .3 & -21.3 & -21.3 & -21.3 \\
\hline Credit to govenument (net) & $1,121.6$ & $1,583.4$ & $1,479.1$ & $1,579.6$ & $1,410.0$ & 1.615 .7 & $1,344.2$ & $1,929.6$ & $1,457.7$ & $1,477.9$ & $1,532.9$ & $1,390.9$ & $1,406.6$ \\
\hline Purchese of liquidity papes If & 0.0 & -0.4 & 0.0 & 369.8 & 0.0 & 413.5 & 0.0 & 7328 & 0.0 & 0.0 & 0.0 & 0.0 & 0.0 \\
\hline Net credit to government (budget) & $1,121,6$ & $1,583.8$ & $1,479.1$ & $1,209.8$ & $1,410.0$ & $1,202.2$ & $1,344.2$ & $1,196.9$ & $1,457.7$ & $1,477.9$ & $1,532.9$ & $1,390,9$ & $1,406.6$ \\
\hline Other clairs on public sector & 405.4 & 461.3 & 415.2 & 461.3 & $3 \mathrm{~B} 2.0$ & 461.3 & 368.8 & 461.3 & 368.8 & 368.8 & 368.8 & 368.8 & 368.8 \\
\hline Claims an public enterprises & 41.6 & 36.9 & 38.3 & 41.6 & 28.5 & 41.6 & 27.0 & 41.6 & 27.0 & 27.0 & 27.0 & 27.0 & 27.0 \\
\hline Credit to privale sector & $2,434.5$ & $2,605.1$ & $2,620.5$ & $2,706.6$ & $2,463.3$ & $2,793.8$ & $2,764.5$ & $2,851.9$ & $2,880.5$ & $3,084,0$ & $7,214.9$ & $3,388.4$ & $3,477.1$ \\
\hline Other items (net; assels +) & -520.0 & .725 .7 & $-589,0$ & -605.6 & -683.0 & -605.7 & -792.3 & -605.9 & -611.1 & 459.8 & -509.8 & $-459, \mathrm{~B}$ & -509.8 \\
\hline Deposits & $5,369,6$ & $5,662.2$ & $5,462.2$ & $5,827.2$ & $5,326.3$ & $5,976.6$ & $5,468.7$ & $6,113.7$ & $6,005.9$ & $6,438.2$ & $6,592.6$ & $6,585.2$ & $6,747,0$ \\
\hline Deposits in local currency $3 /$ & $4,490.7$ & $4,605.4$ & $4,434,2$ & $4,799.2$ & $4,330.4$ & $4,948.6$ & $4,454 . J$ & $5,085.7$ & $4,975.9$ & $\$, 402.0$ & $5,556,4$ & $5,649,0$ & 5.7108 \\
\hline Deposits in foreign currency (time deposita) & B7.9.9.9. & $1,059.1$ & $1,028.0$ & $1,028.0$ & 995.9 & $1,028.0$ & $1,014.6$ & $1,028.0$ & 1.030 .0 & $1,036.2$ & $1,036.2$ & $1,036.2$ & $1,036.2$ \\
\hline (in mitlians of SDRs) & 100.5 & 116.3 & 116.2 & 111.2 & 116.4 & 109,7 & 116.4 & 108.1 & 116.4 & 116.4 & 116.4 & 116,4 & 116.4 \\
\hline Short-tem bonds (liabilitles) & 177.4 & 176.2 & 183.5 & 183.5 & 167.3 & 183.5 & 169.7 & 1835 & 169.7 & 169.7 & 169.7 & 169.7 & 169.7 \\
\hline
\end{tabular}

Sourcest Central Bank of Madagascar (BCM); and Fund staff estimates and projections.

1/ Sales of treasury bills for monetary poltcy purposes.

2/ 2003 inchudes the takeover of slate oil company (SOLMA) debt (FMG 350.4 billica) by the government.

3/ Includes Compfes de Cheques Pastaux and Caisse d'Epangne de Jadagarcar deposit accounts.

CInternational Monetary Fund. Not for Redistribution 
Table 3. Madagaxer: Balance of Poymente, $2000 \mathrm{~m} 08$

(In mitjions of SDRs, unlcas otherwise indicalcd)

\begin{tabular}{|c|c|c|c|c|c|c|c|c|c|c|c|}
\hline \multirow{2}{*}{ 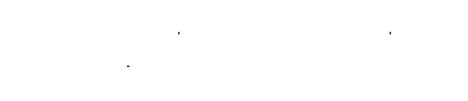 } & \multirow[t]{2}{*}{2000} & \multirow[t]{2}{*}{2001} & \multicolumn{2}{|c|}{20,12} & \multicolumn{2}{|c|}{2003} & \multirow{2}{*}{$\begin{array}{l}2004 \\
\text { Rev. } \\
\text { Prog. }\end{array}$} & \multirow[t]{2}{*}{2005} & \multirow{2}{*}{$\frac{2006}{\text { Projections }}$} & \multirow{2}{*}{2007} & \multirow[t]{2}{*}{ 200k: } \\
\hline & & & Prog & Prel. & Prag. & Proj. & & & & & \\
\hline Cument gecautet & -1654 & $\$ 6.4$ & -1550 & .2035 & -168.6 & -160.4 & -231.3 & .1412 & -262.6 & -276.3 & -297.7 \\
\hline Goods and arvicos & -214.8 & -114.3 & 202.4 & -224.8 & -275.9 & -300.4 & -360.4 & -371.2 & -394.1 & -409.8 & -490.6 \\
\hline J tapte tance & -78.6 & 11.6 & -91.3 & -90.5 & -90.1 & -154.5 & -225.1 & -216.9 & -214.9 & -185.3 & -172.7 \\
\hline Exports & 628.5 & 757.9 & 405.0 & 375.0 & 584.9 & 566.0 & 613.5 & 757.6 & 881.1 & $1,01+.6$ & $1,115.5$ \\
\hline Imports & -707.1 & -745.3 & -497.3 & +55.5 & -675.8 & -720.9 & -392.6 & -974.5 & -1.0950 & $-1,199.8$ & $-1,288.2$ \\
\hline Net erviess (not) & -136.2 & -125.9 & -111.1 & .134 .3 & -135.8 & -134.5 & .135 .2 & $-156,4$ & -179.2 & -24.5 & -257.9 \\
\hline Services, receiquts & 370.9 & 276.3 & 181.1 & 179.1 & 247.3 & 235.7 & 2B7.B & 315.4 & 346.0 & 361.8 & 377.8 \\
\hline Strvitan paymenta & -407.1 & -1022 & $-292,3$ & .307 .4 & -433.1 & -390.2 & -29.1 & -469.8 & -535.2 & -586.5 & -535.7 \\
\hline In oume (Bat) & $-53,3$ & -46.7 & -38.3 & .59 .0 & .96 .4 & -579 & -47.9 & -38.0 & -43.9 & -51.8 & -63.0 \\
\hline Receips & 16.6 & 18.7 & 15.5 & 20.1 & 193 & 7.9 & 15.7 & 200 & 21.2 & 21.5 & 23,6 \\
\hline Payments & -69.9 & -65.4 & -74.0 & -73.1 & .75 .7 & -65.8 & -52.6 & -58.0 & -65.1 & -74 & +86.6 \\
\hline Of whth: gaverumant ineerest ${ }^{1}$ & -39.6 & $-4 ! 4$ & -43.9 & -49.1 & -42.9 & -40.1 & -42.4 & -29.9 & -29.0 & -27.9 & $-27,0$ \\
\hline Current tranafens & 102.6 & 1146 & $\cos .9$ & 74,2 & 169.6 & 179.9 & 177.9 & 1680 & 175.4 & 185.3 & 195.9 \\
\hline Govermmost & 25.5 & 24.9 & 44,9 & 6.2 & 98.3 & 983 & 92.9 & 75.9 & 79.9 & 84.1 & 88.6 \\
\hline Budget aid & 16.5 & 36.7 & 51.3 & 21.1 & $\mathbf{8 8 . 8}$ & $\$ 1.9$ & B2.5 & 66.4 & 70.4 & 74.6 & 79.6 \\
\hline HIPC robict ${ }^{2}$ & 0.0 & 18.8 & 20.9 & 20.2 & 2.2 .2 & 22.2 & 19.9 & 0.0 & 0.0 & 0.0 & 0.0 \\
\hline Grante & 16.5 & 17.8 & 31.1 & 1.9 & 66.7 & 66.7 & 62.6 & 66.4 & 70.4 & 74.6 & 79.1 \\
\hline Other (wet) ${ }^{3}$ & 9.0 & -11.8 & -6.4 & -15.5 & 9.5 & 10.4 & 10.4 & 9.5 & 9.5 & 9.5 & 9.5 \\
\hline Private & 77.1 & 89.7 & 61.0 & 68.0 & 65.3 & 74.6 & 85.0 & $9+1.1$ & 95.9 & 겨여논 & 1073 \\
\hline Capital and fumeinal boocunt & $7 \downarrow .6$ & 65.9 & 67.5 & 104.1 & 168.2 & 166.9 & 285.2 & 3053 & 314.9 & 3278 & 344.6 \\
\hline 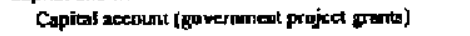 & 17.0 & BB.6 & 40.4 & 44.7 & 65.9 & $6: 8$ & 124.5 & 132.0 & 199.9 & 148.3 & 1572 \\
\hline Pinancial account & 34.0 & 90.9 & 27.1 & 25.5 & $104 I$ & 33.9 & 163.7 & 173.5 & 175.0 & 175 & 187.4 \\
\hline Direct investrinent & 52.9 & 79.1 & 5.0 & 64 & 13.1 & 9.1 & 52.0 & 60.6 & 60.6 & 69.6 & 60.6 \\
\hline Of which privatization reccipes & 5.3 & 10.9 & 3.0 & 33 & 3.7 & 0.0 & 0.0 & 0.0 & 0.t & 00 & 0.0 \\
\hline Other & $-\$ 8.9$ & 17.8 & $\cdot 22.1$ & 19.1 & 91.1 & 24.2 & 111.7 & 112.9 & 114.4 & 118.9 & 126.8 \\
\hline Govemingent & 6.1 & 21.5 & 54.6 & 48.1 & 96.6 & 49.18 & 117.0 & 1173 & 119.4 & 126.5 & 134.3 \\
\hline Drawing & 86.9 & 90.2 & 126.7 & 120.3 & 167.6 & 120.8 & 186.1 & 164.2 & 172.9 & 183.3 & 194.5 \\
\hline Prajent drawiogs & 46.2 & 60.4 & 47.4 & 54.6 & 105.0 & 83.5 & 107.5 & 136.2 & 141.3 & 151.7 & 162.9 \\
\hline Budgetsary nuppoet & 39.3 & 26.8 & 79.3 & 65.7 & 36.6 & 37.3 & 78.6 & 28.0 & 31.6 & 31.6 & 31.6 \\
\hline Non-bovermirent & 1.4 & 3.0 & a.t. & b.0 & 6.0 & $\mathbf{0 . 0}$ & 0,0 & 0.0 & 0.0 & 0.0 & a. \\
\hline Amertiection' & 280.8 & .58 .7 & .72 .1 & -72.1 & $-3 ! \cdot 0$ & -31.0 & -69.1 & $-\mathbf{4 . 3}$ & -39.5 & -36.2 & -80.2 \\
\hline Private sector anortization & 0.0 & -7.5 & -7.5 & -7.9 & -5.4 & -7.7 & -5.3 & -4.4 & -3.0 & .7 .6 & -7.4 \\
\hline Benkes, not & -25.0 & 31 & .25 .0 & -21.1 & $\mathbf{d . 0}$ & -17.1 & 0.0 & 0.0 & 0.0 & 0.6 & 0.0 \\
\hline Other (ind, crrors and onissions) & -49.4 & $-113,6$ & 0.0 & 39.9 & 0.0 & 69.2 & 0.0 & 0.0 & 0.0 & 0.0 & 0.0 \\
\hline Oveas if tolance & .93 .8 & $19: 6$ & -87.5 & -99.4 & $-0,4$ & -26.5 & 5.9 & 62.3 & 32.3 & 51.5 & 46.9 \\
\hline Finaweinf & 93.8 & .19 .6 & 87.5 & 99.4 & 0.4 & 26.5 & -57.9 & -62.3 & $-\$ 2.3$ & -51.5 & -16.9 \\
\hline Net fodtign anet (increase -) & $2 \mathrm{~B}, 4$ & -76.9 & .30 .4 & 40.0 & -401 & -140 & -96.7 & .117 .5 & -109.7 & -108.8 & -105.1 \\
\hline Use of Fund wradit (nct) & 34.2 & 21.4 & 8.6 & 8.6 & 17.3 & 5.5 & 173 & 3.2 & -16.9 & -17.5 & $\cdot .7 .6$ \\
\hline Disburbexinarits & $3 \mathrm{BO}$ & 227 & เ..4 & $11 A$ & 22.7 & $\$ 1.3$ & 22.7 & 11.3 & 0.0 & 0.0 & 0.0 \\
\hline Repryments & .3 .8 & $-6,3$ & -2.7 & -2.7 & -5.4 & .5 .4 & -54 & $-\mathbf{R} 1$ & -16.9 & -17.6 & -.7 .6 \\
\hline Other buter net (incrane -) & -5.7 & -98.3 & 21.4 & 31.4 & $-57,4$ & -20.0 & $-114,0$ & -120.8 & -92.9 & .91 .2 & $-\$ 7.6$ \\
\hline Net change in artears (exciluding ceneral bank) ${ }^{s}$ & 5.8 & 1.6 & 0,0 & 1.3 & -9.5 & -9.5 & 0.0 & B.t & 0.0 & $0: 0$ & 0.0 \\
\hline Debt relicf and cancellation ${ }^{67}$ & 59.6 & $\$ 5,8$. & 575 & $\$ 7.9$ & 50.0 & 500 & 38.8 & 55.3 & 57.5 & 57.3 & 58.2 \\
\hline \multicolumn{12}{|l|}{ Mormonandem itctinet } \\
\hline Grante (in peroent of GDP) & 3.2 & 3.2 & 2.5 & 1.5 & 4.4 & 42 & 5.1 & 4,6 & 4.5 & 4.4 & 4.4 \\
\hline Losn (in pereent of (RDP) & 0.2 & 0.6 & I.6 & 1.4 & 2.6 & 13 & 2.8 & 2.6 & 2.4 & 2.4 & 24 \\
\hline Direct imvestment (in pereent of GDP) & 1.8 & 2.1 & 0.1 & 0.2 & 0.4 & 0.2 & 1.2 & 1.3 & 1.2 & 1.2 & 1.1 \\
\hline \multicolumn{12}{|l|}{ Cuorent acopusk (îr partant of GDP) } \\
\hline Excluding nct official unensers & $-6,5$ & -2.16 & -3.8 & .6 .1 & -7.3 & -7.5 & -7.6 & -7.0 & -7.0 & -6.9 & -6.9 \\
\hline Inthuding net oftieial unandens & -5.6 & -1.3 & $-4,5$ & -5.5 & -4.6 & -49 & -9.4 & -5.4 & -5.4 & -3.3 & -3.3 \\
\hline Grocs offioial reserves & 218.7 & 317.5 & 296.1 & 266.6 & 3240 & 286.6 & 400.6 & 521.3 & 6142 & 705.4 & 795.0 \\
\hline (in menths of tinporits of gurods and nonfartor $s$ & $3: 4$ & 3.3 & 49 & 4.1 & 3.5 & 3.1 & 3.8 & 4.3 & 4.5 & 4.7 & 4.9 \\
\hline \multicolumn{12}{|l|}{ Exchange rates } \\
\hline Malogesy frates/SDR (pariod avarage) & $8,951.0$ & 8,351 & 8.895 .0 & $8,774,3$ & $\ldots$ & $\ldots$ & $\ldots$ & $\ldots$ & $m$ & $\cdots$ & $\cdots$ \\
\hline Malagary tancesU.S. dolley (period average) & $6,787.2$ & $6,591.5$ & $6,8 \mathrm{BO} . \mathrm{l}$ & $6,592.3$ & $\cdots$ & $\cdots$ & $\cdots$ & $\cdots$ & $\cdots$ & $\cdots$ & $\cdots$ \\
\hline
\end{tabular}

Söpces: Central Bank of Madagecor, Ministry of Finance, DMP Pinance Dept, and Fuad staff edimithes and projestions.

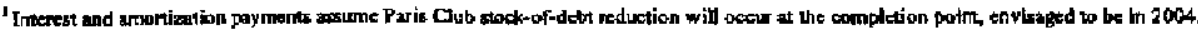

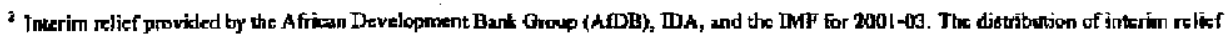

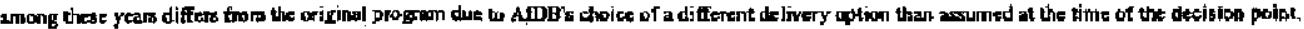

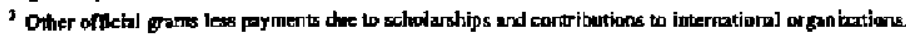

4 Intuds commereial enedits racoived or grented

'From $2000 \mathrm{~m}$, includes external cummenial arrears.

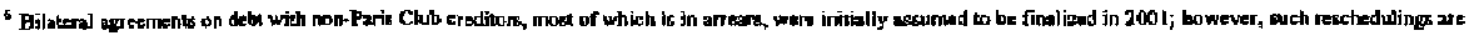

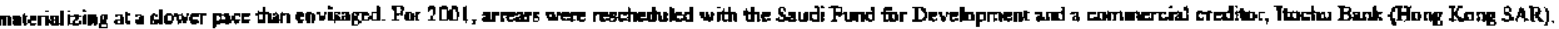

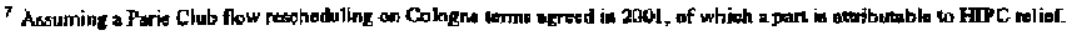

- In percent of exports of goods and nonfactor tervices.

'In persent of govemment revino 
Tabte 9. Madegaxear: External Debt Sustainsbifity Indícators, 2000-19'

\begin{tabular}{|c|c|c|c|c|c|c|c|c|c|c|c|}
\hline & 2000 & 2001 & 2002 & 2003 & 2004 & 2005 & 2006 & 2007 & 2008 & $\begin{array}{l}\text { Average } \\
2009 \mathrm{a} \cdot \mathrm{ds}\end{array}$ & $\begin{array}{l}\text { Arengo } \\
2010-19\end{array}$ \\
\hline $\begin{array}{l}\text { Afere traditionat debt relier } \\
\text { Cuncul esthmates }\end{array}$ & & \multirow{2}{*}{\multicolumn{10}{|c|}{ (To pencent) }} \\
\hline 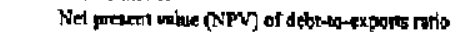 & 220,4 & 192.7 & 2113 & 225.4 & 239.2 & 1936 & 178.2 & 150.4 & 148.1 & 182.8 & \\
\hline NPV at dqBS-ro-seythue tativ & 4700.7 & 476.3 & 624.7 & 421.5 & 352.0 & 330.0 & 307.4 & 296,1 & 267.2 & 382.7 & 182.3 \\
\hline NPV of debl-ta-ODP ntio & 55.0 & 48.4 & 50,0 & 49.5 & 42.0 & 40.5 & 390 & 37,6 & 36.0 & $4 \mathrm{k} .2$ & 270 \\
\hline Deba service-to-expots ratio & 12.9 & 10.6 & 11.9 & 120 & 12.8 & 10.1 & 9.6 & 8.6 & 8.0 & 10.0 & 4.2 \\
\hline 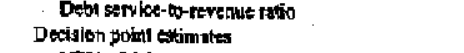 & 27.6 & 26.2 & 35.2 & 22.4 & 18.8 & 16.8 & 16.6 & 15.3 & 14.5 & 20.8 & 8.1 \\
\hline NPV afdebt-10-expers ratio & 239.7 & 229.0 & 220.4 & 210.0 & 19.4 .4 & 180.7 & 168.5 & เ\$9.9 & 151.8 & 192.0 & 111.0 \\
\hline NPV of debt-to-reverue natio & 439.6 & 809,4 & 375.4 & 343.1 & 313.3 & 286.3 & 262.1 & 240.3 & 220.7 & 289.0 & 127.8 \\
\hline NPV of debt-to-ODP rodo & 55.0 & 522 & 48.5 & 453 & 423 & 39.5 & 37.0 & 34.6 & 32.4 & 39.6 & 21.6 \\
\hline 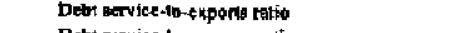 & 11.7 & 11.2 & 10.2 & 9.8 & 9,3 & 9.9 & 9.5 & 9.2 & 9.6 & 9.8 & 7.3 \\
\hline Debt anvies-to-revanue ratio & 23.1 & 21.4 & t8.8 & 17.4 & 16.7 & 15.5 & 15.9 & 14.9 & 14.0 & 17.1 & 9.4 \\
\hline \multicolumn{12}{|l|}{ A Alere enhanced HPC wositance } \\
\hline \multicolumn{12}{|l|}{ Currut estimaten 2} \\
\hline NPV ige dtat-to-expoth natio & 259.2 & $i 82.6$ & 129.7 & 142.2 & 354,6 & 131.1 & 120.2 & 110.5 & 104,0 & 134.6 & 85.7 \\
\hline & 553.5 & 451.8 & 363.3 & 265.9 & 212.5 & 217.8 & 207.4 & 197.8 & 187.5 & 281.8 & 147.6 \\
\hline NPV of dobe-6a-GDP ratio & 64.7 & 45.9 & 30.7 & 27.5 & 27.1 & 36.7 & 26.3 & 25,9 & 25,3 & 30,3 & 27,4 \\
\hline Debt service-to-enports twio & 6.7 & 3.9 & 4.7 & 5.1 & 70 & 5.6 & 5.3 & 4.7 & 4,4 & 5.0 & 2.0 \\
\hline 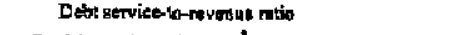 & 14.4 & 9.8 & 13.8 & 9.3 & 20.3 & 9.4 & 9.1 & 8.4 & 7.9 & 10.5 & 3.4 \\
\hline \multicolumn{12}{|l|}{ 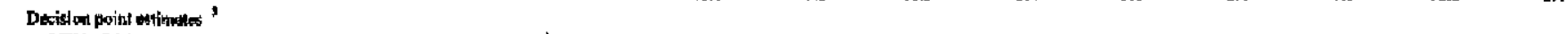 } \\
\hline NPV ne detht-to-exports ratio & 281.6 & 2172 & 276.7 & 133.1 & 129.1 & 118.3 & 112.0 & 108.1 & 104.4 & 126.4 & $\$ 9.3$ \\
\hline NPV of doth-la-reveave reto & $\$ 11.6$ & 388.4 & 232.8 & 217.5 & 201.6 & 187.3 & 174.3 & 162.5 & 151.8 & 206.5 & 106.1 \\
\hline NPY of deter-ta-GDP ratio & 0.5 & 49.5 & 30.1 & 28.7 & 27.2 & 25.9 & 24.6 & 23.4 & 22.3 & 28.1 & 17.6 \\
\hline Debit service-la-exports ntio & 11.7 & 5.2 & 5.4 & 3.5 & 5.6 & 5.3 & 60 & 6.0 & 6.3 & 5.7 & 4.5 \\
\hline Debt service-to-rwentue patio & 21.2 & 19.8 & 9.2 & 9.7 & 10.0 & 9.3 & 10.1 & 9.7 & 9.2 & 9.8 & 6.3 \\
\hline Mentorandum iterrs: & \multirow{2}{*}{\multicolumn{11}{|c|}{ (In millians of US. dalkas) }} \\
\hline GDP & & & & & & & & & & & \\
\hline Current estinater & 3,868 & 4,527 & 4,557 & $\$, 459$ & 5,093 & 6,327 & 6,793 & 7,293 & 7.830 & 6,697 & 12,866 \\
\hline Decision print estimaleo & 3,906 & 4,283 & 4,793 & 5,291 & 5,006 & 6.376 & 6,960 & 7,598 & 8,295 & 6.234 & 15.138 \\
\hline \multicolumn{12}{|l|}{ Exports (1trete-yed moving avcragc) } \\
\hline Ourrent escimatess & 965 & 1,137 & 1,097 & 1,054 & 1,034 & 1,290 & 1,487 & 1,708 & 1,901 & 1,374 & 3,366 \\
\hline \multirow{2}{*}{\multicolumn{12}{|c|}{$\begin{array}{l}\text { Decision print estimituts } \\
\text { Experts (eurntent year) }\end{array}$}} \\
\hline & & & & & & & & & & & \\
\hline Current entintest & 1,186 & 1,316 & 730 & 1,117 & 1,256 & 1,496 & 1,710 & 1,918 & 2,081 & $1,50 \%$ & 3,657 \\
\hline Deflaion point estrimater & 972 & 1,046 & 1,195 & 1.242 & 1,412 & 1,328 & $\mathrm{~L}, 54 \mathrm{l}$ & 1,767 & 1,510 & 1,472 & 3.160 \\
\hline \multicolumn{12}{|l|}{ 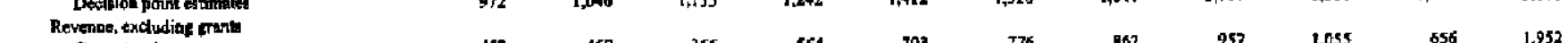 } \\
\hline Current notimales & 452 & 460 & 365 & 564 & 703 & 776 & 862 & 957 & 1,055 & $\begin{array}{l}856 \\
867\end{array}$ & 1,952 \\
\hline \multirow{2}{*}{\multicolumn{10}{|c|}{ 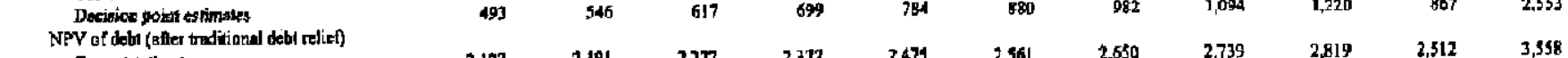 }} & & \\
\hline & & & & & & 2,561 & 2,550 & 2.739 & 2,819 & 2,512 & 3,598 \\
\hline $\begin{array}{l}\text { Current estizattes } \\
\text { Decision paimt rtimates }\end{array}$ & $\begin{array}{l}2,127 \\
2,147\end{array}$ & 2,235 & 2,316 & 2,397 & 2,456 & 2.519 & 2,573 & 2,630 & 1,691 & 2,072 & 3,264 \\
\hline \multicolumn{12}{|l|}{ 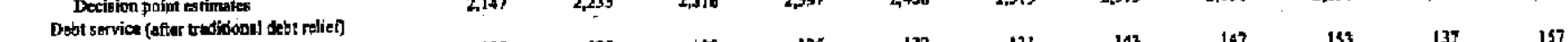 } \\
\hline Current estimates & 125 & 120 & 128 & 126 & 132 & 131 & 143 & 143 & 133 & 137 & 157 \\
\hline Decision point antimates & 114 & 117 & 116 & IM & 131 & 137 & 156 & 163 & 171 & 14! & 229 \\
\hline \multicolumn{8}{|l|}{ 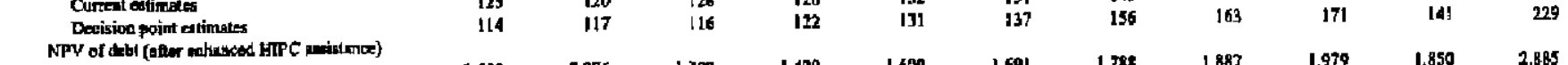 } & & & 1,850 & $2 \mathrm{gBS}$ \\
\hline Cunnent estimnders & 2,502 & 2,076 & $1, \sqrt{97}$ & 1,499 & 1,690 & 1,691 & $\begin{array}{l}1,788 \\
1,711\end{array}$ & $\begin{array}{l}1,887 \\
1,778\end{array}$ & $\begin{array}{l}1,979 \\
1,851\end{array}$ & 1,850 & $\begin{array}{l}2,8,85 \\
1,991\end{array}$ \\
\hline 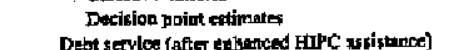 & 2.521 & 2,120 & 1,436 & 1,519 & 1,581 & 1,649 & 1.711 & & & & \\
\hline 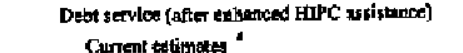 & 53 & & 50 & 54. & נד & 73 & 79 & 81 & 83 & 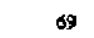 & 67 \\
\hline $\begin{array}{l}\text { Cunent tetimater “" } \\
\text { Decosion point atimstes }\end{array}$ & $\cos$ & 4 & 36 & 68 & 79 & 2 & 99 & 106 & 112 & 89 & 156 \\
\hline
\end{tabular}

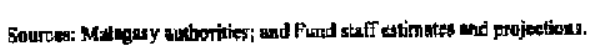

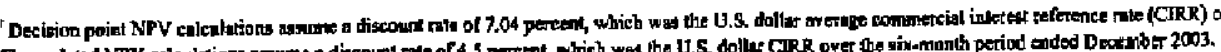

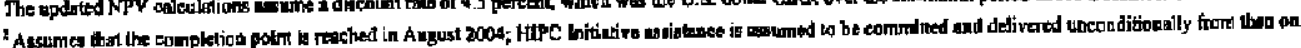

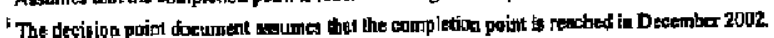

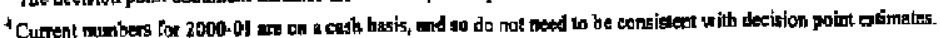

CInternational Monetary Fund. Not for Redistribution 
Table 10, Madaganear: Sourtes and Unos of Kesourses, 2001.08

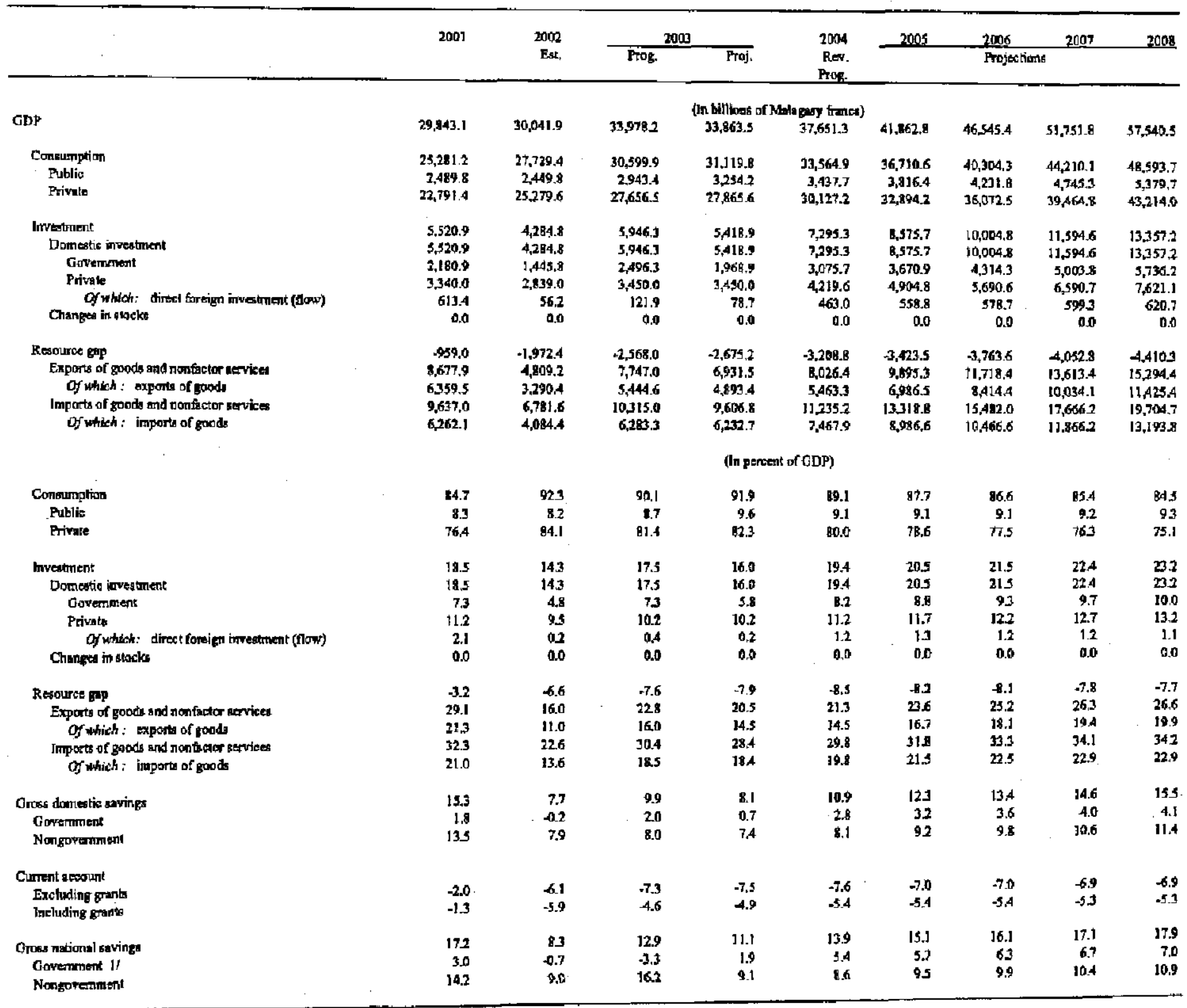

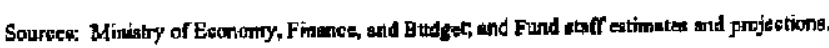

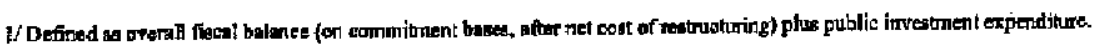


Table 11. Madagascar: Trecking Delivery of HIPC Assistance

\begin{tabular}{|c|c|c|c|c|c|c|c|c|c|}
\hline & \multirow{2}{*}{$\begin{array}{r}\text { Conbact with } \\
\text { Credizur } \\
\text { Date. }\end{array}$} & \multicolumn{2}{|c|}{$\begin{array}{l}\text { Agreement to } \\
\text { Provide GitipC A Asictance }\end{array}$} & \multicolumn{2}{|c|}{ 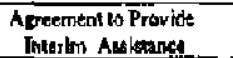 } & \multicolumn{2}{|c|}{$\begin{array}{l}\text { Delivery of Interins } \\
\text { Assistame: }\end{array}$} & \multirow{2}{*}{$\begin{array}{r}\text { Delivery of } \\
\text { Completion. Pt. Assias. } \\
\text { Date }\end{array}$} & \multirow[b]{2}{*}{ Comments } \\
\hline & & Status & Date & $\operatorname{sen}$ & Dare & Statere & Dale & & \\
\hline \multicolumn{10}{|l|}{ Multilatural ereditors } \\
\hline TMP & 18.Def-000 & $y=0$ & |E-Apr-01 & $y=$ & & $y=$ & & & \\
\hline Tox & $21-D e c-0\}$ & yes & 2T.Deg-100 & yes & & yes & & & \\
\hline 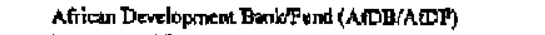 & $|9-F \in b=0|$ & $y=$ & $2 t-\mathrm{Apr}-01$ & yes & & you & & & \\
\hline 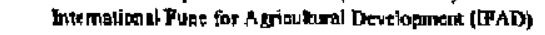 & 19-Feb-O1 & yes & $18-0 \mathrm{ct}-01$ & m & & no & & & Ansistance will be provided at completion paint. \\
\hline Eumopean Union/European Investritend Bank (EU/EIB) & 19.Feb-01 & yet & $16-527-03$ & yes & & no & & & Modaltites to be weed \\
\hline OFEC & 19. Fcb-01 & yea & 76-Feb-0? & finnined & & yat & & & Agrerment \\
\hline Anab Bank for Ecomencia Development in Africa (BADE & |9-Feb-01 & you & I5-Apr-01 & no & & no & & & Agsitstance with be pervided an completion point. \\
\hline \multicolumn{10}{|l|}{ Paris Club credithors ${ }^{1}$} \\
\hline Austria & & yes & $10-00 \pi-01$ & yes & & yes & & & Agresment on Calog ma terms. \\
\hline Belgium & & $y \times$ & 7.Mar-01 & yes & & yes & & & 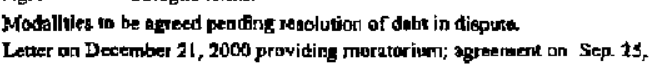 \\
\hline Canads & 21-Ded-100 & yes & 7.,Mar-01 & yets & S-Nov-02 & yef & & & 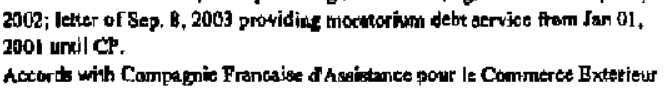 \\
\hline Franse & & yete & 7-Mar-01 & yes & & yea & & & 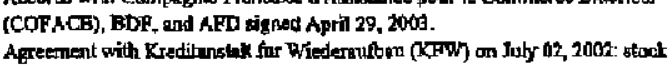 \\
\hline Germany & & $y \neq$ & 2-5ul-02 & yes & 2-Kulater & yet & & & and flow. Agreement whth Bermen on January 31,2003 . \\
\hline Isread & & yes & 7-Мat-01 & yos & & yes & & & Agrwment nigned Oraber 3, 2003. \\
\hline Ttaly & & yed & 22-May 01 & yes & 22-May-01 & $y=8$ & & & Letter * 1712 anzouncing decisiad to tarkel I00 percent of debt. \\
\hline Inpan & & yes & 7-Mer-0! & jen & & 幽 & & & \\
\hline Ruseria & & yes & 7-Mar.0I & yes & & yes & 6-Deco.01 & & 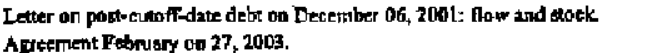 \\
\hline Spadn & & yes & 20-Sep-01 & $y=8$ & & yes & Gedecat & & $\begin{array}{l}\text { Aptefment Fotbrusry on 27, } 2003 . \\
\text { Agreernetit on Cologne terms. }\end{array}$ \\
\hline Switze:land & & yes & [E-0ert:01 & yet & 22-May-0! & yes & $|\mathrm{B}-\mathrm{OHe}-\mathrm{a}|$ & & 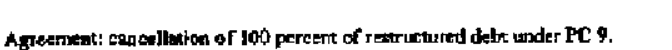 \\
\hline Sweder & & yes & 7-Mar-0l & yes & & $y=$ & & & \\
\hline Unloed Kingdom & & $y=a$ & 7-Mar-01 & yes & & yen & & & 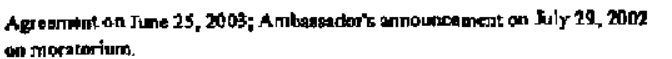 \\
\hline United Srater. & & $y=a$ & 7-Marnol & yos & & yes & & & \\
\hline \multicolumn{10}{|l|}{$\begin{array}{l}\text { Dhited Srater } \\
\text { Nom-Par is Club bikterat crediuns }\end{array}$} \\
\hline Algerie & 5-.Now-01 & mo & & na & & $\mathbf{m}$ & & & \\
\hline Angola & B-Now-01 & tho & & no & & mo & & & A reement refief of 25.94 perceent of the tatal stock of dett the govenument is \\
\hline Chirre & $2-$ Now & yes & & yes & & yet & [1-Now-BI & & 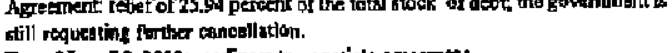 \\
\hline Kuwait & S-Nov-01 & no & & nó & & no & $27-5 e p-99$ & & Negotiations under way. \\
\hline Libyt & 3-Nov-01 & no & & no & & ת & & & \\
\hline United Arab Emlratel & 3-Nov-01 & no & & no & & no & & & \\
\hline Seudi Arabin & S-Nov-01 & חo & & no & & no & 24-Jarncl & & \\
\hline \multicolumn{10}{|l|}{ Commercial credicans } \\
\hline \multirow{2}{*}{\multicolumn{10}{|c|}{ Orwhtat }} \\
\hline $\begin{array}{l}\text { AGP } \\
\text { GNT Intematimal Lx Eank }\end{array}$ & & & & & & & & & \\
\hline Harg Kong HITOCHU & & & & yes & $27-A p r-01$ & yes & & & Agreemem \\
\hline C-andon C & & & & & & & & & \\
\hline
\end{tabular}

Sources: Malagasy authorlies; and IMUt soff.

'Paris Club atreed minutes on restructuring from Decernber 2000 to HHPC floxing eompletion point. 
Table 12. Madagascar: Regional Poverty by Area of Residence, 1993-2002 (In percent)

\begin{tabular}{|c|c|c|c|c|c|c|c|c|c|c|}
\hline & \multicolumn{4}{|c|}{ Head Count ' } & \multirow[b]{2}{*}{2002} & \multicolumn{5}{|c|}{ Depth of Poverty } \\
\hline & 1993 & 1997 & 1999 & 2001 & & 1993 & 1997 & 1999 & 2001 & 2002 \\
\hline \multicolumn{11}{|l|}{ Urban poverty } \\
\hline Total & 50.1 & 63.2 & 52.1 & 44.1 & 61.6 & 17.5 & 29.6 & 21.4 & 18.3 & 29.3 \\
\hline Antananarivo & 42.4 & 52.0 & 43.3 & 28.3 & 51.2 & 15.9 & 23.0 & 17.5 & 10.4 & 23.1 \\
\hline Fianarantsoa & 64.9 & 83.1 & 55.8 & 59.8 & 78.5 & 22.4 & 42.0 & 25.2 & 25.5 & 42.4 \\
\hline Toamasina & 55.8 & 76.3 & 52.6 & 60.1 & 66.9 & 18.5 & 39.9 & 21.1 & 28.4 & 33.2 \\
\hline Mahajanga & 37.3 & 68.2 & 65.2 & 49.8 & 71.0 & 11.6 & 23.2 & 25,3 & 17.3 & 33.6 \\
\hline Toliara & 66.9 & 69.1 & 66.5 & 53.3 & 58.3 & 25.0 & 37.3 & 29.8 & 25.4 & 25.1 \\
\hline Antsiranana & 49.5 & 27.0 & 31.3 & 30.1 & 62.5 & 14.3 & 6.2 & 7.8 & 8.7 & 28.1 \\
\hline \multicolumn{11}{|l|}{ Rural poverty } \\
\hline Total & 74.5 & 76.0 & 76.7 & 77.1 & 86.4 & 33.1 & 34.7 & 36.1 & 39.7 & 53.0 \\
\hline Antananarivo & 76.2 & 72.1 & 69.3 & 56.7 & 72.3 & 31.6 & 31.5 & 29.5 & 25.5 & 38.1 \\
\hline Fianarantsoa & 75.3 & 73.6 & 85.9 & 87.9 & 93.5 & 35.3 & 30.1 & 43.1 & 49.4 & 60.7 \\
\hline Toamasina & 81.1 & 80.8 & 76.4 & 87.9 & 91.9 & 36.0 & 39.2 & 35.7 & 48.2 & 57.1 \\
\hline Mahajanga & 56.7 & 75.1 & 78.8 & 78.4 & 93.8 & 20.2 & 30.6 & 39.4 & 39.9 & 63.8 \\
\hline Toliara & 84.2 & 84.9 & 73.1 & 83.3 & 87.5 & 46.5 & 48.5 & 34.8 & 43.5 & 53.5 \\
\hline Antsiranana & 63.7 & 69.5 & 80.6 & 79.0 & 88.6 & 24.5 & 27.5 & 36.7 & 34.0 & 56.7 \\
\hline National poverty & 70.0 & 73.3 & 71.3 & 69.6 & 80.7 & 30.3 & 33.6 & 32.9 & 34.8 & 47.6 \\
\hline
\end{tabular}

Source: INSTAT, Cornell University; and World Bank staff estimates from household survey data.

1/ Head count measures the share of population with per capita consumption below the poverty line. Based on household surveys conducted in 1993, 1997, 1999, 2001, and 2002. 
Table 13. Madagascar: Basic Social and Demographic Indicators ${ }^{1}$

Land area (square kilometers)

Population

Total (in millions) (2001)

16.0

Urban population (percent of total)

30.1

Population density (people per sq. km.)

26.7

Population density, rural (people per sq, km, of arable land) (1998)

407.6

Population growth (annual percentage change)

GNI per capita (in U.S. dollars, World Bank Atjas method, 2001)

Life expectancy at birth (iw years) (2001)

Overall

Womer.

Men

Crude birth rate (per 1,000) (1999)

40.7

Cude death rate (per 1,000) (1999)

12.1

Fertility rate (percent) (1999)

Infant mortality rate (per 1,000) (2001)

Education

Illiteracy rate, adult total (percentage of people over 15) (2001)

Primary education, pupils (in thousands) (1998)

Secondary education, general pupils (in thousands) (1997)

356.6

Secondary education, vocational pupils (in thousands) (1992)

8.1

Primary school gross enrollment rate (percentage of relevant age group) (2001)

68.0

Secondary school gross enrollment rate (percentage of relevant age group) (1999) ${ }^{2}$

19.4

Tertiary school gross enrollment rate (percentage of relevant age group) (1995)

Health

Hospital beds (per 1,000) (1999)

Physicians (per 1,000) (1999) ${ }^{2}$

Safe water (percentage of population with access) (2001) $\quad 47.0$

Rural

Urban

Sanitation (percentage of population with access) (1999)

Rural

Urban

Child immunization (under 12 months; percent) (1999)

DPT

$\begin{array}{lr}\text { HIV prevalence (percent) (1999) } & 0.70\end{array}$

Sources: World Bank, World Development Indicators2001 (available on CD-ROM); and Malagasy authorities.

1 Most recent estimates available, unless otherwise indicated.

2 Source is the authorities' PRSP, IMF Country Report No. 03/323, (10/17/03). 
Table 14. Structural Pefformance Criteria, Structural Benchmarks, and Prior Actions for the Fourth Review of the PRGF-Supported Program

\begin{tabular}{|c|c|c|}
\hline & Timing & Status \\
\hline \multicolumn{3}{|l|}{ Structural performance criteria } \\
\hline $\begin{array}{l}\text { New regulations will be adopted, in consultation with the Fiscal Affairs Department of the Fund, } \\
\text { introducing a system authorizing the deferment of the value-added tax (VAT) payment obligations on } \\
\text { capital goods imports until the monthly declaration following the import. }\end{array}$ & By June 2003 & Done \\
\hline Draft budget execution laws for 2000 and 2001 will be submitted to the Audit Court & By end-December 2003 & Done \\
\hline \multicolumn{3}{|l|}{ Structural benchmarks } \\
\hline $\begin{array}{l}\text { An activity plan will be drawn op for the central bank's intemal audit department, along with an } \\
\text { organizational chart for intemal audit supervision by the various components of the bank. }\end{array}$ & By end-March 2003 & Done \\
\hline Treasury's monthly balance sheets up to end-2001 will be prepared. & By end-June 2003 & Not met \\
\hline An internal audit of the management of central bank reserves will be conducted. & By end-June 2003 & Done \\
\hline $\begin{array}{l}\text { The general shareholders' meeting of the oil company SOLIMA will examine the accounts for fiscal- } \\
\text { year } 2001 \text {. }\end{array}$ & By end-July 2003 & Done \\
\hline \multicolumn{3}{|l|}{ Prior actions } \\
\hline $\begin{array}{l}\text { Submission to Parliament of the } 2004 \text { budget law incorporating the tax policy measures presented in } \\
\text { MEFP Tabte } 3 \text {. }\end{array}$ & By end-December 2003 & Done \\
\hline $\begin{array}{l}\text { Collection of the full amount owed to the govenment by the oil companies in connection with the } \\
\text { liquidation of SOLIMA. }\end{array}$ & By end-December 2003 & Done \\
\hline Launching of international tender for the placement of JIRAMA under a maragement contract. & By end-January 2004 & Done \\
\hline
\end{tabular}




\section{Madagascar: Progress Toward HIPC Initiative Completion Point Triggers}

The triggers for the floating completion point under the enhanced HIPC Initiative are reported in Box 8, page 24 of the HIPC Decision Point Document for the Enhanced HIPC Initiative (12/01/00), (www,imf.org). The specific triggers (in addition to the implementation of a full PRSP for at least one year, the use of budgetary savings from interim debt-service relief in accordance with the criteria set at the decision point, and the maintenance of macroeconomic stability through satisfactory implementation of the PRGF-supportcd program) include the following: (A) actions in the area of financial monitoring and control; (B) the establishment of a public and transparent information system for the granting of licenses in the mining, fishing, and forestry sectors; and (C) the improved delivery of basic services.

\begin{tabular}{c|c|c}
\hline Triggers & Status & Comments \\
\hline
\end{tabular}

\section{A1, Improve financial monitoring and control as follows:}

Strengthen control systems through:

(i) adoption of an appropriate legal and constitutional framework;

(ii) adoption of procedures and internal control systems that conform to international technical standards; and

(iii) an increase in staffing and resources of the State Inspectorate General (IGE) and the Commitment Control Office (CDE).
Reforms cover the Audit Court (Chambre des Comptes) and the Procurement Commission (Commission des Marchés). The related plan of action centers on reforming the statutes governing these agencies, including assigning external auditing functions to the Procurement Commission and internal auditing functions to the State Inspectorate General.

Partially Revised legislative texts were formally submitted to completed parliament in December 2003. (NOTE: 2 of the revised legislative texts were submitted in Dec 2003, but 4 more will be submitted at the next session of Parliament).

Partially Detailed procedural manuals, increased reporting completed obligations of public institutions, and reinforcement of equipment and staffing of control agencies are fally completed. The systems will be effective after all the texts are approved by the parliament.

Completed Initial actions included the recruitment of additional staff for the agencics during 2001. Additional resources have been assigned to the agencies in the budget since 2002. Additional staff were recruited in 2003 for the IGE and CDE. Recruitments will be expanded in 2004. 


\begin{tabular}{|c|c|c|}
\hline Triggers & Status & Comments \\
\hline
\end{tabular}

Design a monitoring system for the budgetary cycle (engagement, liquidation, mandatement, paiement) and implement in at least six ministries, including the Ministries of Basic Education and Health.

Improve centralization procedures and consolidation of all balances of the principal treasury offices by the Central Treasury Accounting Office (ACCT) through the formation of a consolidation unit, constituting a step toward the establishment of general balance sheet statements and of reliable opening and closing accounting balances.

A2. Prepare biannual reports on education and health sector activities at the central and decentralized level, including

(i) budgetary allocation and expenditure execution; and

(ii) physical achievements.

B. Establish public and transparent information systern for granting licenses (beneficiary list, geographical zone, and amount) in the mining, forestry, and fishing sectors, and publish the beneficiary list biannually.
Completed Since 2000 , the budget monitoring system has been operational for the Ministries of Health and Basic Education. It has been expanded in early 2003 to cover the Ministries of Public Works, Rural Development, Forestry and Water, and Justice. From July onward, the system also covers grants, loans, and Counterpart Funds (FCV).

Completed In 2001, all the 22 principal treasury offices were computerized; initial and closing balances were established for the period 1993-98. The anmual balances for 1999 and 2000 were established before end 2002, the one for 2001 was completed in Jume 2003, and the one for 2002 is on track to be established.

Completed

Quarterly budgetary allocation reports are being regularly compiled.

Completed The first physical outcome report was completed in December 2003.

Partially The information on licenses was advertised in Completed September 2003 for the mining sector in the local press and on the website of the Mining Cadastre Office, in October 2003 and December 2003 for the fishing sector, and in April 2003 for the forestry sector. However, except for the fishing sector, the amounts of the indivictual fees were not published as indicated in the President's Memorandum and Reconmendations (P-7420MAG) at the decision point. The list, inchuding the amounts of individual fees in mining and forestry, is expected to be published in the next three months. 


\begin{tabular}{c|c|c}
\hline Triggers & Status & Comments \\
\hline
\end{tabular}

C1. Expand teacher availability in rural areas by:

(i) formalizing and implementing new financial incentives for teachers to serve in rural public primary schools; and

(i) recnuiting at least 3,500 new teachers from 2000 onward for public primary schools and deploying at least 60 percent of them to schools with contractual teachers or with a ratio of pupils to publicly paid teachers in excess of 50 .

C2. Make operational the generic essential drug supply system of district pharmacies, including the start-up of generic drug supply for hospital outpatients.

C3. Cover current road maintenance needs (100 percent) through the Road Maintenance Fund, of which at Jeast 10 percent for rural roads.
Completed The system of financial incentive has been established.

Partially completed

In 2001, 3,500 teachers were recruited and assigned to rural areas. However, only 49 percent are present in remote areas, and the ratio of teachers to students needs to be improved. The Ministry of Education is updating these numbers, based on the results of a recent survey.

Completed

The central agency (SALAMA) is fully operational and its anmual gross revenues have been increasing for the last three years. In 2003, a protocol of agreement was signed by the government and SALAMA regarding the recapitalization of the latter.

Completed The functioning of the Road Maintenance Fund (FER) was unsatisfactory in 2001, with irregular government transfers. For 2002, 10 percent of the Fund were used for rural roads. For 2003, 10 percent of the Fund were allocated for rural roads. 
Madagascar: Status of Actions to Strengthen Tracking of Poverty-Reducing Public Spending

\begin{tabular}{lllll}
\hline Actions & $\begin{array}{l}\text { Timing } \\
(\mathrm{S} / \mathrm{M})^{1}\end{array}$ & $\begin{array}{l}\text { Status } \\
(\mathrm{FL} / \mathrm{L} / / \\
\text { NS) }\end{array}$ & $\begin{array}{l}\text { Date } \\
\text { Achieved }\end{array}$ & Comments \\
\hline
\end{tabular}

\section{Actions to strengthen budget formulation}

Define budget aggregates along several poverty categories ("high, medium, or low poverty reduction relevance") of the existing functional budget classification systems, using as much as possible the information on the incidence of expenditures by population poverty groups.

Derive baseline of budget distribution by poverty classification for most recent year possible.

Migrate Tamatave system from pilot phase to fully operational system based on Oracle, and implement system in three main Treasuries by mid 2005.

Draft plan on how the medium-term orientation of the budget can be improved. A multiyear expenditure forecast should be derived from a macroeconomic projection model, be consistent with sectoral policies, and include both current and capital expenditures.

Introduce, in stages, a medium-term expenditure planning framework.

Development of coherent capacity building plan for the Ministry of Finance.
$\mathrm{S}$

NS

S NS

M

II

S Il

M II

The government has developed program budgets for the main spending ministries.

M II

Continue education of higher level staff (inspecteurs de trésor) at the Malagasy Institute for Technical Planning. 


\begin{tabular}{l|l|l|l|l}
\hline Actions & $\begin{array}{l}\text { Timing } \\
(\mathbf{S} / \mathbf{M})^{1}\end{array}$ & $\begin{array}{l}\text { Status } \\
(\mathbf{F L} / \mathbf{I} / 1 / 2 \\
\text { NS })^{2}\end{array}$ & $\begin{array}{l}\text { Date } \\
\text { Achieved }\end{array}$ & Comments \\
\hline
\end{tabular}

\section{Actions to strengthen budget execution}

Design and implerment a monitoring system for the entire budgetary cycle (engagement, liquidation, mandatement, paiement) for at least six ministries of special importance to poverty reduction, including the Ministries of Basic Education and Health.

Prepare biannual reports on education and health sector activities at the central and decentralized level, including (i) budgetary allocation and expenditure execution; and (ii) physical achievements.

Install and use ASYCUDA ++ software for customs operations in at least six priority sites.

In the context of the riew Organic law on Public Finances to be presented to the Parliament by end-2003, simplify the budget execution structure by combining the functions of the gestionnaires de credit and the sousordonnateurs in the ministries.

Implement a quantitative study showing what factors are responsib]e for the slow implementation of the execution of the investment budget in the social sectors.

Review, update, and simplify the rules and regulations for the complete budgetary cycle (public procurement, inventory assessment, and nomenclature of documentary evidence) based on diagnostic studies, including the Country Fiduciary Assessmemt.

Transform the auditor general office into an independent court (Cour des Comples).

\section{Actions to strengthen budget reporting}

Publish regularly in the official government journal all virement des credits (transfers between credit lines carried out after the budget law is passed by the parliament).
S II

System implemented for the six ministries.

$\mathrm{S}$

1 December 2003

M II

The government is about to sign a contract for a new customs system.

S NS

Organic Law on Public Finance submitted to conseil de gouvernement.
S II

$\mathrm{S}$ NS

S NS

\begin{abstract}
A committee to follow up and review the public procurement reforms is established.
\end{abstract}




\begin{tabular}{|c|c|c|c|c|}
\hline Actions & Timing & $\begin{array}{l}\text { Status } \\
\text { (FU/IV } \\
\text { NS) }^{2} \\
\end{array}$ & $\begin{array}{l}\text { Date } \\
\text { Achieved }\end{array}$ & Comments \\
\hline $\begin{array}{l}\text { Improve the centralization procecures and } \\
\text { consolidation of all balances of the principal } \\
\text { treasurers by the central treasury accounting } \\
\text { office (ACCT) through the formation of a } \\
\text { consolidation and audit unit, constituting a step } \\
\text { toward the establishment of general balance } \\
\text { sheet statements and of reliable opening and } \\
\text { closing accounting balances. }\end{array}$ & $S$ & II & & $\begin{array}{l}\text { The closing balance } \\
\text { for } 2001 \text { was prepared } \\
\text { in June } 2003 \text { and the } \\
\text { one for } 2002 \text { is on } \\
\text { track to be established. }\end{array}$ \\
\hline $\begin{array}{l}\text { Strengthen capacity and efficiency of control } \\
\text { organs based on recommendations of } \\
\text { government repoit. }\end{array}$ & $S$ & II & & $\begin{array}{l}\text { HIPC funds used to } \\
\text { strengthen control } \\
\text { organs. }\end{array}$ \\
\hline $\begin{array}{l}\text { Install, by end } 2003 \text {, the treasury computer } \\
\text { system for all main } 22 \text { treasury offices. }\end{array}$ & $S$ & $\mathrm{FI}$ & $11 / 01$ & \\
\hline $\begin{array}{l}\text { Prepare the } 2000 \text { and } 2001 \text { budgetary execution } \\
\text { laws (lai de règlement) during } 2003 \text {. }\end{array}$ & $S$ & II & & $\begin{array}{l}\text { The documents to be } \\
\text { submitted to the Cour } \\
\text { des Comptes and then } \\
\text { to the parliament. }\end{array}$ \\
\hline
\end{tabular}

${ }^{1} \mathrm{~S}=$ Short-term action; $\mathrm{M}=$ Medium-term action.

${ }^{2} \mathrm{FI}=$ Fulty implemented; 11 = Implementation initiated ; NS = Not started. 
Antananarivo, March 2, 2004

Mr. Horst Köhler

Managing Director

International Monetary Fund

Washington, DC 20431

Dear Mr. Köhler:

The attached memorandum on economic and financial policies describes the policies and measures that the Government of Madagascar has adopted and pursued since the beginning of 2003, and explains in detail Madagascar's economic and financial policies for 2004. All the performance criteria for end-June 2003 were observed. The government requests that this document serve as the basis for the Fund's completion of the fourth review under the Poverty Reduction and Growth Facility (PRGF) arrangement approved on March 1, 2001.

The Government of Madagascar requests rephasing of the remaining two disbursements, for July 2004 and February 2005, based on performance criteria for endMarch and end-September 2004, and on completion of the fifth and sixth reviews, respectively. It also requests that the PRGF arrangement be extended to March 1, 2005 in order to allow the final disbursement. Further, it requests additional interim assistance under the enhanced HIPC Initiative for the period from March 19 through July 2004, as Madagascar expects to reach the completion point in August 2004. The fifth review of the PRGF arrangement is expected to be completed by July 2004.

The Government of Madagascar believes that the policies described in the attached memorandum are sufficient to achieve the program objectives but is prepared to take any additional steps that may become necessary to achieve these objectives. The government will continue to provide the Fund with any information deemed necessary to monitor program implementation. The government will consult with the staff of the IMF before implementing policies that may affect the program's objectives or assumptions.

Sincerely yours,

$/ \mathrm{s} /$

Benjamin Andriamparany Radavidson

Minister of Economy, Finance, and the Budget is/

Gaston Ravelojaona

Governor

Central Bank of Madagascar

Attachment 


\section{MADAGASCAR \\ Memorandum on Economic and Financial Policies for 2004}

March 2, 2004

\section{INTRODUCTION}

1. In mid-2002, the government of Madagascar embarked on a medium-term program of poverty reduction and growth, as detailed in its full poverty reduction strategy paper (PRSP) that was adopted by the government in July 2003. The program seeks to achieve its objectives by creating an environment conducive to the development of the private sector, the engine of growth, opening the economy and attracting foreign investment, reorient government investment towards poverty reduction goals, and enhancing efficiency and governance in the public sector. Important measures have been taken toward these goals. An anticorruption commission has been established and the drafting of an anticorruption strategy has begun, capital goods imports have been exempted from all taxes and tariffs for a twoyear period, tax and customs administrations have been reinforced, the legal framework for landownership has been revised with a view to allowing foreign ownership of land, and investment in education, health, transport infrastructure, and rural development has been given priority.

2. The PRSP was elaborated through a number of workshops with the participation of ministries, the private sector, elected members of civil society, nongovernmental organizations (NGOs), and other partners. There were broad consultations at the regional and national levels on sectoral issues (urban and rural poverty, AIDS, gender, environment, education, health, and governance). The PRSP has been validated by the highest institutions of the country: the Council of the Government and the National Assembly. The political parties also actively participated in the national workshop in March 2003. Information and communication campaigns regarding the PRSP were conducted in all regions. The consultation process will be strengthened during the monitoring of the implementation of the action plans and the evaluation of the impact of the strategy on poverty. The PRSP was submitted to the IMF and the World Bank in July 2003.

\section{Recent Economic Developments and Program IMPlementation}

3. The implementation of the Poverty Reduction and Growth Facility (PRGF)-supported program remained on track during the first six months of 2003. All quantitative and structural performance criteria for end-June 2003 were met, macroeconomic stability was ensured, and poverty reduction policies were pursued. The structural benchmark on the preparation of monthly treasury balance sheets up to end-200l was not met, although annual balance sheets were prepared up to end-2001. The treasury will prepare quarterly balance sheets beginning in 2004 , to be followed by monthly balance sheets at a later stage. 
4. Preliminary estimates through September 2003 indicate that growth has resumed and inflation has been subdued. The agriculture, tourism, construction, and transport sectors are showing signs of recovery. Port activity during the first rine months of 2003 was 82.1 percent higher than during the same period of 2002. Consumption of petroleum products increased by 42 percent during the same period, while employment in the export processing zone (EPZ) tripled to 80,000 , which is still below the level of 100,000 recorded prior to the crisis.

5. The overall fiscal deficit through end-September 2003 (estimated at 3.2 percent of GDP, on a commitment basis, including grants) was less favorable than expected under the program. Tax revenues exceeded the program projection by FMG 77 billion to reach FMG 2,557 bilion, but expenditure exceeded the program level by FMG 121 billion. The higher revenues resulted from a faster pace of economic growth, as well as a stronger effort to collect unpaid taxes and improve efficiency at the customs and tax administrations. In August 2003, the Parliament adopted a tax relief law (loi de détaxation), which exempted imports of capital goods and selected other products from import taxes. This law came into effect on September 1. The net effect of this measure on 2003 tax receipts is estimated to have amounted to 0.24 percent of GDP.

6. Unforeseen circumstances have led to FMG 283 billion in extrabudgetary expenditures in 2003 ( 0.8 percent of GDP). The extrabudgetary expenditures include the following: FMG 72 billion of additional indemnities for the military; FMG 135 billion for additional scholarships for university students and overtime pay for teachers; FMG 14 billion paid to Lufthansa as management fees of Air Madagascar; and FMG 62 billion for several other expenditures. Net domestic arrears of FMG 145 billion (compared with a net payment of FMG 341.6 billion programmed) have accumulated, owing to the large size of investment expenditure for which payment orders have not yet been issued.

7. A new mechanism for monitoring expenditure commitments on a monthly basis was installed in the General Directorate of the Commitment Control Office (CDE). Likewise, a cash-flow management system is being used by the treasury for payment forecasting. However, the link between these two systems will be established only once, as expected, the computerized integrated system of public finance has been extended to all major locations in the country.

8. Monetary conditions during the first six months of 2003 were tighter than envisaged under the program. Reserve money declined by 14.2 percent, compared with a programmed target of 7.4 percent, while broad money (M3) declined by 2.9 percent against a programmed increase of 7.6 percent. Commercial banks' weighted-average lending rates remained high in real terms, and credit to the private sector grew by 1.2 percent, compared with a program target of 11.2 percent. These developments reflected mainly the government's repayment of the statutory advances from the central bank through the sale of treasury bills to bank and nonbank sectors. Domestic liquidity grew during the second half of 2003, as credit to government and crop financing picked up. To facilitate the deepening of the securities market, the government began issuing two- and three-year securities on tap in August 2003. 
9. The external current account deficit, including transfers, reached SDR 99 million, in the first half of 2003. Disbursements of budgetary assistance by donors, including the World Bank and the European Union, amounted to SDR 88.1 million during the first nine months of the year, compared with a programmed amount of SDR 111.3 million. The central bank's net external reserves increased from SDR 266.6 million at end-2002 to SDR 296.1 million at end-September 2003. This increase reffects foreign aid inflows, including debt relief under the Initiative for Heavily Indebted Poor Countries (HIPC Initiative), albeit largely offset by a rise in imports.

10. The government continued to implement a flexible exchange rate policy, but periodic interventions of the Central Bank of Madagascar (BCM) in the foreign exchange market mitigated the appreciation of the Malagasy franc against the U.S. dollar during the first nine months. The exchange rate at end-September stood at FMG 6,053.9 against the U.S. dollar, reflecting an appreciation of 5.9 percent from end-December 2002. The exchange rate relative to the euro depreciated by 4.9 percent during the same period.

11. Progress was made in improving efficiency and combating fraud and corruption in the tax and customs departments. The joint private/public sector committee overseeing these efforts has begun to meet on a weekly basis to review the progress of the action plan for reforms. The procedures for inspection of imports were simplified. The tax department has also strengthened its tax collection units, especially as regards collections from large enterprises.

12. The new currency, the ariary, was issued on August 1,2003, and it is circulating simultaneously with the Malagasy franc. The introduction of the new currency has been orderly, with no impact on prices and output. A formal notification of the currency change has been sent to the Fund (Attachment III).

\section{Prospects for Achieving the Macroeconomic ObJectives For 2003}

13. Given the rebound in economic activity observed in the first three quarters of 2003 and the likely boost in investment for the rest of the year (as a response to the tax relief measures), real GDP growth rate is estimated at 9.6 percent, which is more than the 6 percent growth programmed for the year. Inflationary pressures are expected to remain subdued in 2003 , with the consumer price index (CPI) inflation rate falling to 0.3 percent at endDecember 2003 , on a year-on-year basis, substantially below the program target of 7 percent.

14. Despite an improvement in the terms of trade of about 6.7 percent (compared with a projected increase of 0.7 percent), mainly due to higher vanilla export prices and lower oil import prices, the external outlook for 2003 is expected to deteriorate, compared with projections in March 2003, because of a shortfall in foreign aid and an increase in imports induced by the recent tax relief measures. The balance of payments is projected to record an overall deficit of SDR 26.5 million, reflecting largely the widening of the external current account deficit, including official transfers, to SDR 193.4 million, compared with the SDR 168.6 million envisaged under the program. Export receipts will be lower than expected 
on account of lower exports of EPZ and vanilla products. Import payments are projected to increase by 51.3 percent-compared with the forecast in March 2003 of 45 percent-due to higher food and capital goods imports. The improved external terms of trade outlook will be more than offset by shortfalls of budgetary loans from the African Development Bank (AfDB), amounting to SDR 18 million. As a result, the BCM's net official foreign reserves are now expected to increase by SDR 14 million, compared with the SDR 40 million projected in March 2003.

15. Regarding the attainment of the goverament's fiscal objectives in 2003, the overall budget deficit (on a cash basis, including grants) is expected to be contained at 4.6 percent of GDP. Bank financing of the budget deficit will be limited to 0.7 percent of GDP. By endJanuary 2004, the Government recovered FMG 50 billion owed by oil companies on account of their takeover of the liquidated state oil company, SOLIMA.

16. Important efforts were made to collect taxes, in particular on dividends and interest payments on loans, in order to achieve the objectives for 2003 . The following measures were implemented in order to preserve the integrity of the program and to contain the public deficit: (i) the closing date for budgetary commitments was moved forward to October 30 , from November 30, and (ii) the supplementary period for payments beyond the end of the year was eliminated.

\section{Program Ob.jectives and Challenges for 2004}

\section{A. Macroeconomic Framework for 2004}

17. The government has set the following macroeconomic objectives for 2004: real GDP growth of 6 percent; an inflation rate, as measured by the CPI, below 5 percent on year-onyear basis by end-December 2004; and an increase in the central bank's gross official reserves to SDR 411.9 million (equivalent to 3.8 months of projected imports). In line with the inflation and GDP targets, broad money (M3) growth will be contained at 12 percent during the year. To achieve this objective, the overall fiscal deficit (on commitments basis, including grants and the cost of structural reforms) will be limited to 2.8 percent of GDP, the ratio of the cash deficit (excluding grants) to GDP will be 8.7 percent (reflecting a more ambitious public investment program), and the primary current surplus will rise from 0.7 percent of GDP in 2003 to 2.3 percent of GDP in 2004 . The government will make net repayments to the banking system during 2004 .

\section{B. Fiscal Policy and Reform}

18. As indicated below, efforts will be made to increase government revenue by broadening the tax base, rationalizing the tax and tariff structure, and increasing tax collection (in particular through administrative reforms). Public expenditure control and government cash-flow maxagement will be strengthened in order to enhance budget execution, improve the quality of public expenditure, facilitate the monitoring and reduction of arrears (both at the treasury and ministry levels), and ensure that expenditure related to poverty reduction is implemented as planned. 


\section{4 revenue measures}

19. Strong administrative measures will be taken to improve the recovery of tax and customs revenues. Measures will be taken at the customs administration to recover an additional FMG 50 billion. These measures include the (i) auctioning of merchandises not retrieved or not declared within the statutory deadline; (ii) acceleration of the recovery of arrears in dispute; and (ii) installation of two more one-stop counters (guichets uniques) in Antsiranana and Toliary (those of Toamasina and Mahajanga are already in operation). Regarding domestic taxes, the acceleration and strengthening of legal proceedings will allow the collection of FMG 30 billion in 2004, a significant part of the total amount in dispute.

20. The 2004 budget includes measures (listed in Table 3) aimed at reversing the proliferation of ad hoc tax exemptions and introduces a comprehensive overhaul of the tax system based on the recommendations of the July 2003 IMF tax policy mission. These measures aim at broadening the revenue base and simplifying the tax system. They are expected to increase tax receipts by FMG 274 billion, partially compensating for the impact of the August tax relief measures on 2004 tax revenue, which is estimated to amount to FMG 395 billion. As a result of these measures and tax recovery efforts, the tax-to-GDP ratio will rise to 11.2 percent in 2004 (including collection of arrears) from an estimated 10 percent in 2003 . The 2004 budget law has rescinded the prohibition introduced in 2003

on the reimbursement of value-added tax (VAT) paid by exporters and investors.

21. In September 2005, the temporary exonerations-scheduled for a two-year period starting in September 2003 and adopted during the second semester of 2003-will not be renewed. Moreover, the government will nat grant exemptions beyond those included in the 2004 budget law. The VAT will remain the main tax for mobilizing domestic revenue, and measures will be taken to maintain its integrity. Work will be undertaken in 2004 to prepare for the implementation of the VAT on bank margins in 2005, which will replace the current system that taxes interest on bank loans. Moreover, it is envisaged that the customs duty and import tax will be collapsed into one tax in 2005. In that year, the tax revenue-to-GDP ratio will be maintained at a minimum of 11.6 percent.

\section{4 expenditure measures}

22. On the expenditure side, the 2004 budget includes (i) an increase in capital expenditure to FMG 3,076 billion in 2004, from FMG 1,969 billion in 2003, to be financed mainly by foreign loans and grants, with local counterpart funds amounting to about FMG 914 billion, or 30 percent of total financing; (ii) a rise in investment expenditure on the priority sectors of health (of 10 percent to FMG 215 billion), education (of 250 percent to FMG 314 billion), transport (of 23 percent to FMG 1,063 billion), and rural development (of 63 percent to FMG 324 billion); (iii) an increase in poverty-related expenditures from 1 percent of GDP in 2003 to 3 percent of GDP in 2004; (iv) the cost of the securitization of government debt to the central bank (see para. 36 below); (v) the recurrent cost of investment projects (especially the road network, reflected in transfers to the Road Maintenance Fund amounting to FMG 58.1 billion); and (vi) realistic estimates of nondiscretionary expenditure 
commitments, such as the fees to be paid for preshipment inspection of imports, estimated at FMG 22 billion in 2004. Government expenditure will be cut and administrative measures will be taken should the revenue projections prove optimistic.

23. The 2004 budget allows the government to begin paying contingency liabilities to the sugar planters (FMG 14 billion) and the telecommunications company, TELMA (FMG 130 billion), and to settle its arrears to the utility company, JIRAMA (FMG 62 billion). It also provides for the repayment of the debt of the liquidated oil company, SOLIMA, to the central bank.

24. The wage bill will be contained at 5.1 percent of GDP in 2004. No wage increase is expected. The wage bill includes new recruitments in the areas of health $(2,300)$, education $(5,500)$, justice (75), and security (500). In addition, civil servants will be encouraged through compensation to go on early retirement, consistent with the new law on civil service and public administration reform.

\section{Balance of Payments and External Debt}

25. The program is asmed at containing the external current account deficit, including grants, at about 5.4 percent of GDP, compared with 4.9 percent of GDP in 2003, and projects an increase in the central bank's net official reserves of approximately SDR 114 million. Exports are expected to increase by roughly 8.4 percent in SDR terms, owing to a rebound in EPZ activity, and despite an anticipated decrease in the export volume of vanilla. The rise in imports in SDR terms could amount to 16.3 percent, reflecting increased purchases of capital goods and petroleum products. Gross external aid, including debt relief, is expected to total SDR 414 million.

\section{Trade reform and the medium-term strategy}

26. The government will continue its policies aimed at integrating the Malagasy economy into the regional and global economies, thereby enabling Madagascar to regain the market share it lost because of the 2002 crisis. In addition to the measures included in the 2004 budget law to simplify the tariff structure (see para, 21 above), the government will aim at diversifying the export base within the EPZ, while promoting the growth of exports from outside that zone. The government will also implement a strategy aimed at reducing transaction costs, which are high owing to inadequate industrial infrastructure and inefficiencies of customs administration. Furthermore, to ensure that exports of textile products contimue to benefit from the African Growth and Opportunity Act (AGOA), Madagascar's cotton sector must be restructured, in particular by privatizing the major components of the supply chain while attracting strategic investors.

27. On regional integration, the government has taken the following steps in preparation for the launching of the customs union of the Common Market for Eastern and Southern Africa (COMESA) in December 2004 (as stipulated in Articles 45 and 47 of the COMESA Treaty): (i) completion of an ongoing study on the impact of Madagascar's membership in the future customs union; (ii) completion of an ongoing study on the creation of a regional 
stock exchange; (iii) membership of Madagascar in the Agency for Trade Insurance in Africa; (iv) membership in COMESA's Development Fund; (v) official announcement of Madagascar's signing of COMESA's protocol on the free movements of factors in 2004; (vi) development of a program aimed at improving infrastructure in order to promote external trade; and (vii) continuation of ongoing study on the opportunities and implications of Madagascar's adoption of the Organization for the Harmonization of Business Law (OHADA) Treaty. In addition, the government has adopted an action plan, with a view to carrying out the recommendations of the interministerial task force in charge of assessing, inter alia, the consequences of Madagascar's concurrent membership in regional trading blocs, and the consistency of these memberships with World Trade Organization (WTO) rules. In 2004, this action plan includes the following: (i) reform of the competitiveness law; (ii) establishment of the list of products eligible for preferential treatment based on the common tariff nomenclature of the future customs union (COMESA); (iii) a study on the impact of the adoption of COMESA's common external tariff (TEC) on the competitiveness of its main economic sectors; and (iv) the harmonization of customs' rules and procedures. This action plan takes into account the framework for negotiations of the Economic Partnership Agreement with the European Union scheduled to go into effect in 2008. COMESA's tariff code will be prepared by an expert. The government will adopt the code before the start of the customs union in 2004.

28. Regarding the vanilla industry, the government is determined to stop the decline in exports. The decline observed in the 2003 recorded exports was due to theft and degradation in the quality of vanilla, especially during the 2002 crisis. As a result, the government will take the following measures: (i) secure the production and distribution of vanilla through an increase in the number of security personnel in the production areas; (ii) restructure the vanilla supply chain by delimiting the role and responsibility of actors in the sector; (iii) modernize the sector through information, education, and communication campaigns and implementation of appropriate regulations, consistent with the liberalization and divestiture strategy of the government. These reforms will be implemented with the help of the European Union.

\section{Exchange rate policy and exchange market reform}

29. The government intends to pursue a flexible exchange rate policy. The central bank will intervene in the foreign exchange markel only to dampen sharp swings in the exchange rate and achieve its foreign reserves objective under the PRGF-supported program. The central bank will not engage in any operations in the market except for the above considerations and the support of bona fide government or central bank obligations.

30. The progress that is being made in the establishment of a new interbank foreign exchange market would allow the exchange rate to be fully market determined. The infrastructure for efficient operations by foreign exchange dealers (e.g., dedicated phone lines for connection to Info Reuters) is being put in place, and the training of bank and BCM staff by professional foreign exchange dealers will be completed by end-February 2004 . The BCM intends to publish a code of conduct and regulations governing the operations of the new 
interbank foreign exchange market. However, the opening of the market, originally envisaged for December 2003, will be delayed to March 2004 to allow the establishment of the requisite conputer network that links all banks and the BCM. To facilitate the operations of banks in this market, the authorities will consider raising banks' intraday foreign exchange net open position limits when the market opens. The overnight limit will remain at 20 percent of capital.

31. The foreign exchange control law that was expected to be presented to the parliament by end-2003 has been delayed. This law and new exchange regulations are being drafted with assistance from the IMF. The draft law will be sent to parliament by end-October 2004. The reorganization of the BCM's Exchange Operations Directorate will be completed by endJune 2004.

\section{External debt}

32. The government has made progress in finalizing bilateral rescheduling agreements with Paris Club (PC) creditors based on the Agreed Minutes of the PC meeting of March 2001. Bilateral agreements (i) have been finalized with several countries (e.g., Spain, Austria, Switzerland, Germany, Canada, France, Israel, Italy, the United Kingdom, the United States, and the Russian Federation), (ii) are near completion with some countries (e.g., Sweden), or (iii) are experiencing significant delays (e.g., Belgium and Japan) as parties are negotiating the draft agreement. The Paris Club has now extended the consolidation period to November 30,2004 and postponed the deadline for completion of bilateral agreements to December 2003. Similar agreements have been, or are about to be, signed with a few non-Paris Club creditors, notably China, Kuwait, and Saudi Arabia. The other main non-Paris Chb creditors, Abu Dhabi, Algeria, Angola, Irac, and Libya have not yet responded to Madagascar's proposals. All external arrears vis-à-vis Russia have been cleared and confirmed as such by the Russian authorities.

33. Madagascar has received interim assistance under the HIPC Initiative from the World Bank, the AfDB, the Paris Club, the OPEC Fund, China, and the IMF. The government would like to request interim assistance in the amount of SDR 0.9 million in 2004 from the Fund. In anticipation of reaching the HIPC Initiative completion point in August 2004, contacts have been made since January 2004 with all creditors in order to reconcile external debt data at end-2003 and to update the debt sustainability analysis (DSA).

\section{Monetary Policy and Financial Sector Reform}

34. The main objective of monetary policy is to control inflation. The BCM will continue to monitor closely liquidity developments in the banking sector and take appropriate action to ensure achievement of the inflation objective. To this end, the BCM will continue to refine its procedures for monthly liquidity management. The forecasts will be used to determine the level of government securities to be sold during each auction. In case the amounts required for govermment finaricing are different from the need for absorption of liquidity, the central 
bank will engage in repurchase and reverse-repurchase operations to achieve the desired level of liquidity.

35. The legal framework for microfinance activities is being developed. The government plans to adopt a national policy in line with the PRSP and on the basis of the recent review of the sector. Measures to encourage these activities include (i) the implementation of a legal and statutory framework specific to microfinance activities for all institutions; and (ii) the professionalisation of microfinance institutions by monitoring their risks and development.

36. To improve transparency of the government's relations with the central bank, government debt to the central bank amounting to FMG 1,620 billion (of which FMG 524 billion is denominated in foreign currency) will be converted into securities. The 2004 budget law will include a provision authorizing the purchase of government securities on the primary market for this specific operation. The securities sold to the central bank will carry a market-related interest rate. The modalities of this operation will be specified in an agreement between the government and the BCM. To minimize the initial impact of this operation on the budget, the $\mathrm{BCM}$ will transfer its net income from the operation to the treasury on a timely basis. The 2004 budget law will also prohibit the government from borrowing from the central bank in foreign exchange or from holding foreign currency deposits at the central bank, unless required by donors.

37. The audit of reserves management and foreign exchange activities has begun, as recommended by the safeguards assessment report, and anti-money-laundering legislation has been enacted. The remaining safeguards requirements have been or will be carried out as follows: (i) the BCM's 2000, 2001, and 2002 financial statements were published in the official journal; the attendant audit opinions will be published in the official journal and on the central bank's website by end-March 2004; (ii) for comparison and training purposes, the BCM's 2003 financial statements will be prepared both according to official accounting system and to international accounting norms (IAN) by end-September 2004. The official statements will include explanatory notes on the differences between the official and International Accounting Standards presentations; and (iii) all BCM financial statements will be prepared on an IAN basis and continue to be audited by a reputable international accounting firm, starting with the 2005 accounts.

\section{E. Structural Reforms}

\section{Structural fiscal reforms}

38. The following measures will be taken in order to improve the monitoring of investment expenditure: (i) regular communication (every two months) to the General Directorate of Public Expenditures of all drawing on externally financed projects; (ii) regular communication by task managers of the Fiche de Centralisation Comptable (FCC), stamped by the Commitment Control Office (CDE) at the Directorate of Public Investment, to the General Directorate of Public Expenditures on the thirtieth of every month; (iii) improvement of the communication and information system linking all directorates; (iv) the strengthening 
of skills in technical and financial analyses; and (v) organization of workshops for the priority ministries, in collaboration with donors, on budget management every four months, with a view to teaching project managers how to execute their expenditures, as well as to monitor effectively project implementation. The government consulted extensively with the donor community on how to better target and track povertyrreducing spending and has approved the recommendations of the July 2003 Assessment and Action Plan. The timetable for the implementation of these recommendations was established by the public finance legislation review mission that was fielded during November 10-21, 2003.

39. The tax and customs departments will take the following actions to improve their operations: (i) improve the relationship between the tax services and taxpayers through better information and tax collection, as initiated in 2003; (ii) develop a new public relations' unit for the large enterprises at the tax department, in collaboration with the large taxpayers; (iii) strengthen tax services through computerization of 45 new centers in 2004 (compared with 30 in 2003); (iv) simplify and enhance procedures through the creation of one-stop counters, the use of selective inspections, and a shortening of the time spent at customs (to 24 hours in Toamasina and Mahajanga, and 48 hours at Ivato and in Antanimena); (v) intensify ex post controls, fight against fraud, and conduct surveillance and investigation operations at customs offices (through a better monitoring of the accounts of enterprises in the facilities at the ports); (vi) intensify the computerization of the customs services; (vii) use suggestion boxes to get feedback from taxpayers; (viii) elaborate guidelines for taxpayers and procedure manuals for customs officers; (ix) conduct training on ASYCUDA +4; and (x) create a committee to settle disputes, involving government representatives, the private sector, and the preshipment inspection company (SGS). The committee overseeing the reforms of the tax and customs departments will report on the first six months of its operations by end-March 2004, indicating its achievements, the work remaining to be done, and problems faced in the implementation of its agenda.

40. The accumulation of domestic arrears (FMG 205 billion and FMG 558 billion, on a gross basis, at end-June and end-September 2003, respectively) is due to the large increases in investment expenditures committed since June 2003. In 2004, the budget circular will require that all ministries and institutions commit expenditures in line with the resources available to them. Those who fail to comply will be given administrative sanctions.

41. The computerized, integrated public funance system was installed in Toamasina in early 2003 and in Antananarivo in June 2003. It will be gradually extended to the other provinces in 2004. The delay in the preparation of the treasury monthly accounts will be reduced to 15 days in areas where the integrated system is established. The quarterly "flash" reports on budget execution, which now cover six ministries, will be extended to all ministries in 2005 .

\section{Public enterprise reform}

42. The divestiture contract for the telecommunications company, TELMA, was signed in August 2003 and will be finalized once the current foreign shareholder sells its shares to the 
new investor. The report of a Wortd Bank-financed consultant on the financial situation and management of JRAMA was completed in December 2003. The government will implement the recommendations forthwith, including any proposal for changes in the company's tariff structure. The terder for the placement of JRAMA under a management contract has been delayed. The tender was launched before end-January 2004 and the company will be placed under new management by end-June 2004. The rehabilitation and privatization of the sugar company, SIRAMA, and the cotton company, HASYMA, have begun. The bidding process for the selection of the company that will manage SIRAMA and the purchase of HASYMA will be completed by end-June 2004 and end-March 2004, respectively.

43. The procedures for the establishment of the price of petroleum products to final consumers, which were established on a temporary basis on the liquidation of SOLIMA in 2001 , will be eliminated by end-2004. Petroleum product prices will then be allowed to move freely, reflecting market onditions.

\section{POVERTY AND SOCLAL IMPACT ANALYSES}

44. The government will undertake poverty and social impact analyses of recent and prospective policy initiatives, including the envisaged retrenchments in the public enterprise sector and the changes in tax and tariff policies intraduced in August 2003 and in the 2004 budget law.

\section{Program Monitohung}

45. The program supported by the IMF under the PRGF will be monitored through semiannual reviews, quantitative and structural performance criteria and benchmarks, indicative targets, and quarterly monitoring indicators shown in Annex (Tables 1 and 2). The following measures constitute prior actions for completing the fourth review under the PRGF arrangement: (i) submission to the Parliament of the 2004 budget law incorporating the tax policy measures presented in Table 3; (ii) collection of the full amount owed to the government by the oil companies in connection with the liquidation of SOLIMA; and (iii) launching of international tender for the placement of JIRAMA under a management contract.

\section{CONSUltation WITH THE IMF}

46. The government will consult with the staff of the IMF before implementing policies that could have an impact on the objectives or assumptions of the program. 
Table 1. Madagascar: Quantitative Performance Criteria and Indicative Targets for 2003-04 under the PRGF Arrengement

(In billions of Malagusy franes, urkess otherwise indicated) $1 /$

\begin{tabular}{|c|c|c|c|c|c|c|c|c|c|c|c|c|c|c|}
\hline & \multicolumn{10}{|c|}{2003} & \multicolumn{4}{|c|}{2004} \\
\hline & \multicolumn{2}{|c|}{$\begin{array}{c}\text { Marnch } \\
\text { lpdicative }\end{array}$} & \multicolumn{3}{|c|}{$\begin{array}{l}\text { June } \\
\text { Peffornance } \\
\text { Crituris }\end{array}$} & \multicolumn{3}{|c|}{$\begin{array}{l}\text { Septentere } \\
\text { Indifurtive }\end{array}$} & \multicolumn{2}{|c|}{ 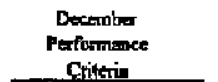 } & \multirow[t]{2}{*}{$\begin{array}{c}\text { Mareb } \\
\text { Pertímusce } \\
\text { Critaía. }\end{array}$} & \multirow{2}{*}{ 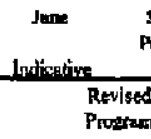 } & $\begin{array}{l}\text { Sepptember } \\
\text { Performance } \\
\text { Cilteris: }\end{array}$ & \multirow[t]{2}{*}{$\begin{array}{l}\text { Decembse } \\
\text { Indirative }\end{array}$} \\
\hline & mogram & Aentil & Program & Adfueled & Actued & Progtram & Adjusted & Extimutra & Pragantin & Projertion & & & & \\
\hline \multicolumn{15}{|l|}{ 1. Quantitative perfommance criteria } \\
\hline (a) Ceiling on attmal areass (ln milliors of SDRs) 27 & 0,0 & 0.0 & 0.0 & & 0.0 & 0.0 & & 0.0 & 0,0 & 0.0 & 0.0 & 0.0 & 0.0 & 0.0 \\
\hline & 59,4 & 96.8 & -16.1 & -274.6 & 211.4 & 73.9 & .185 .0 & 299.5 & At9.0 & 160.5 & 221.8 & 80.9 & 941.3 & 80.8 \\
\hline 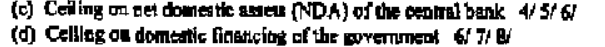 & +48.3 & -189.6 & -114.7 & 143.7 & -619.8 & $-\mid \$ 2.3$ & 106.5 & -2326 & -465.2 & .24 .5 & 23.3 & 2490 & $-\$ 61.0$ & 455.3 \\
\hline 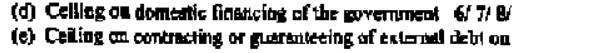 & . 103.8 & .3979 & .142 .0 & 950 & -66.7 & -116.9 & 双. & 315.3 & $-108,8$ & 512.6 & -83.3 & 108.7 & .65 .1 & -315.1 \\
\hline nonconcasinges terjus 9 & so & 0.0 & $\infty .0$ & & 0,0 & 0.0 & & 0.0 & 0.0 & 0.0 & 0.0 & 0.0 & 0.0 & 0.0 \\
\hline (A) Foor on tax reverwe & 759.0 & 879.5 & $1,700.0$ & & $1,760.0$ & $2,480.0$ & & $2,557.1$ & $3,509.0$ & $3, \$ 89.3$ & $1,010.5$ & $2,164,1$ & $3,120.3$ & $1,210,0$ \\
\hline 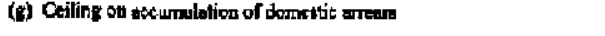 & & & & & & & & - & $\ldots$ & ... & an & 0.0 & 0.0 & 0,0 \\
\hline \multicolumn{15}{|l|}{ II. Indicmive urgets } \\
\hline (h) Cefling on resepve money & -464.4 & -464.4 & -263.0 & & .502 .7 & .236 .4 & & .27 .2 & -165.0 & 139.0 & 236.3 & 320.9 & 371.7 & 405.6 \\
\hline (i) Cesiling on broed maney (induding foreizn currency deposita) (M) & 41.8 & 43.8 & 605.0 & & .150 .5 & 817.3 & & 380.2 & $1,063.0$ & 945.2 & 617.6 & 838.1 & 970.5 & 1,056.7 \\
\hline (j) Floor on mreass paytronts & 198.3 & 261.0 & 290,0 & & 176.7 & 341.6 & & -145.0 & 341.6 & 256.6 & $1+0,8$ & 175.3 & 3020 & 347.3 \\
\hline 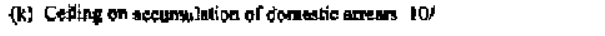 & 0.0 & 0.0 & 0.0 & & 0.0 & 0.0 & & 0.0 & 0.0 & 0.0 & & & & \\
\hline \multicolumn{15}{|l|}{ III. Menorandum ikms: } \\
\hline 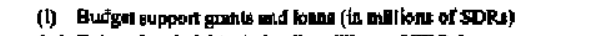 & 42.7 & & $\$ 8.3$ & & 63.7 & 311.3 & & 98.1 & 123.3 & 100.0 & 1800 & 30.1 & 119.7 & 141.2 \\
\hline 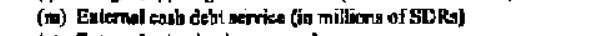 & 6.2 & 8.0 & 18.2 & & 18.3 & 253 & & 25.2 & 40.9 & .41 .9 & 8.2 & 24.9 & 33.6 & 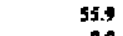 \\
\hline (n) Exletsal privatizution procesd & 0.0 & 0.6 & 21.4 & & 0.0 & 73.2 & & 0.0 & 83.5 & 0.0 & v.a & 0,0 & 0.0 & 0.0 \\
\hline (o) Total privalization prucereds. & 0.0 & 4.4 & 21,4 & & 27.9 & 33.2 & & 27.9 & 83.5 & 72.3 & 2.2 & 23.4 & 46.0 & 72.8 \\
\hline (p) External privatization reloted costs & D.0 & 0.0 & 0.0 & & 0.0 & 0,0 & & 0.0 & 0.0 & 0.0 & 0.0 & 0.0 & 0.0 & 0.0 \\
\hline
\end{tabular}

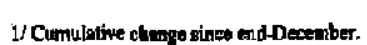

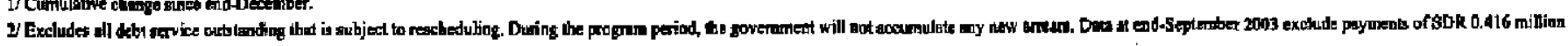

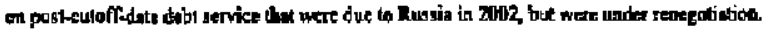

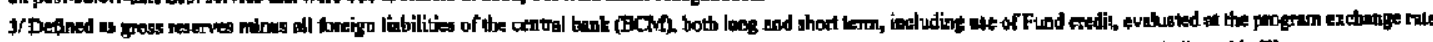

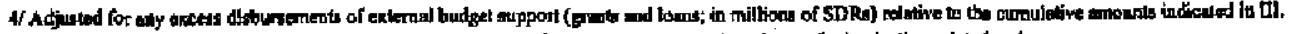

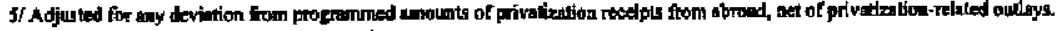

15. Adulued for the inpect of enchange rnte chinges.

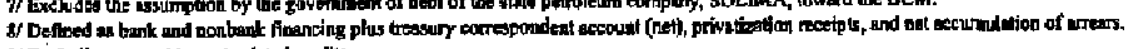

9/ Exichiding normal impott-reloted erodis.

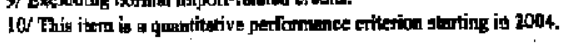


Table 2. Structural Performance Criteria and Benchmarks for the Fitth Review of the PRGF-Supported Program

\begin{tabular}{|c|c|c|}
\hline & Timing & Status \\
\hline \multicolumn{3}{|l|}{ Structural performance criteria } \\
\hline Submission of draft budget execution laws for 2000 and 2001 to the Audit Court. & By end-December 2003 & Done $1 /$ \\
\hline Completion of the bidding process for the purchase of HASYMA. & By end-March 2004 & \\
\hline $\begin{array}{l}\text { Completion of the bidding process for the selection of the company that will manage } \\
\text { SIRAMA. }\end{array}$ & By end-June 2004 & \\
\hline $\begin{array}{l}\text { No tax or tariff exemptions will be granted beyond those specified in the } 2004 \text { budget } \\
\text { law. }\end{array}$ & Continuous & \\
\hline \multicolumn{3}{|l|}{ Structural benchmarks } \\
\hline $\begin{array}{l}\text { Publication of the BCM's } 2001,2002 \text {, and subsequent financial statements and } \\
\text { attendant audit opinions in the Journal Officiel, and/or other official outlets and the } \\
\text { BCM website. }\end{array}$ & By end-March 2004 & \\
\hline $\begin{array}{l}\text { Replacement of the existing system of exchange rate determination by a continuous } \\
\text { interbank trading system. }\end{array}$ & By end-March 2004 & \\
\hline
\end{tabular}

1/ This PC was established at the completion of the third review. 
Table 3. Fiscal Measures Included in the 2004 Finance Law

Corporate profit tax (IBS): substitution of investment deductions by a permanent degressive amortization system at a single rate of 30 percent for all equipment goods.

Tax on capital income (IRCM): reduction from 25 percent to 20 percent.

Tax on transfers abroad (TFT): reduction from 15 percent to 10 percent.

Elimination of some registration taxes (to promote business).

Elimination of income tax insiallments at customs for registered taxpayers.

Elimination of $\operatorname{tax}$ on incorporation of reserves to capital.

Iricome tax [corporate profit tax (IBS) and nonwage personal income tax (IRNS)]:

elimination of deductions for indirectly productive investments.

Increase excise tax on cigarettes from 70 percent to 80 percent.

Increase the tax on tobacco.

Value-added tax (VA'T) and transaction tax (TST): taxation of ground transportation of passengers and taxation of sale of agricultural consumer goods, except for rice.

Personal wage income tax (TRSA):

- reduce IRSA in line with corporate profit tax (IBS) or nonwage personal income tax (IRNS);

- eliminate deductions for savings deposits at the National Savings Bank (CEM); and

- combine into four categories (minimum tax on monthly revenues less than FMG 250,000; 5 percent on monthly revenues between FMG 250,001 and FMG 500,000; 15 percent on monthly revenues between FMG 500,001 and FMG 1,500,000; and 30 percent on monthly revenues more than FMG 1,500,000).

Nonwage personal income tax (IRNS): fusion into three categories (minimum tax on annua] revenues less than FMG 2,500,000; 15 percent on annual revenues between FMG 2,500,001 and FMG 20,000,000; and 30 percent on annual revenues more than FMG 20,000,000).

Import taxes: fusion of customs duty (DD), import tax (TI), stamp duty on imports (DTI), and statistical tax on imports (TSI) into two categories (customs duty and import taxes). The sum of the two rates will amount to 5 percent, 10 percent, 20 percent, and 25 percent. 


\section{MADAGASCAR}

\section{Technical Memorandum on Monitoring the 2003-2004 Program Supported by the Arrangement under the Poverty Reduction and Growth Facility (PRGF)}

1. This technical memorandum defines the variables used to establish the quantitative performance criteria and benchmarks for the program, how they are calculated, and any adjustments deemed necessary. It also explains the monitoring variables, that is, anticipated balance of payments assistance and direct investment flows connected with the privatization of public enterprises. Unless otherwise indicated, in the case of stocks, variables for end-June 2003 , end-September 2003, and end-December 2003 are expressed as cumulative variations from December 31, 2002 onward, and in the case of flows as cumulative flows from January 1, 2003 onward. Variables for 2004 are expressed as cumulated variations from December 31, 2003 in case of stocks, and cumulated flows from January 1, 2004 onward, in the case of flows. The objectives for end-September 2003 and end-June 2004 are benchmarks; those for end-June 2003, end-March 2004, and end-September 2004 are performance criteria.

\section{QuantrTative Cruteria}

\section{A. Ceiling on External Payments Arrears}

2. This variable is expressed in terms of the stock of arrears. These arrears will consist of all overdue debt-service obligations (i.e., payments of principal and interest) arising in respect of loans contracted or guaranteed by the government or the Central Bank of Madagascar, (BCM) including unpaid penalties or interest charges associated with these arrears, excluding arrears resulting from nonpayment of debt service for which rescheduling negotiations are under way. This performance criterion should be observed on a continuous basis.

\section{B. Ceiling on Nonconcessional External Borrowing}

\section{Definition}

3. The nonaccumulation of nonconcessional debt contracted or guaranteed by the government is a performance criterion. Nonconcessional external debt is defined as having a grant element of less than 35 percent. This performance criterion applies not only to debt as defined in point No. 9 of the Guidelines on Performance Criteria with Respect to Foreign Debt (IMF Executive Board Decision No. 12274-00/85, August 24, 2000), but also to commitments contracted or guaranteed for which value has not been received. Under the program, nonconcessional debt includes fuancial leasing and any other instrument giving rights to a current financial liability, under a contractual arrangement by the government of Madagascar or guaranteed by the government of Madagascar, but it excludes debt contracted under rescheduling agreements and normal import-related credits of less than one year. 


\section{Calculation method}

4. Calculation of the degree of concessionality of new external borrowing is based on the ten-year average of the OECD's commercial interest reference rate (CIRR) for loans with maturities greater than 15 years and the six-month average CIRR for loans maturing in less than 15 years.

\section{Floor for Net Foreign Assets (NFA) of the Central Bank of Madagascar}

\section{Definition}

5. NFA are defined as the difference between gross international reserves and all foreign liabilities of the BCM, including debt to the IMF and other short- and long-term liabilities of the BCM. Gross international reserves are defined as assets in convertible currency that are readily available to, and controlled by, the BCM for financing payments inbalances, excluding assets that are pledged, collateralized, or otherwise encumbered.

\section{Calculation method}

6. For purposes of calculating this criterion, NFA must be converted into Malagasy francs (FMG) at the program exchange rate.

\section{Ceiling on Net Domestic Assets (NDA) of the Central Bank of Madagascar}

\section{Definition}

7. The BCM's NDA include net credit to the government, credit to enterprises and individuals, claims on banks, liabilities to banks in the form of central bank deposit auctions (appels d'offres négatifs), and other items net, excluding the foreign exchange adjustment item.

8. Foreign exchange deposits and credits must be converted into Malagasy francs at the program exchange rate.

\section{E. Ceiling on the Net Domestic Financing Requirements of the Central Government (CG)}

\section{Definjtion}

9. Net domestic financing requirements of the central government are defined as the sum of (a) the variation in the stock of the central government's domestic debt to the banking and nonbanking system, and variation in net debt to treasury correspondents; (b) domestic or foreign receipts from privatization operations, as defined in Section III.B of this technical memorandum; and (c) the variation in central government domestic arrears. Central government (CG) corresponds to the scope of operations of the treasury as indicated in the overall treasury operations table (opérations globales du Trésor, or OGT). The change in 
deposits of the treasury correspondents (correspondants du Trésor) is considered a component of domestic financing.

10. The variation in the stock of domestic payments arrears consists of the amount to be recommitted and net payments delays (clearings, items in process of payment, and expenditure committed but with no payment orders issued), as defined in the OGT.

11. Net bank claims consist of BCM and commercial bank claims on the CG, including auctioned treasury bills (BTAs) and other treasury bills and liabilities, net of CG deposits with the BCM and commercial banks, including foreign currency deposits. The change in net bank claims is defined to exclude the impact of exchange rate changes on net bank claims on the government.

12. Nonbank claims consist of BTAs and other treasury bills (BTs) and bonds placed with nonbank institutions and the public. The valuation of nonbank clams is based on the change in outstanding conventional treasury bills (maturities of one to five years), auctioned treasury bilis (maturities of one month to three years), and outstanding domestic government loans (Lova, Hasimbola, and Fanambina).

\section{Calculation method}

13. Net deposits in foreign exchange must be converted into Malagasy francs at the program exchange rate.

14. BTAs must be posted at their net value at time of initial issue.

\section{F. Floor on Tax Revenue}

15. Tax revenue includes not only that received by the treasury, but also suspense items, including those related to the public investment program.

\section{G. Ceiling on Accumulation of Domestic Payments Arrears}

\section{Definition}

16. Payments arrears consist of (1) all expenditure for which payment orders have been issued but have not been paid by the treasury within three months, and (2) the amount of VAT reimbursable to taxpayers which has not been reimbursed after three months. 


\section{INDiCATIVE LIMITS OR CEILINGS}

\section{A. Ceiling on Reserve Money}

\section{Definition}

17. Reserve money consists of notes and coins issued and demand deposits of commercial banks with the BCM (including both required and excess reserves).

18. Central bank deposit auctions (appels d'offres négatifs) are excluded from reserve money and are classified in NDA.

\section{B. Ceiling on Broad Money}

\section{Definition}

19. Broad money (M3) includes notes and coins in circulation, demand and time deposits with banks, including foreign currency deposits of residents, and bonds issued by banks.

\section{Calculation method}

20. Foreign currency deposits must be converted into Malagasy francs at the program exchange rate.

\section{Floor on Payments of Domestic Payments Arrears}

\section{Definition}

21. Payments of domestic arrears are equal to the variation in the stock of domestic payments arrears defined in paragraph 10 , with an opposite sign.

\section{Monitoring VARIABLES AND MEMORANDUM ITEMS}

\section{A. Projected Balance of Payments Assistance}

\section{Definition}

22. External balance of payments assistance is defined as loans and grants (nonproject) provided as structural adjustment financing and resulting in funds available to the treasury. It excludes the assistance that gives rise to counterpart funds for the treasury with a delay of longer than one year.

\section{Calculation method}

23. Financial assistance in foreign exchange must be converted into Malagasy francs at the program exchange rate. Assistance in kind must be posted when the counterpart funds are deposited with the treasury. 


\section{B. Projected Investment Flows Connected with the Privatization of Public Enterprises}

\section{Definition}

24. The cost of privatization operations is included above the line in central government operations. Apart from covering reform-related costs, gross receipts from the privatization of public enterprises (PEs) will be used to reduce outstanding domestic debt.

\section{ADJusters}

\section{A. Excess/Shortfall in Balance of Payments Assistance}

25. In the event that net external financing (balance of payments assistance less public debt service on a cash basis) exceeds the amount programmed, (a) the floor on the BCM's NFA will be adjusted upward (the adjustment will be converted at the exchange rate used in the operation); (b) the ceiling on the BCM's NDA will be adjusted downward (the adjustment will be converted into Malagasy francs at the exchange rate used in the operation); and (c) the ceiling on the net domestic financing requirements of the central government will be adjusted downward (the adjustment will be converted into Malagasy francs at the exchange rate used in the operation).

26. In the event of a shortfall in net external financing at end Jume 2003, end-September 2003, end-December 2003, end-March 2004, end-June 2004, end-September 2004, or endDecember 2004 against the programmed amount for the corresponding period of 2003 and 2004, the floors and cetlings will be adjusted as follows: by a maximum of SDR 45 million for the first half of 2003, by a maximum of SDR 55 million for the first nine months of 2003 and for the full year of 2003, by a maximum of SDR 11 million for the first quarter of 2004, by a maximum of SDR 17 million for the first half of 2004, and by a maximum of SDR 45 million for the first nine months of 2004 and for the full year of 2004, according to the following method: (a) the floor on the BCM's NFA will be adjusted downward by the amount indicated above (the adjustment will be converted at the program exchange rate); (b) the ceiling on the BCM's NDA will be adjusted upward by the same amount (the adjustment will be converted into Malagasy francs at the program exchange rate); and (c) the ceiling on the central government's net domestic financing requirements will be adjusted upward and capped at the above-mentioned maximum amount (the adjustment will be converted into Malagasy francs at the program exchange rate).

\section{B. Privatization-Related Transactions}

27. Adjustments will be made for any deviation in (a) privatization receipts; and (b) current privatization-related expenditure. The floor on net foreign assets will be adjusted upward or downward by a maximum of SDR 12 million from the programmed floor if net disbursed foreign resources from privatizations are higher or lower than programmed. The adjustment will be limited to a maximum of SDR 5 million for the periods from end2002 to end-June 2003, end-September 2003, and end-December 2003. Similarly, the BCM's 
NDA will be adjusted downward or upward (at the actual average exchange rate of the pertinent quarter). The celling on domestic government financing will be adjusted to take account of any discrepancies between actual privatization-related expenditures and those programmed (upward adjustment if expenditure exceeds the amount programmed and downward, in the opposite case, up to the difference reported).

\section{Program Exchange Rate}

28. The amounts of balance of payments assistance and debt service denominated in SDRs will be converted into Malagasy franc at the FMG/SDR exchange rate of FMG 9,108, FMG 9,240, FMG 9,372, or FMG 8,646=SDR 1 for each of the four quarters of 2003, respectively. Amounts of balance of payments assistance and debt service denominated in SDRs will be converted into Malagasy frane at the Malagasy franc/SDR exchange rate of Malagasy franc 8,904.7=SDR 1 for each of the four quarters of 2004. In 2003, corresponding amounts denominated in U.S. dollars and in euro will be converted by applying the rate of US $\$ 1.38=$ SDR 1 and US $\$ 1.09=€ 1$, and the Malagasy franc/SDR rate indicated above. In 2004, corresponding amounts denominated in U.S. dollars and in euros will be converted by applying the rate of US $\$ 1.40=S D R 1$ and US $\$ 1,14=€ 1$, and the Malagasy franc/SDR rate indicated above.

\section{Consultations with Fund Staff on the Performance Criterion FOR NFA AND THE BROAD MONEX BENCHMARK}

29. In the event that demand for money is stronger than expected and the exchange rate appreciates, the central bank should intervene on the interbank foreign exchange market (MID) to offset this appreciation, taking into account programmed limits (floor/ceiling) on the accumulation of net foreign assets and the level of broad money. Given the program limits on these criteria and benchmarks, if broad money growth since end-December 2003 exceeds 15 percent and/or if the level of net foreign assets exceeds the programmed level by more than 5 percent of broad money at end-2003, the authorities will consult Fund staff on measures to be taken in the context of exchange market and monetary policy management.

\section{INFORMaTION AND DATA To BE SUPPLIEd TO Fund STAFF}

30. The Malagasy authorities will provide Fund staff with information and data according to the schedule provided, either directly (e-mail or facsimile) or through the Fund's resident mission.

\section{The Central Bank of Madagascar will report the following statistics:}

\section{Monthly}

market results of treasury bill auctions, including the bid level, the bids accepted or rejected, and the level of interest rates; 
- data on the secondary market;

- information on monetary developments, as required by the Statistics Department of the International Monetary Fund (STA);

- monthly balances of the BCM and deposit money banks;

- bank lending by economic sector and by term;

- the banking risk situation;

- money market operations and rates;

- changes in bank liquidity on an end-of-month basis (required reserves and free reserves);

- the foreign exchange cashflow table, including foreign debt operations;

- the table of interbank foreign exchange operations on the MID; and

\section{Quarterly}

- data on foreign trade (exports and imports).

\section{The Ministry of Economy, Finance, and Budget will report the following information:}

\section{Weekly}

- weekly report of the customs and tax directors to the committee monitoring the customs and tax administration reforms.

\section{Monthly}

- OGT data on a cash and commitment basis and the related tables;

- expenditure on structural reform;

- central government revenue and expenditure, including short-term treasury on-lending;

- treasury liabilities (statutory advances and operations on the treasury bill market);

- central government capital expenditure;

- external public debt operations (debł contracted and publicly guaranteed, settlement of arrears, and operations of public enterprises) and debt service paid;

- the consumer price indices; and 
- indicators of sectoral economic activity.

32. Moreover, information on important measures adopted by the government in the economic and social areas that would have an impact on program development, changes in legislation, including laws passed by the National Assembly and new rules established by the Banking Supervision Commission (CSBF), and any other pertinent legislation will be reported to Fund staff on a timely basis for consultation or information, as appropriate.

$$
/ \mathrm{s} /
$$

Gaston Ravelojaona

Governor

Central Bank of Madagascar

Antananarivo, Madagascar
$/ \mathbf{s}^{\prime}$

Henri Bernard Razakariasa

Secretary General

Ministry of Economy, Finance, and Budget

Antananarivo, Madagascar 


\section{BANQUE CENTRALE DE MADAGASCAR

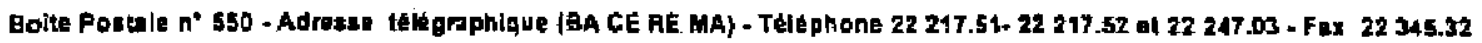

79iretion des etudes

\section{COPIE}

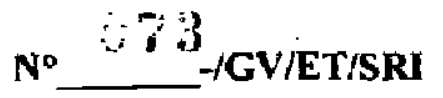

Antananarivo, le 14 îi

Dear Mr. Köhler:

In accordance with its obligations under Artizle IV, Section 2(a) of the Articles of Agreement of the Intemational Monetary Fund, Madagascar wishes to notify the Fund that, with effect from july 31, 2003, the Ariary is the monetary unit of Madagascar. For a transition period expected to last through December 2004, the Malagasy france (FMG) will continue in cireulation and will continue as the unit of account for international transactions and for transactions with the IMF. The conversion rate remains Ariary 1 = FMG 5.

Very muly vouss.

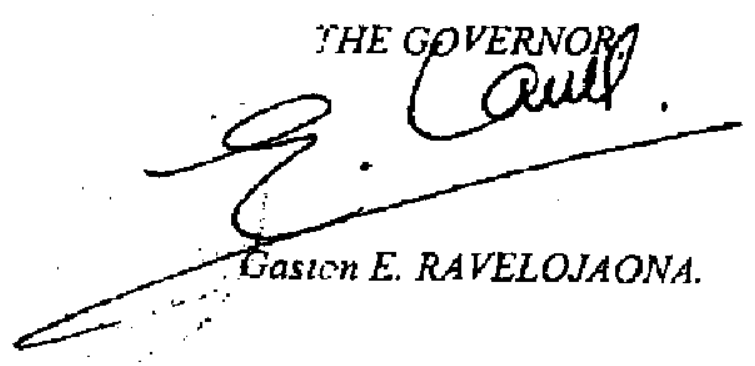

Mr. Horst KÖHLER

Managing Director

International Monetary Fund

WASHINGTON, DC 20431 (USA) 


\section{Madagascar: Relations with the Fund}

(As of December 31, 2003)

I. Membership Status: Joined 9/25/63; Article VIII

II. General Resources Account:

Quota

Fund holdings of currency

SDR Millions

122.20

122.17

0.03

$\underline{\text { SDR Millions }}$

19.27

0.02

Holdings

IV. Outstanding Purchases and Loans:

Poverty Reduction and Growth Facility

(PRGF) arrangements
SDR Millions

115.93

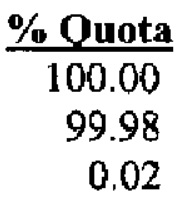

$\%$ Allacation

100.00

0.11

\section{\% Quota}

94.87

V. Financial Arrangements:

\begin{tabular}{|c|c|c|c|c|}
\hline Type & $\begin{array}{l}\text { Approval } \\
\text { Date } \\
\end{array}$ & $\begin{array}{l}\text { Expira- } \\
\text { tion } \\
\text { Date } \\
\end{array}$ & $\begin{array}{c}\text { Amount } \\
\text { Approved } \\
\text { (SDR millions) }\end{array}$ & $\begin{array}{c}\text { Amount } \\
\text { Drawn } \\
\text { (SDR millions) }\end{array}$ \\
\hline PRGF & $\overline{03 / 01 / 01}$ & $\overline{11 / 30 / 04}$ & 79.43 & 45.39 \\
\hline ESAF/PRGF & $11 / 27 / 96$ & $11 / 30 / 00$ & 81.36 & 78.68 \\
\hline ESAF & $05 / 15 / 89$ & $05 / 14 / 92$ & 76.90 & 51.27 \\
\hline
\end{tabular}

VI. Projected Payments to Fund (without HIPC Initiative Assistance)

(SDR Million; based on existing use of resources and present holdings of SDRs):

\begin{tabular}{lrrrrr} 
& \multicolumn{6}{c}{ Forthcoming } \\
\cline { 2 - 6 } & $\underline{2004}$ & $\underline{2005}$ & $\underline{2006}$ & $\underline{2007}$ & $\underline{2008}$ \\
Principal & 5.42 & 8.14 & 16.87 & 17.56 & 17.12 \\
Charge/interest & $\underline{0.87}$ & $\underline{0.84}$ & $\underline{0.77}$ & $\underline{0.68}$ & $\underline{0.60}$ \\
Total & 6.30 & 8.97 & 17.64 & 18.24 & 17.72
\end{tabular}

Projected Payments to Fund (with Board-approved HIPC Initiative Assistance) (SDR Million; based on existing use of resources and present holdings of SDRs):

\begin{tabular}{lrrrrr} 
& \multicolumn{6}{c}{ Forthcoming } \\
\cline { 2 - 6 } & $\underline{2004}$ & $\underline{2005}$ & $\underline{2006}$ & $\underline{2007}$ & $\underline{2008}$ \\
Principal & 5.42 & 8.14 & 16.87 & 17.56 & 17.12 \\
Charges/interest & $\underline{0.87}$ & $\underline{0.84}$ & $\underline{0.77}$ & $\underline{0.68}$ & $\underline{0.60}$ \\
Total & 6.30 & $\mathbf{8 . 9 7}$ & 17.64 & 18.24 & 17.72
\end{tabular}


VII. Implementation of HIPC Initiative:

$\begin{array}{lr}\text { Commitment of HIPC Initiative assistance } & \begin{array}{c}\text { Enhanced } \\ \text { framework }\end{array} \\ \text { Decision point date } & 12 / 21 / 2000 \\ \text { Assistance committed (NPV terms) } & \\ \text { Total assistance (US\$ Million) } & 814.00 \\ \text { Of which: Fund assistance (US\$ million) } & 21.60 \\ \text { (SDR equivalent in millions) } & 16.60 \\ \text { Completion point date } & \text { Floating }\end{array}$

Disbursement of IMF assistance (SDR million)

\begin{tabular}{lc} 
Assistance disbursed to the member & 5.01 \\
Interim assistance & 5.01 \\
Completion point balance & -- \\
Additional disbursement of interest income & 2 \\
Total disbursements & -- \\
\hline
\end{tabular}

\section{Safeguards Assessments:}

Under the Fund's safeguards assessment policy, the Central Bank of Madagascar (BCM) is subject to a full Stage One safeguards assessment with respect to the PRGF arrangement, which was approved on March 1, 2001 and is seheduled to expire on November 30, 2004. An off-site safeguards assessment of the BCM was completed on November 21,2001. The assessment concluded that an on-site assessment was not necessary, but identified certain weaknesses and made appropriate recommendations. These weaknesses are being addressed with the assistance of MFD, but key recommendations such as the publication of the BCM financial statements, the development and implementation of an action plan to move the BCM's existing accounting framework to International Accounting Standards (LAS), and the disclosure of the differences between the existing accounting framework and IAS remain to be implemented.

\footnotetext{
${ }^{1}$ Assistance committed under the original framework is expressed in net present value (NPV) terms at the completion point, and assistance committed under the enhanced framework is expressed in NPV terms at the decision point.

2 Under the enhanced framework, an additional disbursement is made at the completion point corresponding to interest income earned on the amount committed at the decision point but not disbursed during the intcrim period.
} 


\section{Exchange System and Exchange Rate Arrangements:}

After April 1982, when the peg to the French franc was discontinued, the exchange rate was managed with reference to a basket of ten currencies. However, by end-1991, the authorities ceased the practice of adjusting the exchange rate to offset inflation differentials with key trading partners. Since May 9, 1994, the Malagasy franc has been determined through an open outcry auction system. The exchange rate in terms of the SDR at end-January 2004 was FMG 9,978.94 = SDR 1 .

Madagascar accepted the obligations under Article VIII, Sections 2(a), 3, and 4 on September 18, 1996 and maintains no restrictions on the making of payments and transfers for current international transactions.

\section{Last Article IV Consultation:}

Madagascar's consultation cycle is governed by the decision approved by the Executive Board on July 15, 2002. This decision stipulates that, subject to certain exceptions, an Article IV consultation with a member receiving financial assistance under a Fund arrangement is expected to be completed within 24 months of the conclusion of the previous Article IV consultation. The 2002 Article IV consultation discussions with Madagascar were held during October 7-12 in Antananarivo and during November 11-13 at headquarters. The staff report was discussed by the Executive Board on December 23, 2002.

\section{Technical Assistance:}

Department Dates

FAD July-August 1994

FAD September 1995

FAD
September 1996

\section{Purposes}

Assess the decline in the country's tax effort and recommend measures to mobilize additional revenue.

Assess the need for reforming the vahue-added $\operatorname{tax}$ (VAT) and develop a strategy to improve tax and customs administration.

FAD advisor to assist the authorities in implementing the recommendations of FAD September 1995 mission. Assignment

\section{Results of Missions}

Elimination of all ad hoc tax exemptions, beginning with the 1995 budget, recommended.
Broadening of the VAT base and the creation of a special office for the monitoring of transactions in the free export zone recommended.

The large-taxpayer unit established. Implementation of VAT reform continued. 
extended to March 1998.

\begin{tabular}{|c|c|c|}
\hline Department & Dates & Purposes \\
\hline FAD & December 1997 & $\begin{array}{l}\text { Review existing tax } \\
\text { incentives and prepare } \\
\text { action plan to improve the } \\
\text { performance of the tax and } \\
\text { customs departments. }\end{array}$ \\
\hline $\mathrm{FAD}$ & February 1998 & $\begin{array}{l}\text { Hold follow-up discussion } \\
\text { on tax and tax administra- } \\
\text { tion teform program. }\end{array}$ \\
\hline FAD & March 1998 & $\begin{array}{l}\text { Examnine and assess } \\
\text { budgetary expenditures, } \\
\text { control, and management. }\end{array}$ \\
\hline
\end{tabular}

FAD

April 1998

June 1998

FAD

June 1998

FAD

FAD
May, September, and December 1998 and April, May, and June 1999

July 1998

\section{Results of Misstions}

Program proposed to curtail exemptions and implement an exemption-monitoring scheme.

Schedule for the preparation and implementation of key measures established.

Comprehensive report prepared for the reform of budget planning, execution procedures, and control systems.

Agenda for follow-up mission identified (July 1998). be addressed in the area of customs administration.

Conduct follow-up visit on expenditure control.

Hold follow-up discussions on tax and tax administration reform.

Identify measures to strengthen customs administration.
Action plan, based on the report of the FAD mission of March 1998, established.

Assistance for the revision of the VAT law provided, and a VAT reimbursement system introduced for free export zone producers.

Report prepared on duty assessment and collection procedures, the coordination between the preshipment inspection company and the customs department, the computerization strategy, and organizational issues. 


\section{Department}

FAD

$\begin{array}{ll}\text { FAD } & \\ & \text { June } 1999 \\ \text { FAD } & \text { February-March } \\ & 2001\end{array}$

FAD January-February

FAD

MFD

MFD

MFD 2003

Dates

January 1999

2001

July-August 2003

August 1993-99

April-May 1994

May 1995

\section{Purposes}

Help implement earlier FAD recommendations on improving public expenditure management.

\section{Results of Missions}

Aide-mémoire prepared on progress in harmonization of budget and treasury classifications, computerization of treasury offices, implementation of a cash management and financial plan, and implementation of a budget and accounting classification for the decentralized budget.

Strengthen tax audit system of large-taxpayer unit.

Examine and assess budgetary control and management.

Examine and assess tax and customs administration.

Examine and assess tax policy and assist in tax policy design.

Assign a bank supervision advisor and assist in the adoption of an interbank foreign exchange market.

Review the modalities for liberalizing the exchange regime and accompanying monetary and credit measures.

Review interbank foreign exchange market, develop indirect instruments of monetary management, and reduce banks' excess reserves.
Action program developed and agreed with the authorities.

Comprehensive report prepared for the reform of budget planning, execution procedures, and control systems.

Comprehensive action plan and report on establishment of an oversight committee prepared.

Comprehensive action plan and report on next steps prepared.

Supervisory agency strengthened.

Interbank exchange market adopted in May 1994. Central bank base rate and reserve requirements increased.

Recommendations adopted during the second half of 1995. 


\begin{tabular}{|c|c|c|c|}
\hline Department & Dates & Purposes & Results of Missions \\
\hline MFD & $\begin{array}{l}\text { October-November } \\
1998\end{array}$ & $\begin{array}{l}\text { Assess the operation of the } \\
\text { foreign exchange and } \\
\text { treasury bill markets; } \\
\text { introduce the euro } \\
\text { quotation currency. }\end{array}$ & $\begin{array}{l}\text { Recommendations for treasury } \\
\text { bill market adopted in } \\
\text { November } 1998 .\end{array}$ \\
\hline MFD & December 1998 & $\begin{array}{l}\text { Prepare the change to the } \\
\text { euro as pivot currency. }\end{array}$ & Euro introduced as scheduled. \\
\hline MFD & May-June 1999 & $\begin{array}{l}\text { Prepare for introduction of } \\
\text { continuous-quotation } \\
\text { foreign exchange market, } \\
\text { including procedures } \\
\text { manual and market rules. }\end{array}$ & $\begin{array}{l}\text { To be implemented by end- } \\
2003 \text {. }\end{array}$ \\
\hline MFD & $\begin{array}{l}\text { October-November } \\
1999\end{array}$ & $\begin{array}{l}\text { Assess exchange } \\
\text { regulations and compliance } \\
\text { with the Basel Core } \\
\text { Principles for effective } \\
\text { banking supervision. }\end{array}$ & $\begin{array}{l}\text { Recommendations on banking } \\
\text { regulation to be adopted. }\end{array}$ \\
\hline MFD & $\begin{array}{l}\text { November- } \\
\text { December } 2002\end{array}$ & $\begin{array}{l}\text { Assess progress in the } \\
\text { implementation of the Core } \\
\text { Principles for effective } \\
\text { banking supervision; and } \\
\text { examine the early warning } \\
\text { system for detecting bank } \\
\text { failures. }\end{array}$ & $\begin{array}{l}\text { Recommendations being } \\
\text { implemented. }\end{array}$ \\
\hline MFD & November 2002 & $\begin{array}{l}\text { Take an inventory of } \\
\text { microfinance institutions } \\
\text { and define a strategy for } \\
\text { their supervision. }\end{array}$ & $\begin{array}{l}\text { Recommendations being } \\
\text { implemented. }\end{array}$ \\
\hline MFD & November 2002 & $\begin{array}{l}\text { Assess management of } \\
\text { foreign reserves and } \\
\text { propose concrete steps for } \\
\text { its improvement. }\end{array}$ & $\begin{array}{l}\text { Recommedations being } \\
\text { implemented. }\end{array}$ \\
\hline $\mathrm{MFD}$ & March 2003 & $\begin{array}{l}\text { Review central bank } \\
\text { internal audit functions. }\end{array}$ & Awaiting final report. \\
\hline
\end{tabular}




$\begin{array}{llll}\text { MFD } & \text { March 2003 } & \begin{array}{l}\text { Review microfinance } \\ \text { regulation. }\end{array} & \text { Awaiting final Teport. } \\ \text { Department } & \text { Dates } & \text { Purposes } & \text { Results of Missions } \\ \text { MFD } & \text { June 2003 } & \text { Review currency reform } & \begin{array}{l}\text { Recommendations being } \\ \text { implemented }\end{array} \\ \text { STA } & \text { October 1995 } & \begin{array}{l}\text { Review money and banking } \\ \text { statistics. }\end{array} & \begin{array}{l}\text { Recommendations for data } \\ \text { improvements adopted. }\end{array} \\ \text { STA } & \text { June 2001 } & \begin{array}{l}\text { Review balance of } \\ \text { payments compilation } \\ \text { system. }\end{array} & \begin{array}{l}\text { Recommendations for } \\ \text { improvemcnts accepted and } \\ \text { being adopted. }\end{array} \\ \text { STA } & \text { July-August 2001 } & \begin{array}{l}\text { Review money and banking } \\ \text { statistics. }\end{array} & \begin{array}{l}\text { Recommendations for data } \\ \text { improvements accepted and } \\ \text { being adopted. }\end{array}\end{array}$

\section{Resident Representative:}

Madagascar has had a Fund Resident Representative since September 1989. Mr. Samir Jahjah took up the post of Resident Representative at end-September 2003. 


\section{Madagascar: Relations with the World Bank}

(As of November 2003)

\section{Partnership in Madagascar's postcrisis recovery}

1. With the resolution of the political crisis in July 2002, the World Bank was in a position to accelerate its assistance because suspension of projects had been avoided during the crisis. As an immediate response to the crisis, the World Bank prepared an Interim Country Assistance Strategy (I-CAS), centered the (i) restructuring of the project portfolio to better align project with postcrisis recovery needs; (ii) preparation of an Emergency Economic Recovery Credit (EERC) in the amount of US $\$ 50$ million; and (iii) preparation of a Rural Transport Project in the amount of US\$80 million. The Bank's interim objective is to limit and reverse the impact of the crisis on the poor by supporting the government's reconstruction and recovery program, with particular emphasis on (i) supporting a safety net for the poor; (ii) assisting the private sector to restarting production; and (iii) ensuring a minimum of public service delivery.

2. The political crisis ended in July 2002 , but the social and economic impact was extremely pronounced, especially on a country with a GNP per capita of US\$260 (in 2000), one of the poorest in the world even before the crisis had commenced. The direct negative impact on national income for 2002 was estimated at half a billion dollars. The total economic cost is much larger, taking into account the loss of confidence, forgone direct investments, forgone exports, and physical destruction.

3. On July 26,2002 , the new government presented its reconstruction and development program to the Friends of Madagascar donors' conference, during which donors pledged approximately US $\$ 2.3$ billion over a period of four years. A second successful Friends of Madagascar donors conference took place in October 2003, at which the government and donors took stock of developments over the year and discussed the government's priorities of governance, transport, and education. Donors reaffirmed their support for the implementation of the government's poverty reduction strategy paper (PRSP) and the Education-for-All initiative.

\section{Bank Group strategy and lending operations}

4. The Bank's portfolio implementation during the crisis greatly suffered because (i) counterpart funds were not available (which is not a new phenomenon but worsened during the crisis); (ii) procurement of certain goods and works was stopped due to problems within the relevant government agencies; (iii) decision ${ }^{-m a k i n g}$ authority was unclear; (iv) sector ministries were unable to function; and (v) project activities could not be carried out in certain areas of the country. During the crisis, the implementation performance of 16 projects out of 19 was rated unsatisfactory. However, since the end of the crisis, almost all projects have readjusted their work plans and have witnessed accelerated implementation. 
Disbursements of Bank investments dramatically increased from \$37.3 million in fiscal-year (FY) 02 to $\$ 164.5$ million in FY 03.

5. In response to the end of the political crisis, the World Bank prepared an I-CAS, which was presented to the Board of Executive Directors in November 2002. The I-CAS's objective is to assist the government to mitigate the impact of the crisis on the poor by supporting selected areas of the government's program. Bank instruments used in response to the crisis include portfolio restructuring (including a measure that waives the provision of nontax counterpart funds for selected projects until the end of 2003), new lending (including an emergency credit), and policy advice. The mix of these instruments is determined by (i) the Bank's objective of limiting the impact on the poor; and (ii) the activity of other partner organizations in Madagascar.

6. The Bank is supporting the new government through analytical work and policy dialogue in several areas. Most of the studies had aiready commenced before the crisis and were reorientated to meet urgent information needs, for example on poverty developments, the economic cost of the crisis and priorities for reconstruction, and short-term policy to strengthen communities. In addition to the ongoing work on poverty monitoring, the Bank has completed analytical studies and reports on rural development and poverty reduction, decentralization, measures to revive the export processing zone (EPZ), tourism, a country procurement review (CPPR), and a Country Financial Accountability Analysis (CFAA).

7. The Bank's current portfolio consists of 15 projects, with total commitments of around US\$717.2 million, of which about US\$281 million was disbursed as of August 5, 2003. Of total commitments, around 45 percent are in the social sectors, 33 percent in infrastructure, transport, energy, and mining, 16 percent in rural development, and 6 percent in finance and private sector development.

8. The Bank has prepared its Country Assistance Strategy (CAS) for FY 04-06, which centers on supporting the government's recently developed PRSP and helping Madagascar's efforts to reach the Millennium Development Goals. Key upcoming analytical and advisory assistance foreseen over the next CAS period includes a public expenditure review and a development policy review. Proposed new investments include Transport Infrastructure, Governance, Environment, an Integrated Growth Poles Project, and a Watershed Project. The Bank also plans on moving to programmatic lending during this CAS period through Poverty Reduction Strategy Credits (PRSCs) targeting education, nutrition, and bealth.

\section{IMF-World Bank collaboration in specific areas}

9. IMF and World Bank staff maintain a close working relationship, especially with respect to (i) completion of the full PRSP; (ii) reforms set out at the decision point of the Initiative for Heavily Indebted Poor Countries; (iii) analyses and reforms in public financial management; (iv) other governance reforms, including customs; (v) support of the privatization program; and (vi) participation in the Integrated Framework trade project. The table below includes a short description of each of the areas and specific support provided by the two institutions with respect to policy advice. 
Bank/Fund Collaboration

Other govemance reforms

Privatization program
Area

Completion of full PRSP
Description

Together with multiand bilateral donors, Bank/Fund work provides contimuous technical assistance toward completion of full PRSP, planned in 2003

Regular joint Bank/Fund supervision missions; joint preparation of HUPC "Tracking PovertyRelated Speading" assessment and action plan

Public financial management
Integrated framework/ trade anaiytical work.
Specialized Advice/ Reforms Supported by Fund

Macroeconomic framework: growth, revenue, expenditure, and trade projections

Reforms linked to budgetary accounting and controls

\section{Tax analysis and reform, strengthening of control organs; expenditure management}

Customs

New government has embarked on heavy governance reform agenda; Bank/Fund staff works closely with UNDP/EU/AfDB staffs in assisting development of implementation plans

Close joint monitoring of government's large privatization program, comprising four major and several dozen smaller public enterprises

Integrated Framework (IF) paper FY 04

\begin{abstract}
All sectors, especially monitoring of privatization receipts of large enterprises (petroleum, telecommunications)
\end{abstract}

Macro projections for IF framework paper
Specialized Advicd Reforms Supported by Bank

Poverty analysis; rural poverty study; and education and health sector work

Reforms in education, health, rural transport sector
Expenditure monitoring and analysis, especialiy in sectors important for poverty recuction

Anticorruption agenda, decentralization, and judicial sector reform

\begin{abstract}
All sectors. Reforms in petroleum, telecommunications and air transport sector are linked to ongoing adjustment program.
\end{abstract}

Sectoral analysis for IF paper 
10. Areas where the Fund takes the lead role relate to policy advice and reforms with respect to (i) overall economic policy advice and macroeconomic targets; (ii) tax policy and administration; (iii) budgetary accounting; (iv) treasury procedures; (v) public sector wage policy; and (vi) monetary management and exchange rate policy. The Bank team actively participates in discussions between the Fund and the government in all these areas, especially, however, with respect to the setting of overall macroeconomic targets, as well as tax policy. At the request of the authorities, a tax policy technical assistance mission from the Fiscal Affairs Department of the IMF was fielded during July-August 2003.

11. Areas in which the World Bank takes the lead are related to specific sector advice in the areas in which the Bank has active lending operations (especially in the social sectors, infrastructure, agriculture and environment) as well as a number of analytical studies described in paragraph 6.

12. Joint responsibility for policy advice from the Bank and the Fund concern (i) budgetary procedures, including expenditure execution; (ii) the functioning of internal and external budget control institutions; (iii) reform of customs; (iv) trade policy; and (v) monitoring and continuation of the privatization program. Furthermore, the two institutions jointly support the PRSP process, the HIPC Initiative process, and the completion of the Integrated Framework trade initiative (see Table 2).

13. Questions may be referred to Mr. Willem van Eeghen (473-2399), Country Program Coordinator for Madagascar at the World Bank. 


\section{Madagascar \\ Operations Portfolio (IBRD/LDA and Grants)}

(as of 08/05/2003)

\section{IERD/LA *}

Total Disbursed (Active)

271.5

of which has been repaid

0.00

Total Disbursed (Closed)

$1,676.36$

of which has been repaid $\quad 201.29$

Total Disbursed (Active + Closed)

of which has been repald

$1,907,485,785.59$

$201,294,774.24$

Total Undisbursed (Aclive)

445.68

Total Undlsbursed (Closed)

Total Undisbursed (Active + Closed?

445.68

\section{Active Prolacts}

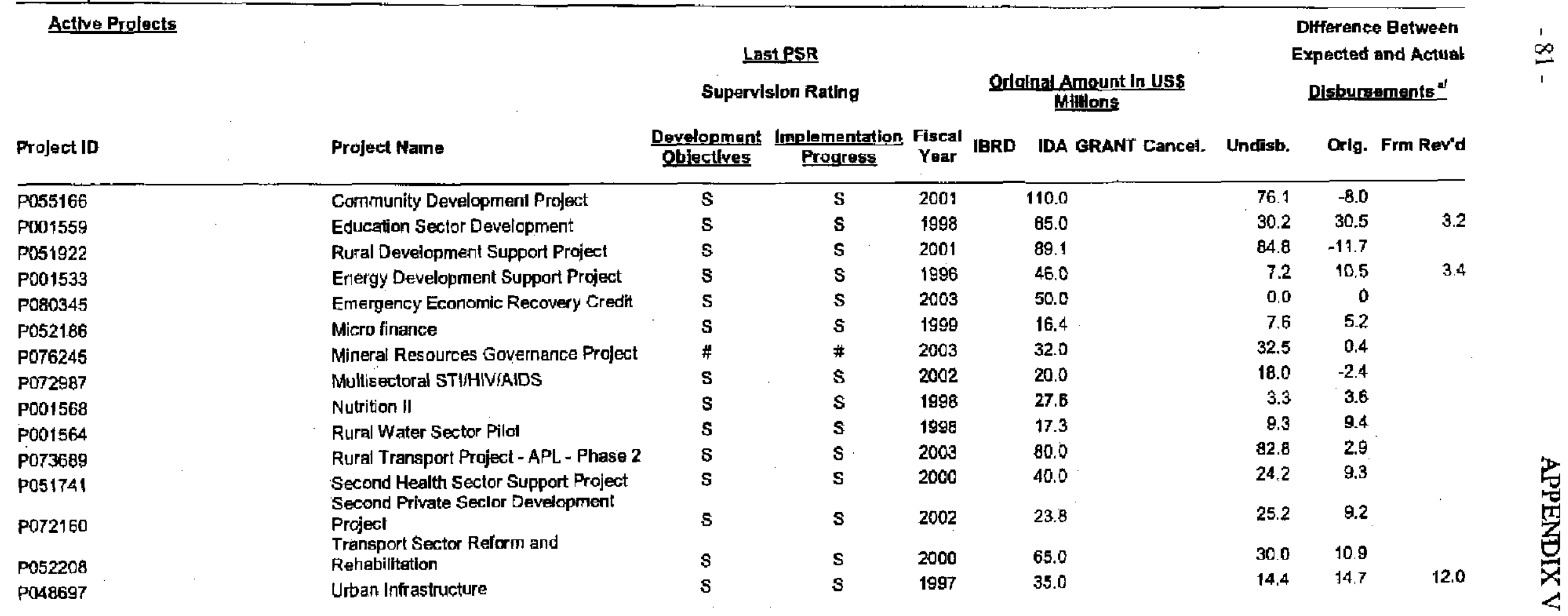

CInternational Monetary Fund. Not for Redistribution 


\section{Madagascar: Statistical Issues}

1. Madagascar's database remains weak, particularly the real sector, government finances, the balance of payments, and social indicators. The authorities are aware of these deficiencies and are working, with technical assistance from the international community, including the Fund, to strengthen their statistical system. Madagascar is taking steps to participate in the General Data Dissemination System (GDDS). To this end a GDDS coordinator has been nominated, and metadata are under preparation. STA balance of payments and monetary and financial statistics technical assistance missions visited Antananarivo in June and July 2001, respectively. Following a request by the authorities, a multisector statistics mission will visit February 12-25, 2004.

\section{Real sector}

2. The introduction in $\mathbf{1 9 9 6}$ of a system of regular business surveys has contributed somewhat to an improvement in the compilation of national accounts data. However, data remain weak and incomplete, particularly for the agricultural sector. Data on the use of resources are aiso inadequate, and data for changes in inventories are not reported. Shortterm indicators of economic activity are not generally available. Significantly, in January 2002 , the publication of a quarterly index of industrial production by export processing zone (EPZ) enterprises was introduced, with external technical and funancial assistance. There are plans to begin developing a quarterly industrial production index (covering non-EPZ enterprises), should discussions concerning United Nations Development Program (UNDP) financial assistance be completed early.

3. A revamped consumer price index (CPI), with weights based on the1993 household survey and covering four principal cities, was introduced in July 2000. The CPI is generally reported to Fund staff on a timely basis; however, during the period January-August 2002, the compilation of the price index was distupted and reporting delayed; timely reporting resumed in September. Data on producer prices and nationwide employment are not available.

\section{Government finance}

4. The latest government finance statistics (GFS) data reported to STA for publication in the GFS Yearbook are for 2001 and cover the consolidated central government. However, many classification problems remain (requiring extensive use of adjustment entries to current expenditure), and detailed information is missing in the time series since 1996 for budgetary and nonbudgetary breakdowns and for central government debt. Madagascar does not report subannual data for publication in International Finance Statistics. 


\section{Balance of payments}

5. Since 1984, the Central Bank of Madagascar (BCM) has been in charge of compiling and disseminating balance of payments statistics, and in 1997 it implemented the fifth edition of the Balance of Payments Manual (BPM5). However, the current compilation system is flawed in many respects: the external trade data are derived from customs data, which are affected by inadequate coverage at the level of recording procedures, and by large amounts of smuggling, particularly in the mining sector. Moreover, the customs processing system has experienced numerous technical disruptions since 1998. As a result, the external trade data have limited serviceability and require many manual corrections. The current implementation of the ASYCUDA (automated system for customs data, Version 2.7) was largely completed by mid-2002, with the system installed in most customs offices. There are plans to upgrade the customs processing system to ASYCUDA +

6. The balance of payments statistics mission (June 5-20, 2001), which reviewed the compilation system within the framework of the BPMS, left with the authorities a number of recommendations aimed at improving the existing compilation system, including institutional reforms within the BCM and measures to improve interagency cooperation. In November 2002, the authorities indicated that the committee in charge of finalizing external trade statistics had been reactivated and was contributing to improved data collection and analysis. In 2002, significant progress was also made on the implementation of banking sector surveys, aimed at ensuring comprehensive coverage of service transactions in the balance of payments. In April 2003, the authorities indicated that the reporting forms for bank settlements and respondents' surveys had been improved through new questionnaires on foreign direct investment for nonfinancial corporations, and that further work was being undertaken to review the classification of all reported transactions in a format consistent with BPMS.

7. The compilation of external debt statistics is generally satisfactory, and the authorities are benefiting from technical assistance by the United Nations Conference on Trade and Development (UNCTAD) in installing the latest version of the Debt Management and Financial Analysis System (DMFAS). This system is not yet fully operational; some data entry remains to be completed, and certain DMFAS modules remain to be installed by UNCTAD technicians.

\section{Monetary statistics}

8. A STA monetary and financial statistics mission visited Antananarivo in July 2001 to follow up on the recommendations of the 1995 mission, assist the staff of the BCM in addressing data collection, compilation, and dissemination issues, initiate work toward introduction of the methodology in the Monetary and Financial Statistics Manual, and assist the authorities in drafting GDDS metadata for the financial sector. The 2001 mission found that the BCM implemented most of the recommendations of the monetary and financial statistics mission of 1995, including the reclassification of some accounts of the monetary 
authorities and commercial banks and the revision of valuation procedures for foreigncurrency-denominated accounts. The 2001 monetary and financial statistics mission specifically recommended (i) the widening of statistical coverage to microfinance institutions; (ii) the inclusion of the Savings Bank and Postal Administration in the "Other Depository Corporations" subsector; and (iii) the improvement in the timeliness of the BCM's balance sheet. An action plan for the implementation of the mission's recommendations has been agreed with the authorities. Beginning in August 2001, monetary data for publication in International Financial Statistics (IFS) have been reported to STA regularly by e-mail. During the 2002 political crisis, the timeliness of monetary data reporting was uneven; however, the authorities resumed regular reporting starting at midyear. Data for the monetary authorities and deposit money banks through October 2003 have been published in the IFS. 
Madagascar: Core Statistical Indicators

(As of January 5,2004 )

\begin{tabular}{|c|c|c|c|c|c|c|c|c|c|c|c|c|}
\hline & $\begin{array}{c}\text { Exchange } \\
\text { Rates }\end{array}$ & $\begin{array}{l}\text { Interna- } \\
\text { tional } \\
\text { Reserves }\end{array}$ & $\begin{array}{l}\text { Central } \\
\text { Bank } \\
\text { Balance } \\
\text { Sheet }\end{array}$ & $\begin{array}{c}\text { Reserve' } \\
\text { Base } \\
\text { Money }\end{array}$ & $\begin{array}{l}\text { Broad } \\
\text { Money }\end{array}$ & $\begin{array}{c}\text { Interest } \\
\text { Rates }\end{array}$ & $\begin{array}{l}\text { Consumer } \\
\text { Price } \\
\text { Index }\end{array}$ & $\begin{array}{l}\text { Exports' } \\
\text { Imparts }\end{array}$ & $\begin{array}{c}\text { Current } \\
\text { Account } \\
\text { Balance }\end{array}$ & $\begin{array}{c}\text { Overall } \\
\text { Govern- } \\
\text { ment } \\
\text { Balance }\end{array}$ & $\begin{array}{l}\text { GDPI } \\
\text { GNP }\end{array}$ & $\begin{array}{c}\text { Extemal } \\
\text { Debt/Debt } \\
\text { Service }\end{array}$ \\
\hline $\begin{array}{l}\text { Date of latest } \\
\text { observation }\end{array}$ & $12 / 19 / 03$ & $10 / 31 / 03$ & $10 / 31 / 03$ & $10 / 31 / 03$ & $10 / 31 / 03$ & $11 / 21 / 03$ & $10 / 2003$ & $\mathrm{Q} / 03$ & $\mathrm{Q} 2 / 03$ & $9 / 2003$ & 2002 & $9 / 2003$ \\
\hline Date received & $11 / 19 / 03$ & $11 / 21 / 03$ & $11 / 21 / 03$ & $11 / 21 / 03$ & $11 / 21 / 03$ & $11 / 25 / 03$ & $11 / 25 / 03$ & $10 / 15 / 03$ & $10 / 15 / 03$ & $11 / 15 / 03$ & $3 / 10 / 03$ & $10 / 15 / 03$ \\
\hline $\begin{array}{l}\text { Frequency } \\
\text { of data }\end{array}$ & $\mathrm{D}$ & $\mathrm{M}$ & $M$ & $M$ & $M$ & M & M & $Q$ & $Q$ & M & A & A \\
\hline $\begin{array}{l}\text { Frequency of } \\
\text { reporting }\end{array}$ & $\mathrm{W}$ & $\mathrm{M}$ & $\mathbf{M}$ & M & $M$ & M & $\mathrm{M}$ & $Q$ & $\mathrm{Q}$ & M & $\mathrm{V}$ & $A$ \\
\hline Source of update & A & A & A & $\mathrm{A}$ & A & A & A & A & A & A & A & A \\
\hline $\begin{array}{l}\text { Mode of } \\
\text { Ieporting }\end{array}$ & $\mathrm{M}$ & $M$ & $M$ & $M$ & $M$ & M & $\mathrm{M}$ & M & M & $\mathbf{M}$ & $\mathrm{v}$ & $\mathrm{V}$ \\
\hline Confidentiality & $\mathrm{C}$ & C & $\mathrm{C}$ & $\mathrm{C}$ & $\mathrm{C}$ & C & $\mathrm{C}$ & C & C & $\mathrm{C}$ & $\mathrm{C}$ & $\mathrm{C}$ \\
\hline
\end{tabular}

Notes: Frequency of data: $D=$ daily; $M=$ monthly, $Q=Q$ uarterly, $A=$ annual.

Frequency of reporting: $W=$ =weekly; $M=$ monthly; $Q=$ quarterly; $A=$ annually; $V=i$ iregularly in conjunction with staff visits.

Source of update: $A=$ direct reporting by the Central Bank of Madagascar, Ministry of Finance and Economics, or other official agency.

Mode of reporting: $M=$ mail (including e-mail); $V=$ staff visits.

Confidentiality: $\mathrm{C}=$ untestricted use 


\title{
INTERNATIONAL MONETARY FUND
}

\section{REPUBLIC OF MADAGASCAR}

\author{
Fourth Review Under the Poverty Reduction and Growth Facility and \\ Requests for Extension of the Arrangement and Additional Interim Assistance Under \\ the Enhanced Initiative for Heavily Indebted Poor Countries- \\ Supplement on Request for Increased Access Under the \\ Poverty Reduction and Growth Facility \\ Prepared by the African Department \\ (In consultation with the Policy Development and Review Department) \\ Approved by A. Basu and Martin J. Fetherston
}

March 15, 2004

1. Madagascar was hit by Cyclone Gafilo two times between March 7 and March 10, 2004, after the staff report for the fourth review of the Poverty Reduction and Growth Facility (PRGF) arrangement had been issued. ${ }^{1}$ First official estimates are that 149 people are feared dead.

2. According to the Malagasy authorities, the cyclone devastated the Sava region, center of the vanilla production, as well as two ports in northern Madagascar through which vanilla is exported. In 2003, vanilla exports amounted to SDR 154 million, or 27 percent of Madagascar's total exports. Vanilla exports in 2004 were originally projected to amount to SDR 116 million, reflecting a 25 percent decline over 2003 , due to theft and insecurity in the producing areas. Preliminary estimates by staff, in the aftermath of Cyclone Gafilo, are that vanilla exports would decline further to SDR 40 million in 2004 (see table below).

\begin{tabular}{|c|c|c|c|c|c|c|c|}
\hline \multicolumn{8}{|c|}{$\begin{array}{l}\text { Madagascar: Export of Goods, 2003-04 } \\
\text { (In milljons of SDRs, unless otherwise indicated; cumulative since the beginning of } t\end{array}$} \\
\hline & \multirow[t]{3}{*}{2003} & \multicolumn{6}{|c|}{2004} \\
\hline & & \multicolumn{2}{|c|}{$\mathrm{Q1}$} & \multicolumn{2}{|c|}{ Q2 } & \multicolumn{2}{|c|}{ Total } \\
\hline & & $\overline{\text { Proj. }}$ & $\overline{\text { Ptel. }}$ & Proj. & $\overline{\text { Rev. }}$ & Proj. & Rev. \\
\hline Exports of goods & 566.0 & 176.2 & 137.0 & 325.0 & 266.8 & 613.5 & 537.4 \\
\hline Of which : exports of vanilla & 153.8 & 59.6 & 20.4 & 88.5 & 30.3 & 115.8 & 39.7 \\
\hline \multicolumn{8}{|l|}{ Mernorandum item: } \\
\hline Exports of vanilla (in percent of total exports) & 27.2 & 33.8 & 14.9 & 27.2 & 11.4 & 18.9 & 7.4 \\
\hline
\end{tabular}


3. The cyclone damaged the Andapa region, which is the rice production center of the northern part of Madagascar. It is also likely that shellfish exports will be affected, as most of the aquacultural infrastructure in Mahajanga was destroyed.

4. The dislocation of people and the physical damage caused by the cyclone have resulted in social and economic costs of very large, but not yet fully quantified, proportions for Madagascar. The program review is thus proceeding amid unusual uncertainty with respect to the macroeconomic context. The revised balance of payments takes into account a preliminary assessment of the impact of the cyclone on only the vanilla industry and the corresponding foreign assistance.

5. The authorities have appealed for assistance from the international community, and Cyprus, the European Union, France, Libya, and the United States have already announced their intention to help. The World Bank staff is also considering various options to provide support.

6. In the attached supplementary letter of intent (LOI) to the Acting Managing Director, dated March 15, 2004, (Appendix I), the Malagasy authorities request an angmentation of access under the current PRGF arrangement from the equivalent of SDR 79.43 million ( 65 percent of quota) to the equivalent of SDR 91.65 million (75 percent of quota). The additional access of the equivalent of SDR 12.22 million (10 percent of quota) would constitute immediate balance of payments support linked to an expected increase in the trade deficit associated with the cyclone. Thus, the originally scheduled amount of SDR 11.347 million will be increased by the amount of the augmentation, upon approval of the fourth review of the PRGF arrangement (see revised Table 1 attached).

7. A revised proposed decision is attached, which provides for the augmentation of the PRGF arrangement and the increased amount of the fifth disbursement associated with this review (Decision A). The proposed decision on Interim HIPC assistance has also been updated to reflect the schedule of repayments due following the Board meeting on Madagascar (Decision B).

\section{Staff APrRaisal}

8. Based on the expected impact of Cyclone Gafilo on Madagascar's balance of payments, and the commitment by the authorities to achieving their macroeconomic objectives, the staff supports the authorities' request for an augmentation of access under the current PRGF arrangement in an amount equivalent to 10 percent of quota. When more precise estimates of the impact of the cyclone on growth, public finances, and the balance of payments become available, staff will make an assessment of the additional donor support that would be desirable.

9. The staff welcomes the support atready announced by the international conmunity and hopes that further relief will be provided, preferably in the form of grants. 
Ms. Anne O. Knueger

The Acting Managing Director

International Monetary Fund

Washington, D.C.

March 15, 2004

Dear Ms. Krueger,

This letter is intended to serve as a supplement to the Memorandum of Economic and Financial Policies (MEFP) signed on March 2, 2004.

Cyclone Gafilo, described as the most violent in 50 years, hit Madagascar on March 7 and again on March 10, 2004, and has destroyed important agricultural production infrastructure, including rice and vanilla, and the aquaculture in the northem part of the country. Vanilla and shellfish are important exports of Madagascar, and these developments are bound to significantly reduce exports in 2004. The Government of Madagascar requests that, in view of the immediate balance of payments need arising from the cyclone destruction, the amount of the current Poverty Reduction and Growth Facility (PRGF) arrangement be increased from the equivalent of SDR 79.43 million (65 percent of quota) to the equivalent of SDR $91.65^{\circ}$ million ( 75 percent of quota). We request that the additional amount of SDR 12.22 million (10 percent of quota) be disbursed in full, together with the fifth originally scheduled disbursement to the equivalent of SDR 11.347 million, following the completion of the fourth review under the arrangement.

The Government of Madagascar would like to reiterate its commitment to achieving the macroeconomic objectives and structural reforms contained in the MEFP referred to above.

Sincerely yours,

$/ \mathrm{s} /$

Benjamin Andriamparany Radavidson

Minister of Economy, Finance, and the Budget

Antananarivo, Madagascar
$/ \mathrm{s} /$

Gaston Ravelojaona

Governor

Central Bank of Madagascar

Antananarivo, Madagascar 
Table 1. Madagascar: Tentative Work Program Under the Proposed Revised PRGF Arrangement, 2003-05

\begin{tabular}{|c|c|c|}
\hline Date & Action & Disbursement \\
\hline October 2003 & $\begin{array}{l}\text { Mission to conduct fourth review under the } \\
\text { PRGF arrangement and discussion of } \\
\text { program for } 2004 \text {. }\end{array}$ & \\
\hline March 2004 & $\begin{array}{l}\text { Executive Board consideration of the } \\
\text { fourth review under the PRGF } \\
\text { arrangement, including increased access of } \\
\text { the equivalent of SDR } 12.22 \text { million. }\end{array}$ & SDR 23.567 million \\
\hline May 2004 & $\begin{array}{l}\text { Mission to conduct the fifth review under } \\
\text { the PRGF arrangement. }\end{array}$ & \\
\hline July 2004 & $\begin{array}{l}\text { Executive Board consideration of the fifth } \\
\text { review under the PRGF arrangement. }\end{array}$ & SDR 11.347 million \\
\hline November 2004 & $\begin{array}{l}\text { Mission to conduct the sixth review under } \\
\text { the PRGF arrangement and discussions for } \\
\text { Article IV consultation. }\end{array}$ & \\
\hline February 2005 & $\begin{array}{l}\text { Executive Board consideration of sixth } \\
\text { review under PRGF arrangement, and } \\
\text { conclusion of the Article IV consuitation. } \\
\text { Arrangement expires. }\end{array}$ & SDR 11.347 million \\
\hline
\end{tabular}


Table 2 Madjogacur: Hziance of Payments, 2000-08

(in millions of SORs, thless otherwise indicaud)

\begin{tabular}{|c|c|c|c|c|c|c|c|c|c|c|c|c|}
\hline & \multirow[t]{2}{*}{2000} & \multirow[t]{2}{*}{2001} & \multicolumn{2}{|c|}{20002} & \multicolumn{2}{|c|}{20005} & \multicolumn{2}{|c|}{2004} & \multirow[t]{2}{*}{2005} & \multirow{2}{*}{\multicolumn{2}{|c|}{$\begin{array}{ll}2006 & 2007 \\
\text { Projections }\end{array}$}} & \multirow[t]{2}{*}{2008} \\
\hline & & & Prog. & Pret. & Prop. & Propj. & Prog. & Rev. & & & & \\
\hline 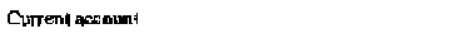 & .165 .4 & +6.4 & -1550 & .203 .5 & -168.6 & -193.4 & -230.3 & -242.5 & -143.2 & .262 .6 & -276.3 & .297 .7 \\
\hline Goods end services & -214.8 & -114.3 & .202 .4 & -224.8 & -275.9 & -369.4 & -360.4 & -437.1 & -3712 & -394.1 & -409.8 & -130.6 \\
\hline Trade batance & .78 .6 & $: 1.6$ & -913 & -90.5 & -90.1 & -154.9 & -225.1 & .301 .3 & -216.9 & -214.9 & -195.3 & .1727 \\
\hline Exports & G2B.S & 757.9 & 4060 & 375.0 & 584.9 & 566.0 & 613.5 & 537.4 & 757.6 & 881.1 & $1, \theta \mid 4.6$ & $1,115.5$ \\
\hline Imports & -707.1 & -746.3 & 4973 & A65.5 & -675.0 & .30 .9 & -838.6 & -818.6 & +9745 & $-1,096.0$ & $-1,109.8$ & $-1,218.2$ \\
\hline Net serpices (nell & -136.2 & -325.9 & -111.1 & -134.3 & +185.8 & -154.3 & -135.2 & . 35.8 & .154 .4 & -179.2 & -724.5 & -257.9 \\
\hline Servicas, recelpss & 279.9 & 276.3 & $\mid \$ 1.2$ & 373.1 & 247.3 & 235.7 & 287,8 & 287.3 & 315.4 & 346.0 & 3519 & 377.8 \\
\hline Services, payments & -407.1 & -492.2 & .292 .3 & .397 .4 & -413.1 & -3902 & -423.1 & +23.1 & .469 .8 & -525.2 & .518 .5 & -635.7 \\
\hline Incotne (met) & -53.3 & -46.7 & -585 & +53.0 & $-56 . d$ & -57.9 & -47.9 & 47.9 & -38.0 & -43.9 & -51. & -63.0 \\
\hline Rectipts & 16.6 & 187 & 15.5 & 20.1 & 193 & 1.9 & 15.7 & 15.7 & 20.0 & 21.2 & 225 & 23.6 \\
\hline Peymmis & -69.9 & -65.4 & -74.0 & -73.1 & -75.7 & $\$ 5.8$ & -53.6 & -63.6 & -58.0 & -65]$. & $-74,4$ & -86.6 \\
\hline Of whigh : government truerest" & -39.6 & 41.4 & -43.9 & -43.1 & $-42,5$ & -40.1 & .42 .4 & -42.4 & -29.9 & -29.0 & $-27,9$ & $-27,0$ \\
\hline Cuerent mansfors & 102.6 & 114.6 & 105.9 & 747 & 16.3 .6 & 173.9 & 177.9 & 242.4 & 166.0 & 179.4 & 185.3 & 195.8 \\
\hline Governmenl & 25.5 & 24.9 & 44.9 & 6.2 & 98.3 & 99.3 & 92.9 & 157.4 & 75.9 & 799 & 84. & 88.6 \\
\hline Budget aid & 16.5 & 36.7 & 513 & 22.1 & $\mathbf{9 8 . 8}$ & 98.9 & 82.5 & 147.0 & 66.4 & 30.4 & 74.6 & 79.1 \\
\hline HIPC relief ' & 0.0 & 1 B.8 & .20 .2 & 29.2 & 22 & 21.1 & 19.9 & 19.9 & 0.0 & 0.0 & 0.0 & 0.0 \\
\hline Grants & 18.5 & 179 & 31.3 & 1.9 & 66.7 & 66.7 & 62.6 & 62.6 & 66.4 & 70.4 & 74.6 & 79.9 \\
\hline 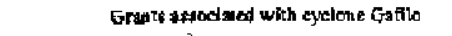 & & & & & & & & 64.5 & & & & \\
\hline Othex (net) ${ }^{3}$ & 5.0 & -11.8 & -6.4 & -15.9 & 9.5 & 10.4 & 10.4 & 10.4 & 9.5 & 9.5 & 9.5 & 9.5 \\
\hline Private & $\pi .1$ & 89.7 & 61.0 & 68.0 & 65.3 & 74.6 & 85.0 & 85.0 & 90.1 & 95.9 & 101.7 & 107.3 \\
\hline Cappital and finarcial aceoum & 71.6 & 65.9 & 67.3 & 104.1 & 1687 & 166,9 & 288.2 & 28.8 .2 & 303.5 & 314.9 & 327.8 & 344.6 \\
\hline Capital eceounl \{goveramenl projecl prants\} & 87.0 & 88.6 & 40.4 & 44.7 & 63.5 & 63.8 & 124.5 & 124.5 & 132.0 & 139.9 & 148.3 & 157.2 \\
\hline Financial acenomi & 34.0 & 90.9 & 27.1 & 25.5 & 1047 & 33.9 & 163.7 & 163.7 & 173.5 & 175.0 & 179.5 & 157.4 \\
\hline Direst inveatmen! & 52.9 & 73.1 & 5.0 & 6.4 & 13.1 & 9.1 & 52.0 & 52.0 & 60.6 & 60.6 & 60.6 & 60.6 \\
\hline of which : privatization receipls & 51 & 10.9 & 3.0 & 33 & 3.7 & 0.0 & 0.0 & 0.0 & 0.0 & 0.0 & 0.0 & 0.0 \\
\hline Other & -189 & 17.8 & 22.1 & 19.1 & 91.1 & 24.8 & - III.T & 111.7 & 112.9 & 1144 & $\cdot 118.9$ & 126.8 \\
\hline Governunerl & 6.1 & 21.5 & 34.6 & 48.1 & 96.6 & 49.8 & 187.0 & $1 / 70$ & 117.3 & 319.4 & 136.5 & 1343 \\
\hline Drawing & 86.9 & 90.2 & 126.7 & 120.3 & 167.6 & 120.8 & 186.1 & 186.1 & 164.2 & 172.9 & 183.3 & 194.5 \\
\hline Projeel drawings & 46.2 & 60.4 & $97 . d$ & 54.5 & 100.0 & 83.5 & 107.5 & 107.5 & 1362 & 141.3 & 151.7 & 162.9 \\
\hline Budgetary Apport & 39.3 & 26.8 & 79.3 & 6.5 .7 & 56,6 & 37.3 & 78.6 & 78.6 & 28.0 & 31.6 & 31.6 & 31.6 \\
\hline Non-gavernment & 1.4 & 30 & 0.0 & 0.0 & 6.0 & 0.0 & 0.0 & 0.0 & 0.0 & 0.0 & 0.0 & 0.0 \\
\hline Amertizutian ' & -80.8 & -69.7 & -72.1 & .72 .2 & -710 & -71.0 & -69.1 & -89.1 & -46.8 & -53.5 & -56.8 & +60.2 \\
\hline Privake soctor anarization & $a .0$ & .7 .5 & .7 .5 & -7.9 & -5.4 & -7.7 & .3 .3 & -53 & 4.4 & .5 .0 & -7.6 & -7.4 \\
\hline Banks, net & -25.8 & 3.8 & -25.0 & $-21,1$ & 0.0 & +13.3 & 0.0 & $D .0$ & D. & 0.0 & 0.0 & 0.0 \\
\hline Dther (inci. enrows and omiesions) ${ }^{1}$ & -49.4 & -1136 & 0.0 & 33.9 & 0.0 & 69.2 & 0.0 & 0.0 & D., 0 & 0.0 & 0.0 & 0.0 \\
\hline Gverall balance & .93 .8 & 19.6 & $\triangle 87.5$ & .59 .4 & -0.4 & -26.5 & 57.9 & 45.7 & 62.3 & 52.3 & 51.5 & 46.9 \\
\hline Finnacing & 93.8 & -19.6 & 87.5 & $\$ 9.4$ & 0.4 & 26.5 & .579 & -45.7 & -67.3 & .52 .3 & $-51 . .5$ & -46.9 \\
\hline Nex fortion asseb (mereare -) & $2 B .4$ & -76.9 & 30,0 & 40,0 & -40.1 & .14 .0 & -46.7 & $-84,5$ & $=117.6$ & -109.7 & -108.8 & -105.1 \\
\hline Unea of Fund credit (nei) & 14.2 & 21.4 & B.6 & 8.6 & 17.3 & 5.9 & 17.3 & 29.5 & 3.2 & -16.9 & -17.6 & -17.6 \\
\hline Distursemento & 38,0 & 22.7 & 12.4 & 11.4 & 22.7 & 15.3 & 22.7 & 34.9 & 113 & 0.0 & 0.0 & 0.0 \\
\hline Reprymants & -3.8 & .1 .3 & -2.7 & -2.7 & -5.4 & .5 .4 & .5 .4 & $-3-4$ & $\$ .1$ & -16.9 & -37.6 & -17.6 \\
\hline Other assets, net (inereabe -) & .5 .7 & .98 .3 & 21.4 & 314 & .57 .4 & -20.0 & .114 .0 & .114 .0 & -120.8 & -92.9 & -91.2 & 87.6 \\
\hline Nel change in arreas (exchudinz central bank) ${ }^{3}$ & 5.8 & 1.6 & 0.0 & 1.5 & -9.5 & -4.5 & 0.0 & 0.0 & 0.0 & 0.0 & 0.0 & 0.0 \\
\hline Debt relief and eanceliation ${ }^{6 T}$ & 59.6 & 53.8 & 57.5 & 57.9 & 50.0 & 50.0 & I8.E & 38.8 & 553 & 575 & 57,3 & $\$ 8.2$ \\
\hline \multicolumn{13}{|l|}{ Mumaromdum itans: } \\
\hline Grenls (in percent of GDP) & 3.8 & 3.2 & 2.5 & 15 & 4.4 & 4.7 & 5.1 & 6.7 & 4.6 & 45 & 4.4 & 4.4 \\
\hline Lasus [in percent of (ODP) & 0.2 & 0.6 & 1.6 & 1.4 & 2.6 & 1.3 & 2.8 & 2.8 & 2.6 & 2.4 & 2.4 & 2.4 \\
\hline Dirent irvealment (in percent of GDP? & 1.8 & 2.1 & 0.1 & 0.2 & 0.4 & 6.2 & 1.2 & 1.2 & 13 & 1.2 & 1.2 & 1.1 \\
\hline Curtent aceoust \{in perefrs of GDP\} & & & & & & & & & & & & \\
\hline Excluding net ofti wial transiers & -6.5 & -2.0 & .5 .8 & -6.1 & -9.3 & .7 .5 & .7 .6 & -9.5 & -7.0 & -7.0 & -6.9 & -6.9 \\
\hline Inowding nel offieial trank fars & -5.6 & -1.3 & $\$ .5$ & -5.9 & -4.6 & -4.9 & -5.4 & -3.7 & $-5,4$ & $-5,4$ & -53 & -5.3 \\
\hline Gouss official reserves & 218.7 & 217.5 & 296.9 & 266.6 & 324.0 & 286.6 & 4001.6 & 425.0 & $\$ 11.3$ & 614.2 & 705.4 & 793.0 \\
\hline 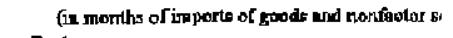 & 2.4 & 31 & $\$ .9$ & 4.1 & 3.5 & 3.1 & 3.8 & 4.0 & 4.3 & 4.5 & 4.7 & 49 \\
\hline Exchsigge ralos & & & & & & & & & & & & \\
\hline Malapay francesSDR (period average) & $8,951.0$ & B,791.0 & $8,895.0$ & 8,7743 & $\cdots$ & $\cdots$ & $\cdots$ & $\ldots$ & $\cdots$ & $\ldots$ & $\cdots$ & -. \\
\hline Malngary fancer/U.S. dollar (praind average) & $6,787.2$ & 6.591 .5 & 6,BBO.1 & 6,5923 & $\ldots$ & $\ldots$ & $\ldots$ & $\cdots$ & $\cdots$ & $\ldots$ & $\ldots$ & $\cdots$ \\
\hline
\end{tabular}

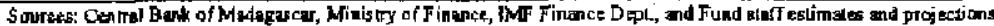

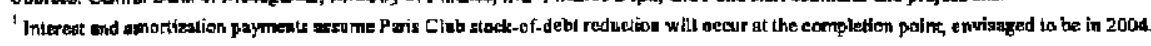

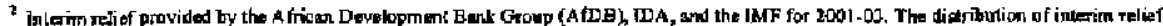

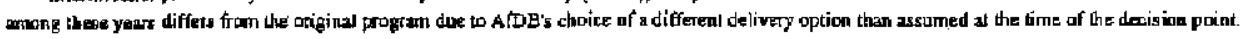

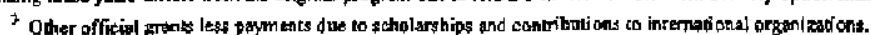

4 Includes commarcial oredis recrived or graniad.

${ }^{5}$ Froen 2000 on, inciudes exiernal commercial antears.

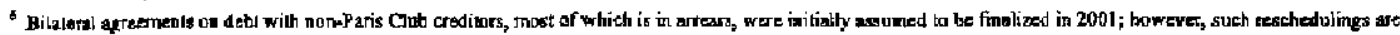

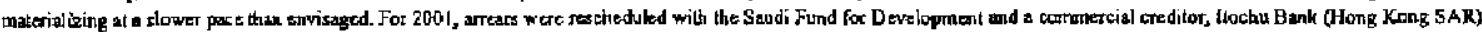

7 Assuming a $P$ arip Clut flow rexchedoling an Cologne terms agreed in 2001, of which a part is atributable to FisC relied

I En percent of export of goods and nomfactor services.

${ }^{9}$ Th percent of goremarient reventre. 


\section{Statement by the IMF Staff Representative \\ March 17, 2004}

1. The following supplementary information has become available since the issuance of the staff report. The thrust of the staff's assessment remains unchanged.

- Cyclone Gafilo, one of the most violent cyclones in 50 years, hit the country in March, adding to the damage already exacted by cyclone Elita in January and February 2004. In light of the additional balance of payments need, the authorities have requested an augmentation of access under the current PRGF arrangement, which the staff supports.

- The consumer price index declined by 0.8 percent from December 2002 to December 2003 , compared with the projection in the staff report of a 0.3 percent increase.

- Preliminary data indicate that tax revenue in 2003 was 10 percent of GDP, consistent with the projection in the staff report.

- With the economy emerging from the 2002 political crisis, imports during JanuaryAugust 2003 almost recovered to the level during the same period in 2001. For the rest of the year, imports rose sharply as the tax and tariff relief law took effect on September 1. As a result, the 2003 imports surpassed the 2001 level by 10 percent.

- $\quad$ From end-December 2003 to end-February 2004, the Malagasy franc depreciated by 14 percent against both the euro and the dollar, reflecting rising imports and a weak export performance. The exchange rate stabilized somewhat in March, in part because of smoothing operations undertaken by the central bank.

- The international tender for the purchase of the cotton company, HASYMA, was launched on January 29,2004 , representing a step toward compliance with the endMarch 2004 structural performance criterion for the completion of the bidding process.

- The indicative targets for end-September 2003 were met, except for that on the domestic financing of the budget. 


\section{IMF Completes Fourth Review of Madagascar's PRGF-Supported Program, Approves an Augmentation of Access under the PRGF Arrangement, and a Disbursement of US\$34.7 Million}

The Executive Board of the International Monetary Fund (IMF) taday completed the fourth review of Madagascar's performance under an SDR 79.4 million (about US\$1 17.1 million) Poverty Reduction and Growth Facility (PRGF) arrangement.

Given the expected impact arising from cyclones that hit Madagascar in early March 2004, the Executive Board approved the Malagasy authorities" request to augment the access under the current PRGF of the equivalent of SDR 12.2 million (about US\$18.0 million). This additional amount is to be disbursed in full, together with the completion of the review.

The completion of the review and the additional amount will enable the release of SDR 23.6 million (about US\$34.7 milion), which would bring total disbursements under the program to SDR 69.0 million (about US\$101.6 million).

The Executive Board also approved the extension of the PRGF arrangement until March 1, 2005, with a rephasing of disbursements for July 2004 and February 2005 and an SDR 0.609 million (about US\$0.90 million) disbursement as additional interim assistance under the enhanced HIPC Initiative for Madagascar for the period March 19, 2004-June 30, 2004.

Madagascar's three-year program was approved on March 1, 2001 (see Press Release No. 01/7), and was extended on December 23, 2002 (see News Brief No. 02/133) until end-November 2004 from end-February 2004.

The PRGF is the TMF's concessional facility for low income countries. PRGF-supported programs are based on country-owned poverty reduction strategies adopted in a participatory process involving civil society and development partners and articulated in a Poverty Reduction Strategy Paper (PRSP). This is intended to ensure that PRGF-supported programs are consistent with a comprehensive framework for macroeconomic, structural, and social policies to foster growth and reduce poverty. PRGF loans carry an annual interest rate of 0.5 percent and are repayable over 10 years with a $5 \frac{1 / 2}{2}$-year period on principal payments. 
Following the Executive Board's discussion of Madagascar, Anne Krueger, Acting Managing Director and Acting Chair, stated:

"The Executive Board expressed its deep sympathy to the people of Madagascar for the dislocation and loss of life they have suffered from the recent cyclones. The cyclones are expected to have a significant adverse impact on the economy. However, the authorities remain committed to achieving their macroeconomic objectives and structural reforms with the assistance of the international community. The Fund is helping to cushion the balance of payments impact of the cyclones by augmenting Madagascar's access to Poverty Reduction and Growth Facility (PRGF) resources under the current program.

"Madagascar's economy rebounded in 2003 following the 2002 slump, and prospects for 2004 are for continued strong economic growth and low inflation. The implementation of the program supported by the Poverty Reduction and Growth Facility was satisfactory in the first half of 2003. However, fiscal slippages emerged in the second half of the year. The key challenges for the authorities in the period ahead will be to restore fiscal discipline, improve governance, strengthen the business climate to encourage private investment, and continue with structural reforms to boost exports and economic growth.

"The budget for 2004 allows for a modest growth in current expenditure, keeps the investment budget in line with the absorptive capacity of the economy and foreign financing, and aims at an ambitious tax revenue objective. To achieve these objectives, the authorities are taking measures to enhance expenditure control, remove administrative bottlenecks to reduce investment delays, improve the tax system, and expand the tax base. The authorities have appropriately decided not to renew the exemptions granted in the August 2003 tax and tariff relief law when they expire in September 2005, and to avoid such exemptions in the future.

"A key objective of the authorities' program is to improve the private sector's access to credit. The amendment of the Property Act in 2003 allows foreign investors to own land, which, combined with an improved land registry system, should facilitate the use of land as collateral for bank loans. The ongoing modernization of the judicial system is also essential to reduce banking risks.

"Following the expiry of the World Trade Organization's Agreement on Textiles and Clothing by end-2004, Madagascar may face increased competition in export markets. To meet these challenges, the authorities intend to persevere with prudent macroeconomic policies while accelerating structural reforms to improve the efficiency of the cotton sector, maintain low production costs, increase the output and quality of the vanilla crop, and diversify exports.

"The authorities are implementing a plan to privatize and rehabilitate the telecom, cotton, sugar, and utility companies in order to increase economic efficiency, private investment, and economic growth. Steadfast implementation of these reforns will contribute to raising incomes and reducing poverty in rural areas. 
"The Poverty Reduction Strategy Paper adopted in July 2003 provides a comprehensive and coherent framework for guiding the implementation of the Malagasy authorities" poverty reduction strategy. The authorities should now decisively implement the strategy and the remaining HIPC Initiative completion point triggers, so that Madagascar can benefit from additional debt relief as soon as possible," Ms. Krueger said. 


\section{Statement by Damian Ondo Mañe, Executive Director for Republic of Madagascar March 17, 2004}

Madagascar's economy is responding well to the comprehensive set of measures that were implemented in late 2002 and early 2003, following the political crisis of 2002. As Directors may recall this crisis brought about a major disruption in the economy, characterized by a very sharp drop in real GDP and aggravated the hardships of the population. To address this situation, the government established, with the assistance of the Fund, World Bank, other multilateral institutions and bilateral donors, a recovery and reconstruction plan. The measures taken were consistent with the PRGF program and aligned with the PRSP, and were aimed, among others, at stabilizing the economy, strengthening the business climate and governance, encouraging private sector investment, and diversifying the export base. An important component of the plan was also the provision of emergency assistance to the most vulnerable groups of the population. The recovery plan was successful in meeting most of the objectives that were set.

The Malagasy authorities have built on the progress made in 2003, and have strengthened their efforts in many areas, in particular in the fiscal sector where the authorities are implementing the FAD technical mission recommendations. As described in the authorities' Memorandum on Economic and Financial Policies for 2004, a wide range of measures consistent with the PRSP are being taken.

\section{Performance in $\mathbf{2 0 0 3}$}

Macroeconomic performance in 2003 continued to improve compared to the previous year, on the basis of the comprehensive measures undertaken. All the performance criteria for endJune 2003 were observed. Real GDP in 2003 is estimated to have increased by nearly 10 percent, compared with a program objective of 6 percent, with annual average inflation declining by 1.4 percent, compared to an increase of 8 percent in the program. Employment, in particular in the Export Processing Zone increased significantly, while activities in the other sectors of the economy, including the agricultural sector, were buoyant. Good progress was also made in improving access to education with the construction of additional classrooms, the recruitment of teachers, and the provision of basic supplies to primary school children. Access to basic health care was also expanded.

In the fiscal area, the authorities' objectives, for 2003, were: the improvement of government revenue to allow for increased social spending, and the promotion of economic recovery, while keeping the deficit at a level consistent with the program. In this regard, a wide range of measures were taken and included targeted revenue increases as well as reforms of tax and customs administration, and the strengthening of expenditure and treasury management. To help the economic recovery, following the major shock that occurred, the authorities reduced marginal income tax rates for individuals and companies, and the tariffs on a number of inputs for the agricultural and construction sectors. These measures were supplemented in mid-2003 by further temporary tax and tariff exemptions for the import of capital goods and selected commodities. The fiscal impact of these additional exemptions is 
estimated at about one quarter of one percent of GDP for 2003. On the expenditure side, there was an increase in extrabudgetary outlays in the area of education, security, public enterprise reform and additional domestic interest obligations, but overall expenditure was kept within program target. Government revenue, however, is estimated at 10.3 percent of GDP, instead of 10.6 percent, as programmed. As a result, the overall fiscal deficit ( on a commitment basis, excluding grants) was 7.9 percent of GDP. Although, this deficit improved compared to 2001 , it was higher than the program target of 7.6 percent of GDP.

The objective of monetary policy in 2003 was to reduce inflation while ensuring adequate scope for the expansion of credit to the economy, which is essential to the recovery. The stance of monetary policy was such that it helped to bring inflation under control. However, credit to the economy remained subdued in the first half of the year as businesses confidence took time to recover, and expanded in the second half of 2003 as credit to the government and crop financing picked up. The introduction of the new currency, the ariary which is equivalent to FMG 5, is proceeding smoothly

In the external sector, although there was broad improvement, the current account deficit target was higher than programmed, as exports increased by less than projected, as it is taking longer for the factories in the Export Processing Zone to return to full production. Import, in particular of capital goods, also increased significantly. The level of international reserves was lower than programmed. The authorities maintained their flexible exchange rate policy, and reflecting in large part the strengthening of the euro, the effective exchange rate depreciated in 2003.

The authorities implemented a number of structural reform measures in 2003. In particular, measures were introduced to enhance private sector confidence and development. The anticorruption commission was established. Steps were taken to strengthen tax and customs administrations and to combat fraud. The Property Act was amended to allow foreigners to own land. A comprehensive program of privatization was put in place, but met with delays due to technical problems and issues related to shareholder rights. The authorities remain committed to the privatization program and are working closely with the different partners to complete the process.

\section{Program for 2004}

The program for 2004 will continue the reform agenda. The authorities will implement policies that will ensure sustainable economic growth and the reduction in poverty, consistent with the PRSP. In this regard, the policies being implemented are expected to achieve real GDP growth of 6 percent, contain inflation at about 5 percent, and raise the level of international reserves to the equivalent of 3.8 months of imports. Efforts to improve the fiscal outlook and accelerate structural reforms will be intensified.

In the fiscal sector, while important progress has been made in restoring fiscal discipline and improving governance, this progress has to be consolidated and strengthened. Measures envisaged for 2004 go in this direction, and will focus, among others, on enhancing transparency and efficiency in public resource management, improving governance and 
raising the level of revenue. With the economy recovering, the authorities will focus more efforts on raising government revenue, and in this regard, the measures envisaged for 2004, are expected to raise the tax-to-GDP ratio from 10 percent in 2003 , to 11.2 percent in 2004 .

The authorities are taking steps that will continue the process of broadening the revenue base and maintaining the integrity of the VAT. The VAT is being extended to cover a number of previously exempted goods and services, and taxes on tobacco and cigarettes are being increased. The efforts to strengthen tax and customs administrations, as well as collection of tax arrears will be pursued. The authorities have also taken steps to simplify and streamline the tax system. In particular, the number of trade taxes and tariff rates are being reduced. These measures are also expected to contribute to enhance the openness of the economy and to reduce fraud. The Budget for 2004 includes measures aimed at reversing the increase in tax exemptions, and introduces a comprehensive overhaul of the tax system based on the recommendations of the Fund technical assistance mission.

On the expenditure side, the tight control over outlays will be maintained. However, the budget includes increases in poverty-related expenditure, as well as increases in capital expenditure, which is externally financed for the most part. The wage bill will be reduced to 5.1 percent of GDP in 2004, as no wage increase will be granted, although there is allowance for new recruitments in the areas of health, education, justice and security, and for the program of early retirement. The authorities also intend to clear all payments arrears outstanding at end-2003, and pay arrears of the SIRAMA and TELMA to sugar planters and creditors respectively. Procedures have been put in place to prevent the occurrence of new arrears. The authorities will also intensify their efforts to improve treasury accounting, simplify budget execution system, and strengthen the monitoring of budget implementation.

Monetary policy will be consistent with the inflation and growth objectives. In this regard, the authorities will monitor closely liquidity developments in the banking sector, and will also facilitate the development of a money market which should improve the effectiveness of monetary policy. The resumption of strong economic growth and the strengthening of the investment climate are expected to be conducive to demand for credit by the private sector. With the government not having recourse to net bank credit, the authorities have programmed broad money to increase by 12 percent during the year, which should be adequate to meet the needs of the economy while remaining consistent with the inflation objective. The availability of credit to small- and medium-sized enterprises is being improved through amendment to the Property Act to allow the use of property as collateral. The legal framework for microfinance activities is being finalized and operations are expected soon. Although the banks are well capitalized, nonperforming loans have been increasing, mainly as a consequence of the 2002 crisis. The authorities expect these nonperforming loans to decrease as the recovery takes hold, but they are, nevertheless, strengthening banking supervision and developing systems of early warning with assistance from the Fund. Moreover, the audit of reserves management and foreign exchange activities has begun, as recommended by the safeguards assessment report.

In the external sector, the prudent macroeconomic policies being implemented and the ongoing structural reforms will contribute to maintaining Madagascar's external 
competitiveness. The return of the EPZ factories to full production should also improve the export picture which, however, will be adversely affected by the drop in vanilla exports. On the import side, the public and private investment program and the lowering of a number of tariffs are expected to cause import to show a substantial increase, so that the external current account deficit(excluding transfers) is projected at about 7.6 percent of GDP. Reflecting a higher level of external financial assistance, the level of international reserves should increase to the equivalent of 3.8 months of imports. Morcover, in the context of its regional integration policy, and the launching of the customs unions of the COMESA, Madagascar is taking measures to harmonize its customs rules and procedures with those of the other countries of the COMESA.

As regards the debt profile, it remains broadly similar to that projected at the decision point. Madagascar has also finalized bilateral rescheduling agreements with most Paris Club and several non-Paris Club creditors. Contacts have also been made with all official creditors for the reconciliation of outstanding debt in preparation for reaching the completion point.

The comprehensive program of structural reforms will be pursued in 2004. In the fiscal sector, measures are being taken to further improve the operations of the tax and customs departments. In the public enterprise sector, the authorities are pursuing the efforts to complete the agenda of reforms for the several public enterprises for which reforms are underway.

\section{Cyclone Gafilo}

My authorities are fully committed to the reform process and to accelerate the reform agenda where possible. However, as has been the case in the past, the country remains subject to exogenous shocks, and especially to the vagaries of the weather. Last week Madagascar was hit twice by a violent hurricane which has caused large loss of lives and extensive damage to the agricultural sector and the infrastructure. The authorities are in the process of assessing the damages and the costs of reconstruction. They will consult with the staff, if any change needs to be made to the program's objectives, and will work with staff on the assessment of any additional support that may be required. However, in view of the extensive damage caused by the cyclone, as outlined in the staff supplement, and based on preliminary assessment, my Malagasy authorities are requesting an augmentation of access under the current PRGF arrangement in an amount equivalent to 10 percent of quota. I would, therefore, like to request Directors for their full support of the proposed decision, including the augmentation of access, to enable the Malagasy authorities to pursue their strong reform agenda. My authorities are thankful for the assistance that they have already received, and they hope that the international community will continue to stand by their side in these difficult times.

\section{Conclusion}

Despite the many challenges they faced, my Malagasy authorities are of the view that the overall performance of the economy improved significantly in 2003 , and that progress has been made in the reform agenda. The poverty-reduction program has been well implemented 
and is having a positive impact on the population. Under the 2004 program, the authorities' efforts will be intensified, as the macroeconomic situation has broadly stabilized. There is no doubt that the recent cyclone will have an impact on my authorities' efforts, but they remain fully committed to pursue steadfastly the reform agenda. They are thankful to the international community for the excellent assistance that has been provided to them, and which they are putting to good use. 